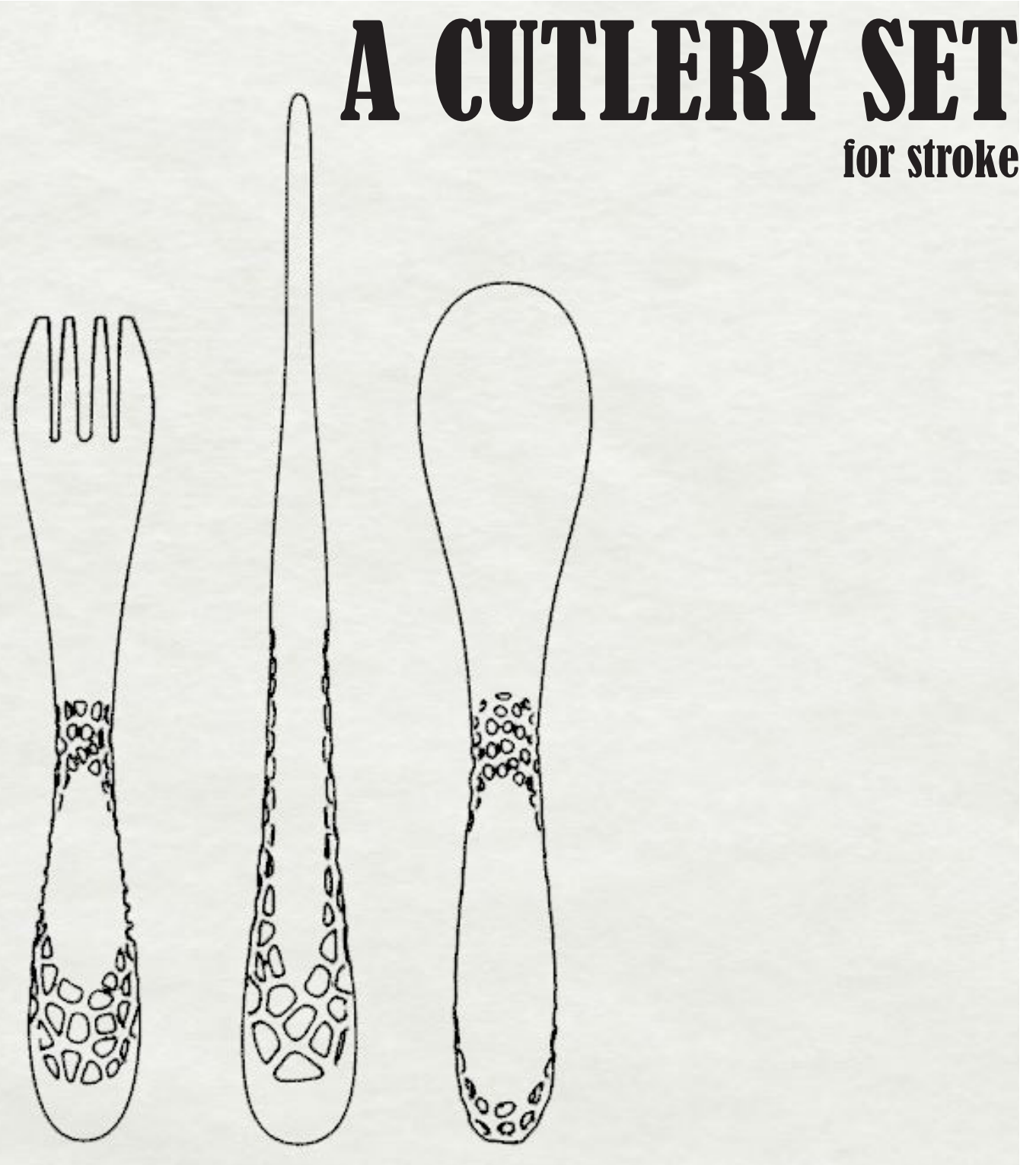





\title{
A cutlery set for stroke
}

By

\section{Lin Chen}

\begin{abstract}
A 90 point thesis submitted to the Victoria University of Wellington in partial fulfillment of the requirementsfor the degree of Master of Design Innovation in Industrial Design.
\end{abstract}

Victoria University of Wellington, School of Design 



\section{DEDICATIIN}

I dedicate this thesis to my family who have meant and continue to mean so much to me. Mum and Dad, I could not have done this without your help and support. Thank you for always trusting me and being with me when I need you.

Next, I dedicate this to my beloved grandparents. Although they are no longer in this world, their memories continue to regulate my life. I was not able to stay with you in the last minutes of life and attend your funerals because of my abroad study career. May you find peace and happiness in Paradise.

Last, I dedicate this to God Almighty, my creator. He has been the source of my strength and on His wings only have I soared. 



\section{ACKNDWLEDGEMENTS}

Dr Edgar Rodriguez-Ramirez, your continuing guidance and advice were highly valued throughout this journey.

Thanks to my fellow postgraduates, my good friends for pressing me on and keeping me motivated.

Thanks to the RAM Company for providing the significant technology support. I have used the proofreading service of Madeleine Collinge. Thanks to her academic help.

I would like to thank the fantastic staff of the School of Design, and other support faculties who have helped me throughout the whole thesis. 



\section{ABSTRACT}

Stroke is a common problem that affects approximately 700,000 patients annually in the United States alone and can cause long-term disabilities (Mallory, 2006, p.33). The long-term effects of a stroke can impact on the patient's ability to use one side of their body. Upper limb, lower limb, postural and communication difficulties are common factors that patients experience after a stroke (Perry, 2004), which can affect the patient's ability to eat (McLaren, 1997).

After a stroke, the impairment of an upper limb can lead to problems that make it difficult for people to use cutlery, and include poor grip, decreased muscle control, tremors and upper limb weakness (Brackenrige, 2016). Currently, there are some assistive technology (AT) cutlery sets aimed to help patients who have difficulty in eating and muscle control. Several reasons why stroke patients abandon AT cutlery are cost, appearance, and function (Vaes, 2014). However, an experiment (Torrens, 2013) in to adaptive cutlery products and previous research suggest that there is a large space to improve this, such as appearance and function.

AT cutlery sets have been criticized because of their appearance and inferior functions (Torrens, 2013). This then leads to a disconnection between the user and the AT cutlery products, in which the user experiences visible stigma and links the cutlery to an undesirable characteristic (Vaes, 2012). There are some limitations of the current AT cutlery sets that have been identified in the evaluation (Torren \& Smith, 2013), such as poor friction material, stereotypical shapes, and skin-tone handle colour. The limitations create an opportunity for the designers to improve the AT cutlery set to be a selected object and a pleasurable product to use in everyday life. 

The purpose of this research is to explore how human-centred design can reduce the stigma of using AT cutlery for stroke patients through addressing the appearance and interactions of spoons, forks and knives. The research involves the following steps: observing videos of stroke patients eating, interviewing clinicians, interviewing stroke patients, and iterative design with the supervision of clinicians.

Some of the main issues identified surrounding stroke patient eating and using cutlery include grip weakness, muscle contracture, and difficulty of flexion. Based on the product intervention model for stigma (PIMS) (Vaes, 2014), this study utilized existing research surrounding the evaluation of assistive technology. To help understand each stage of the user needs, previous feedback from health-care clinicians and patients will ensure the validity of ergonomic interventions and stigma strategy as a substitute for traditional cutlery design.

The output of this research includes a set of cutlery as well as assistive components. The design addresses a patient's difficulty in using cutlery through an adaptive ring to help the patients' grip. The utensils allow the user to either carry out eating activities at home or in a restaurant.

The feedback from occupational therapists and physiotherapists indicates that the cutlery should accommodate different levels of stroke severity. Based on the findings of literature reviews and feedback, I have focused my design on addressing the stroke patients' grip weakness, muscle contracture, and difficulty of hand flexion, whilst minimizing stigma, based on the PIMS strategies (Vaes, 2014). The design principle suggests that current cutlery designs with an integrated shape cannot meet all requirements of a stroke patient. Therefore, a combination of additional wearing components needs to be used to help dietary intake. 


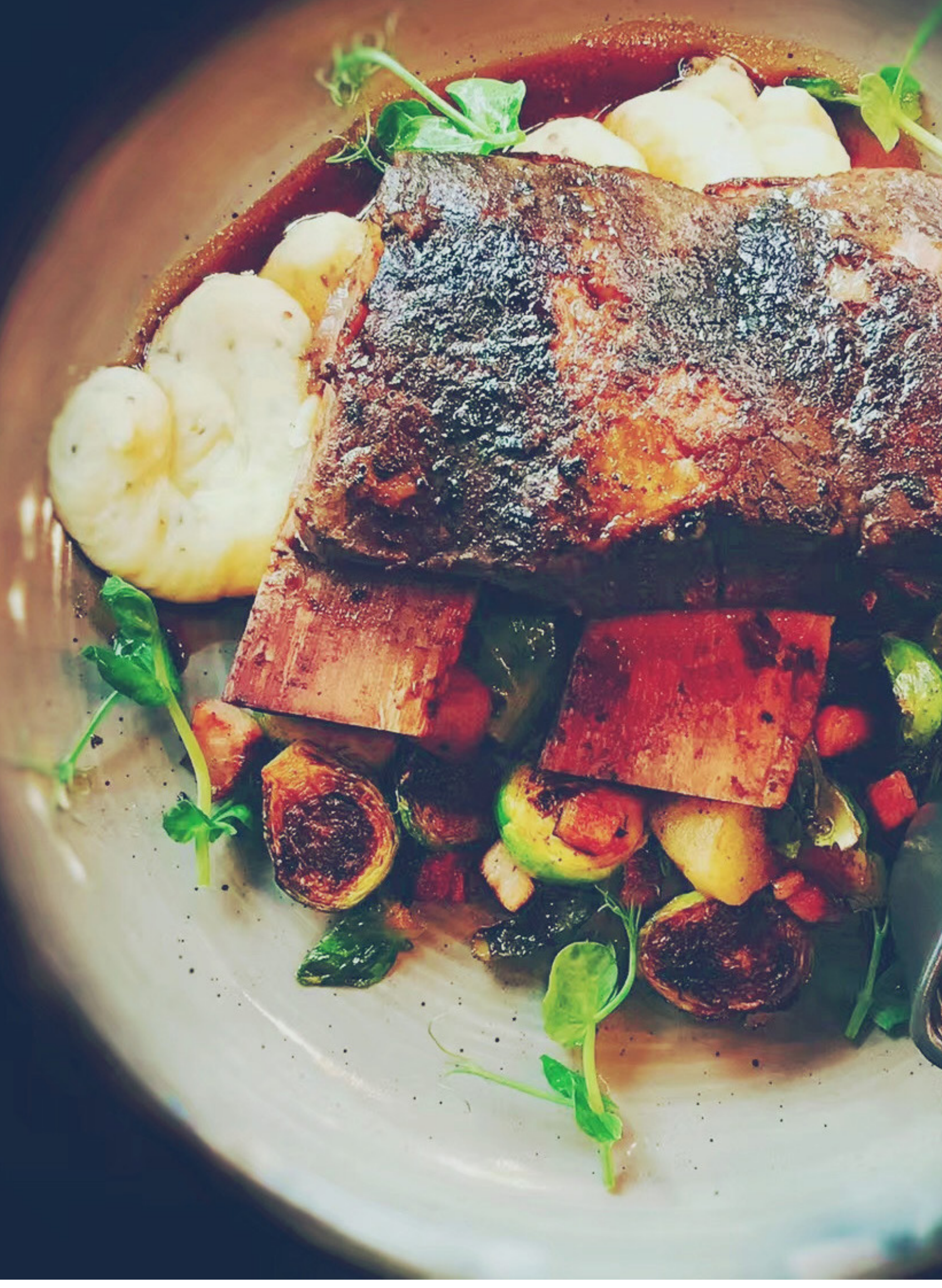




\section{TABLE DF CDNTENTS}

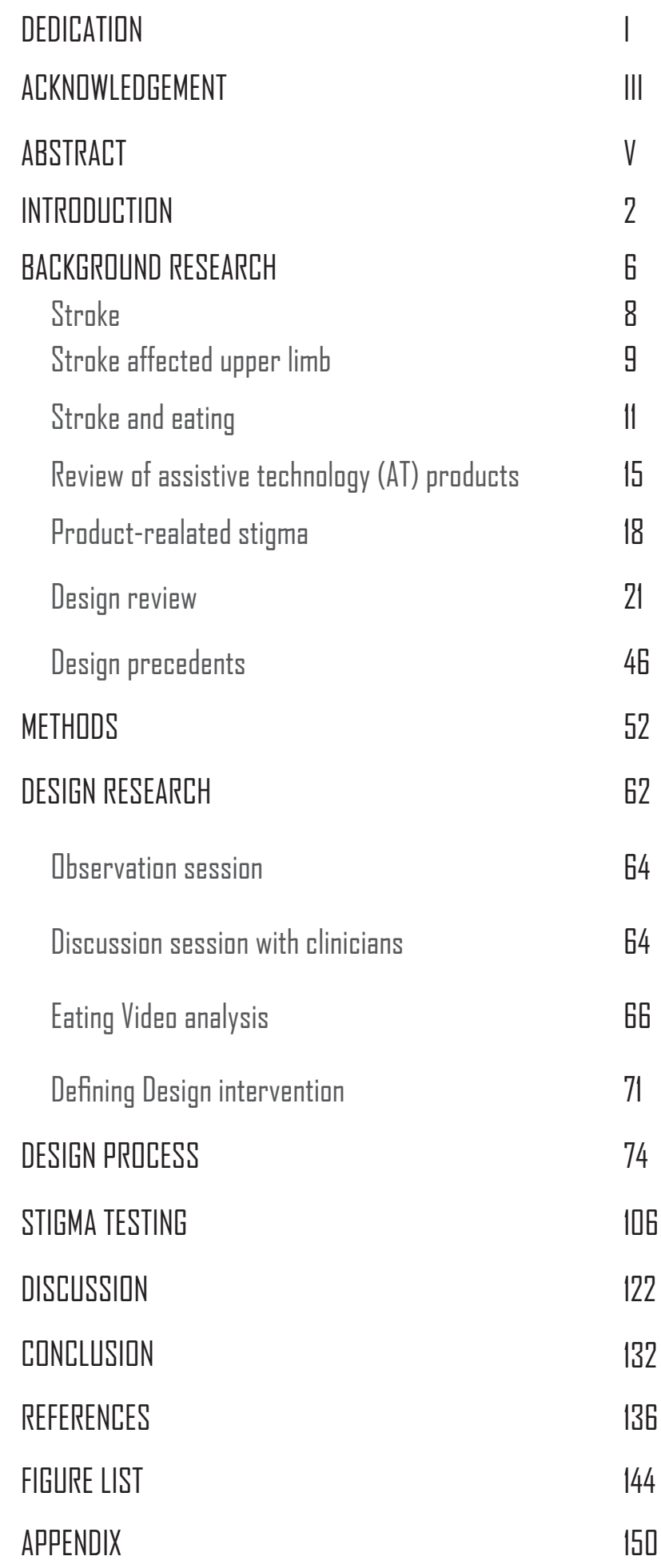


1 


\section{INTRODULTION}

With strokes accounting for six millions deaths annually, it is the leading cause of acquired physical impairment in adults in the world (Monteiro, n.d.). A stroke produces a wide range of impairments that affect physical ability, and the related impairments persist long beyond the acute phase or hospitalisation (Perry \& McLaren, 2002). Most common physical effects of stroke are muscle weakness, fatigue, pain, spasticity or flaccidity and shortening or lengthening of muscles (Stroke Association, 2013). The range of impairments also produces the impairments in upper limbs and affects the ability to eat. These inabilities include difficulty in maintaining an upright posture; loss of upper limb motor control/ sensation; and communication, visual, perceptual and attention deficits (Perry \& Mclaren, 2002). Recent studies (McLaren \& Dickerson, 2000) have shown that the increasing eating disability following an acute stroke exerts a negative impact in most stroke patients who have an affected upper limb. 
Goffman (1963, p.11) simply defines the term stigma "to refer to bodily signs designed to expose something unusual and bad about the moral status of the signifier". Stigma, and more specifically in people who have a disability, is part of the social, psychological and ethical context in which physical medicine and rehabilitation clinicians practice (Garland-Thomson, 2016, p. 997). In characterizing the stigma of products, the existing conception of stigma can increase by the emergency of product stigma (Jacobson, 2014). The stigma can be characterized as visible or invisible, controllable or uncontrollable, and as concerning appearance, behavior or group membership (Jacobson, 2014, p.77). The appearance of products is an important factor in the stigma experience and the communication alters as soon as an unfavorable product, linked to or in interaction with its user, becomes visible to bystanders (Vaes, 2014). In addition, some senses that can influence the stigma process relate to the smell, feel, sound and taste of the user or product, for example bad breath, the tactile feel of a prosthetic hand, or irritating product noises. In contrast, attractive products add value to the user experience and the user's daily life. Having the attractive features make the product appear trendy, stylish and desirable to most users, and stigma can gradually lessen, as their appearance is generally looked upon favourably (Skogsrød, n.d.). 
In recent years, Assistive Technology (AT) products comprise a wide range of implements and have been viewed as closer to medical tools rather than consumer goods (Skogsrød, n.d). Success of AT products relies on physical interactions and non-abandonment in long-term usage (McDonagh-Philp, Lebbon \& Torrens, 1999). Traditionally, in the areas of AT and design for disability, human factors must be taken into account during the design. Lacking of usability and poor performance are the main factors associated with 'displeased' products (Jordan, 1996). Besides usability, aesthetics were seen to be prioritized above almost all other considerations, even over functionality (Vaes, 2014, p. 26). For most AT product users in general, it is inconceivable not to have a choice in how they present themselves, yet it is a choice often denied or severely limited for users of assistive products (Skogsrød, n.d.). Especially different cultures lead a different table manner. A regular cutlery set in Western culture comprises forks, spoons, and knives. But the cutlery sets are different in others cultures. For instance, most Asian countries use chopsticks and spoon to eat.

People have eating difficulties caused by a stroke will rely on an adaptive cutlery set to eat. However, Torrens's study (2014) has shown a number of current AT cutlery sets lack consideration of aesthetics and functionality. Therefore, it shows an opportunity for the development of adaptive cutlery, as part of an AT device for assisting and accomplishing a user's eating tasks.

Initially, this research looks to combine a user-centred design approach and Vaes's (2014) stigma intervention in order to inform explorations of a new developed cutlery design. The research draws the connections between the user and designer by interviewing expert clinicians and by collecting the clinician's feedback in design iteration. . 



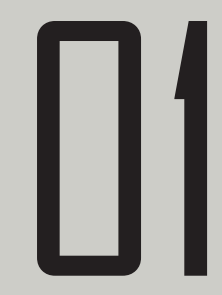

BACKCRDUND RESEARCH 


\section{Chapter summary}

The primary aims of this chapter are to examine previous studies and find evidence that suggests cutlery is an important device in the eating actions of stroke patients. The exploration of the background will inform the initial design research criteria and the usability assessment criteria. This chapter will be broken down into several themes so that the design criteria will be notified clearly and systematically. The comparison of the current market devices will be selected to evaluate the stigma aspects and it will guide the original design criteria that address the issues that have faced current users. 


\subsection{Stroke}

A stroke (also called cerebrovascular accident) occurs when a blood clot blocks artery (ischemic stroke) or a blood vessel breaks (hemorrhagic stroke), which interrupting blood flow to an area of the brain (Kirchhofs et al., 2009). How a stroke patient is affected, depends on where the stroke occurs in the brain, and how much the brain is damaged (National Stroke Association, 2014). Stroke, a common acute problem, has been estimated that around 1 in 4 men, 1 in 5 women aged over 45 years can expect to experience this if they live to 85 years old (Wolfe,2000). Some aspects of life after temper disorder and depression of life goals (Wade, 1994 \& Gall, 2001).

The Increasing eating disability following acute stroke exerts an adverse impact on consumpc of oral food and fluids. Eating disabilities have been demonstrated as obstructing dietary intake see during hospitalisation post-stroke (Axelsson et al., 1998; Davalos et al., c; Finestone et al., 1995; Gariballa et al., 1998; McLaren \& Dickerson, 2000). 


\subsection{Stroke affected upper limb}

A stroke produces a broad range of enduring impairments, and survivors' adaptation styles are influential features of life after a stroke (Perry \& McLaren, 2002). Most common physical effects of a stroke are muscle weakness, fatigue, pain, spasticity, flaccidity, and shortening or lengthening of muscles (Stroke Association, 2013).More than half of stroke survivors experience impairments of the upper limb in the chronic phase, including loss of strength, dexterity, spasticity, muscle contracture, pain and edema (Andriga, Port \& Meijer, 2013).

\section{Wrist and orthosis}

The most common effect of stroke patients on their wrist is muscle weakness, especially in the acute phase, but the spasticity can vary from light muscle flicker to severe spasm (Nijenhuis, 2014). The severe paresis has a higher risk of developing spasticity and muscle contracture of the wrist and finger flexor muscles. Spasticity occurs when the motor cortex has been damaged, as well as the part of the brain involved in the planning, control and execution of voluntary movements. Without an effective treatment and contracture prevention, patients are at the risk of developing a clenched fist: a hand which has deformed into a fist by shortening of flexor muscles of the fingers and soft tissues.
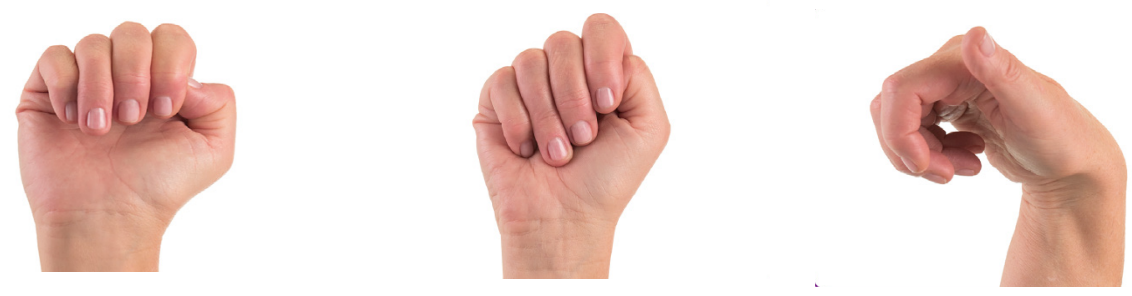

Figure 1.0 Affected hand (finger,thumb, wrist) (BOTOX, 2017) 
The most common noticeable wrist problem of stroke patients is muscle weakness, though not all patients have this problem (Nejienhuis, 2014). Due to personal factors, such as age, weight and life habits, the hand spasticity can vary from light muscle flicker to severe spasm. It can be a combination of hand contracture and weakness in patients, but others may have a paralyzed limb. All of this, however, depends on the rehabilitation treatment process that patients follow, which also relates to which recovery devices they use. Due to the shortage of arm movement and wrist limitation problems, the selected assistive devices need to be considered by rehabilitation progress and a clinician's consideration.

Treatment for the recovery of wrist function usually starts in the first four weeks after a stroke, and patients are expected to achieve prominent results in the first six months after the stroke (Timmermans, Seelen, Willmann, \& Kingma, 2009). Thus, every opportunity should be enhanced to regain motor ability in the affected upper limb. For those patients not able to return to motor capability in a short time, a further strategy should focus on achieving the goal of daily activities and maintaining movement of the arm. 


\section{Spasticity}

Spasticity is one of the common symptoms after stroke, which following a stroke occurs in about $30 \%$ of patients (Thibaut, 2013). Spasticity causes a motor disorder in post-stroke, which can give rise to a considerable variety of symptoms (e.g. clonus, dystonia, muscle weakness, abnormal reflex responses) (Thibaut, 2013). Spasticity and other positives signs of upper motoneuron syndrome could diminish a patient's ability to control their muscles during specific movements. Spasticity can also affect quality of life and be highly detrimental to daily function. Spasticity disturbs the intensity of the sensory input (e.g., the degree of stretching) and will give rise to an imbalance between inhibitory and excitatory fibres (Thibaut, 2013). The most frequent pattern of arm spasticity is internal rotation and adduction of the shoulder coupled with flexion at the elbow, wrist and fingers.

\subsection{Stroke and eating}

Stroke-related impairments not only produce physical disabilities, but also cognitive emotional and social consequences. Becker and Burry (Perry \& McLaren, 2002) identify three stages which aim at enabling stroke survivors to maintain their identity and self-worth whilst living in a changed relationship with the world. The three stages are as follows:

1. A period facing dysfunction and discontinuity.

2. Facing Transition.

3. Treatment and adaptation. 
The two emergent themes (Perry \& Mclaren, 2002), 'getting back to normal' and 'getting by', have reflected the attitude of early acute stroke effect and until the adaptive activities in social participation. The study (Perry \& McLaren, 2002) indicates that survivors have accepted stroke effects, but their social participation is minimal and their lives are not 'normal'. The dominance of table manners and food habits value independent management of meal items. However, shame resulting from mismanagement and inappropriate behaviour can cause discomfort and loss of appetite in fellow diners. Patients talked of shame at their appearance and the humiliation of eating dependency. With impairments precluding the use of traditional cutlery, food and eating were no longer experienced as enjoyable (Jacobsson et al., 2000).

The effect potentially impacts on all areas of life. Some aspects of life after stroke have been comprehensively investigated, such as function abilities in activities of daily living, mood disorder, motor abilities and depression (Wade, 1994; Gall, 2001). Other aspects, for instance the extent and impact of eating difficulties, are less well understood. Stroke survivors' perceptions and subjective experiences of eating have been investigated with work mainly focusing on dysphagia and in longterm care clinics (McLaren \& Dickerson, 2000). 
Stroke leads to many deficits that affect the ability to eat and increase the risk of nutrition loss. The relevant eating disabilities caused by a stroke include loss of upper limb motor control and sensation; problems chewing and swallowing; and communication, visual perceptual and attention deficits. The experiment results show that many stroke patients need help to eat. The clinical stroke observation of South London hospital (Perry, 2004) indicates that less than half (46.5\%) ate independently, and the rest of the stroke patients were judged to require supervision and assistance. Disabilities in eating resulting from these impairments were difficulties in seeing and perceiving items on a meal tray (vision, perception); in continuing to eat (attention); in selecting meals from a menu or expressing preference (communication); in cutting food, loading cutlery and inserting food in the mouth (arm movement); in maintaining an upright posture when eating; and in effecting lip closure, chewing or swallowing (oral preparatory, oral transport and pharyngeal stages of deglutition) (McLaren \& Dickerson, 2000, p.111). Significant evidence in the expert literature shows the prevalence of eating impairments in which posture and arm movements were affected most, affecting $84 \%$ of the 75 acute stroke patients (McLaren \& Dickerson, 2000). Observations of a selected group of patients eating at up to 14 days post-stroke revealed $57 \%$ were unable to manipulate cutlery and transfer food from plate to mouth, so arm movement impairment predicted reduced energy and protein intake (Jacobsson et al., 2000; McLaren \& Dickerson, 2000). 
As described in McLaren's (2000) assessment of effective eating tools, making sure a patient can eat independently is challenging for careers and family members. The stroke's pathological characteristics require skilled interventions and design approaches from a designer. Like jewelry design, it seeks to complement the body rather than attempt to be camouflaged against it (Pullin, 2009). In the evaluation of assistive technology products (AT) (Torrens \& Smith, 2013), the participant's perceptions indicate that AT cutlery do not satisfy people with disabilities. Many transcribed comments in the review of AT cutlery have been identified, such as the size of the handle, the tactile feeling of the handle, and the appearance (Torrens \& Smith, 2013, p.344). Through the comparison with AT cutlery, the conventional handle shape has been identified as an effective design principle for patients with neuro-muscular conditions (Torrens \& Smith, 2013), and the method of changing grip patterns and add high friction materials are not advisable. That experiment has demonstrated that the appearances of AT cutlery are not well received socially by the UK local society (Torren \& Smith, 2013, p. 346). 


\subsection{Review of assistive technology (AT) products}

For many stroke patients, independence in a particular situation (transferring, feeding, toileting, etc.) is a requisite ability and should be improved through the use of an assistive device. Currently, in the market, there are several cutlery sets and AT utensils which can facilitate stroke patients in their eating process.

Consequently, AT is treated as promising tools for self-management when there are significant barriers to stroke patients, families and professionals. The aim of AT products focuses on increasing, maintaining or improving functional capabilities of individuals with disabilities.

AT usually includes assistive, adaptive and rehabilitative devices for people with disabilities (Assistive Technology Act, 1998). The scope of AT products can refer to small items, large equipment or product systems. These products can be acquired commercially, modified or customized. Regarding people's disabilities, AT can range from low-tech (e.g., reachers, page turners, adapted spoons) to high tech (e.g., environmental control systems, computerized communication devices) (Stehle, 2016).

When considering the use of AT devices, the challenge is that the patient might decide to abandon the technology products. Cruz's study (2016) shows that the participants did not use assistive products for several reasons: not believing in their benefits, aesthetic issues, needing a more secure device, and other issues peculiar to each case. 
Phillips and Zhao (1993) identify four factors related to the abandonment of AT devices: (a) not considering the user's opinions, (b) ease of device procurement, (c) poor device performance and (d) changes in the user's priorities. They also suggested AT services could reduce the frequency of abandonment. Stehlo's study (2015) shows that combining the development of AT and consumer product design is not difficult, as long as designers focus simultaneously on functionality, user experience and profitability. However, AT product abandonment by users is still a concern for rehabilitation teams.

The primary aim of AT is developed with function in mind, whereas product design gives user experience and desire. But likewise, AT design can forego aesthetic and experiential concerns. A user-centred consumer design differs from the development process of AT in identifying the opportunity, validating the concept and commercializing the product. In terms of different quantity and quality demands, consumer goods focus on profitability and brand effect on the market. Stroke survivors, compared with the majority of customers of a market, are still a small group to be taken into account.

Although there is no doubt that developing assistive devices will lengthen the process, such as design process and manufacturing process, results should prove that it is a worthwhile investment. The assistive product should be more desirable to the target population as well as to consumer in general.

Universal or inclusive design has a long history of being associated with AT products. Inclusive design has brought the concept that not all users are able bodied within standard parameters of height, weight and cognitive abilities. Norman (2002) suggests AT products are in great need of inclusive design and applying a strategy for their appearances could potentially reduce stigma due to their becoming ordinary (Norman, 2002). 
For the development of assistive products, a combined method of relevant strategies from empathetic design and human-centred design could serve the designer well. In an effort to develop a more desirable AT that provides a more pleasurable user experience, my project focused on the emotional factor that is associated with using cutlery, and the emotional connection stroke survivors have with their current assistive technologies.

Ergonomic and usability

ISO 9241-11 (Brooke, 1996) suggests that measures of usability should cover:

- $\quad$ Effectiveness (the ability of users to complete tasks using the system, and the quality of the output of those tasks)

- $\quad$ Efficiency (the level of resources consumed in performing tasks)

- $\quad$ Satisfaction (users' subjective reactions to using the system)

However, the precise measures to be used within each of these categories of metric above can vary widely by an uncertain contextual environment. The System Usability Scale (SUS) is a simple scale giving a global view of subjective assessments of usability, and has been proved robust and reliable (Brooke, 1996). The SUS includes ten-items:

1. I think that I would like to use this system frequently.

2. I found the system unnecessarily complex.

3. I thought the system was easy to use.

4. I think that I would need the support of a technical person to be able to use this system.

5. I found the various functions in this system were well integrated.

6. I thought there was too much inconsistency in this system.

7. I would image that most people would learn to use this system very quickly. 
8. I found the system very cumbersome to use.

9. I felt very confident using the system.

10. I needed to learn a lot of things before I could get going with this system.

In combination with inclusive design principles, the SUS assessment can be revise and apply into the user testing.

\subsection{Product-realated stigma}

The experience of stigma is common among human beings (Crandall, 2000). Everyone has experienced stigmatization at some points in their lives, be it a feeling of isolation, alienation, exclusion, or embarrassment resulting from being different in some way.

Stigma is a prominent factor that sets assistive technology apart from medical devices or mainstream products. Stigma associating with the assistive products is closely linked to appearance or the level of assistance associated with the product (Norman, 2002). Vaes, Stapper, Standaert, and Desager distinguish between four types of stigma:

1. Visible stigma, like wheelchair users, hearing aides etc.

2. Hidden stigma (such as HIV/aids).

3. Achieved stigma (homeless people, prisoners).

4. Voluntary stigma (piercings, political symbols, extreme fashion)(Vaes, Stapper, Standaert \& Desager, 2012).

When stroke patients use an external device to assist them to achieve a goal, it should be considered that stroke patients will receive a visible stigma when using adaptive products. Visible stigma can have an adverse effect on identity and will cause a conflict between users and many assistive products (Skogsrød, n.d.). 
The challenge of stigma research explores techniques to measure and quantify how "burdened" product and design concepts are with product-related stigma. Product-related stigma includes stigma-charged interactions and conflicts between products, users, bystanders and cultural factors (Vaes, 2014, p.19). Product-related stigma mainly occurs to people who are forced or have no choice to use products which are ignored by designers. The target group, like the elderly or people with disabilities mostly relies on assistive or medical devices that are more likely to experience stigma. For example, some AT devices are often overlooked by aesthetic views and popular design practice.

The stigma review of 'Product Stigmaticity' (Vaes, 2014) has provided a considerable structure that understands, measures and manages product-related stigma. Through analyzing a few examples, such as a wheelchair and dust mask, Vaes' research allows further exploration to investigate stigma elicited by products, especially in the recommendations of measuring product-related sigma (reactions to products-related stigma, empathy through sociability) and managing product-related stigma. The management of product-related stigma includes product-related stigma appraisal, outcomes of product-related stigma, and the evolution of product-related stigma over time (Vaes, 2014). 
Aesthetics, stigma and usability are closely connected when it relates to AT. The standard definition of a useless or ugly product relates that except for the functionality, the connection between aesthetics and stigma makes people feel "more disabled" (Skogsrød, n.d.). The World Health Organization recognizes disability "as a complex interaction between features of a person's body and features of the environment and society in which he or she lives" (World Health Organization, 2001). The book Design Meets Disability (Pullin, 2009) has been an inspiration for research contributions in the field of inclusive design. Pullin presents a thorough exploration of the cultural, stylistic and fashion trends that influence the acceptance of objects that overcome 'disability'. In one article, Susanne Jacobsen explores three categories for overcoming the stigma associated with assistive devices (Jacobson, 2010). The three categories disguise the stigmatizing features, turn attention from stigmatizing features to other features, and transform stigmatizing features into features that convey prestige or status.

In recent years, the emotional impact of products on the positive side of the acceptance spectrum has been widely studied. Norman (2004) states that the emotional side of design might be more critical to a product's success than its practical elements. In Jordan's study (1996), lack of usability, poor performance, lack of reliability and poor aesthetics were the main factors associated with unsatisfactory products. Associated feelings aroused were annoyance/irritation, anxiety/insecurity, contempt, and exasperation. The 'displeasure' feelings and emotional responses associated with the human-product interaction are my primary concern.

Marzano (1998) stated that products create a relationship with people when they work as living objects. Products can make people happy or angry, proud or ashamed, secure or anxious. In the consideration of product functionality and contextual environment, I will particularly concentrate on inclusive design, product semantics and human-centred design when generating the design process. 


\subsection{Design review}

Cutlery evolution in history

Sets of knifes, spoons, forks have been passed on from generation to generation all over the globe, travelling the whole world as pieces of personal history. The development of cutlery has paralleled human progress throughout history. Each generation of culinary implements represents a development of table manners and cutlery culture. To achieve the goal of understanding the significance of cutlery in humans' lives, a historical analysis of cutlery evolution is adopted by timeline and literature collections.
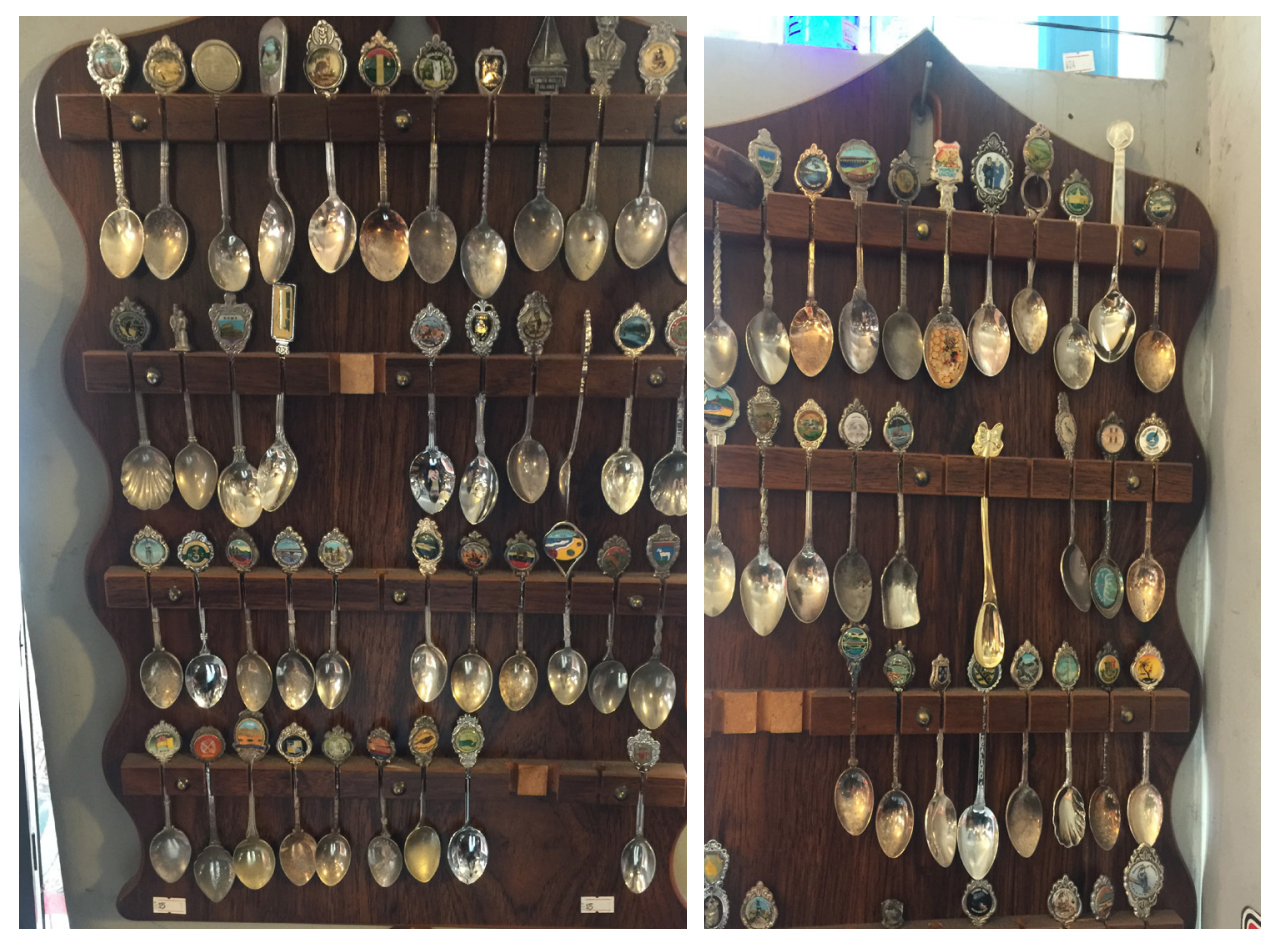

Figure 1.1 Affected hand (finger,thumb, wrist) (BOTOX, 2017) 


\section{BC}

\section{Technology}

Smelting malachite to extract copper.

Stone and copper used in tandem.

Metal was too soft and could not hold a sharp edge.

Later, the discovery of bronze, an alloy of copper with tin, brought a strong material that was easier to cast and could be hardened by hammering.

\section{Context}

Romans arrived with their Bronze- and Iron-age culture, and produced knives that tended to be short and straight, and were perhaps more practical than ornate.

Northumbrian monks forged a style of knife blade known as the scramasax, which became popular throughout Europe. One of the design characteristics of this knife was the broadness of the $b$ lade and the dramatic 'dropped point', whether for a small knife or a full-length blade of a sword.
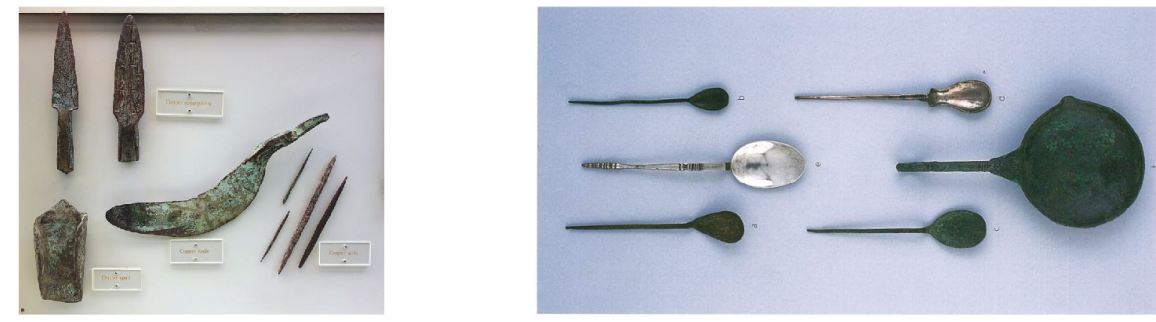

\section{$1000 A D$}

\section{Context}

In Europe, the Norman created fine table manners, rich tableware and implements. Spoons evolved from the Anglo-Saxon version of the Roman metal to the long French metal style.

By the end of fourteenth century, knives were more close to a practical shape and many knives were fitted with composite handles and decorated with patterns of alloy rivet. Spoons of the thirteenth century usually had a fig-like bowl.
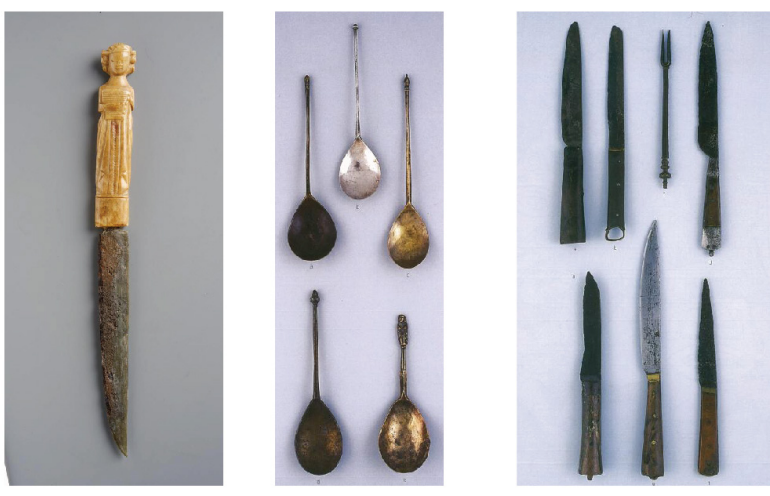

\section{0}

\section{Context}

Cutlery started being fashioned Europe and around the world. Knives were usually constructed with narrow, elegant blades and had decorative alloy caps and bolsters. The bolster changed from a copper alloy component soldered to an integral forged iron bolster. Spoons progressed from long handled with a small bowl to the 'first' spoon.
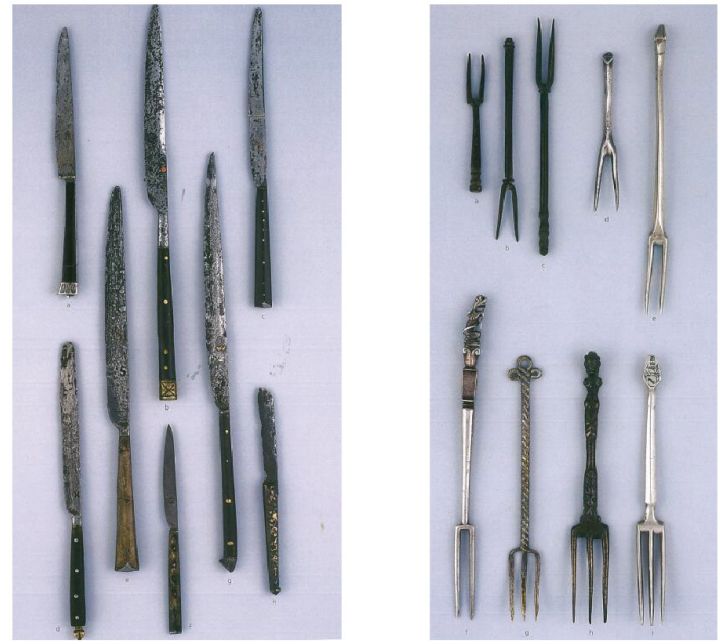


\section{0}

\section{Context}

The eating knives of the sixteenth century had been thin and elegant, whereas seventeenth century blades were shorter and with a rounded rip. Handles were becoming much more decorative, having composite examples in carved ivory, jet, ebony, amber, agate and precious metal. Cutlery has started producing pairs of knives in a decorated sheath.

By the end of century, sets of cutlery started being produce in higher numbers.

Significant changes occurred in the shapes of knife handles and blades. The blades became slightly curved with a simple bolster, eventually evolving into a scimitar-like shape with distinctive 'flying' bolster. The handle was fashioned in a range of
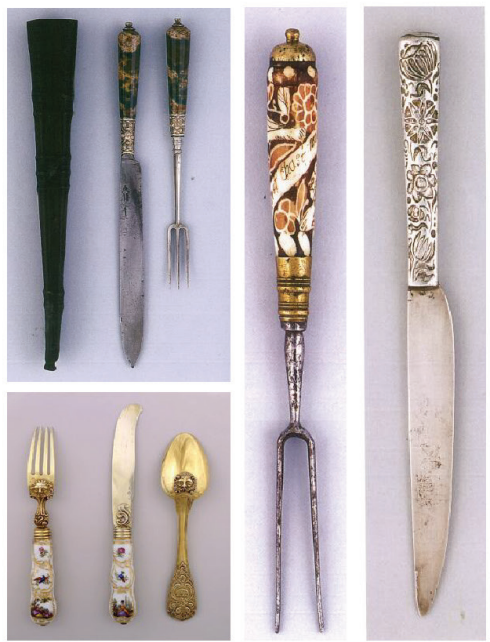
exotic materials and started to take on a pistol-grip shape.

\section{0}

\section{Material involved}

Massy silver, agate (France, Sheffield), ebony, ivory, green fish skin, amber tortoise shell, exotic glass.

\section{Design characters}

Ceramic handle imported into England. Straight-sided or pistol shapes.

\section{Context}

In the middle of this century, scimitar style changes came to French-style blades. Spear-point blades and straight-sided handle were replaced by simpler parallel-side blade.

By the end of this period, cutlery for special purposes made its appearance. Mechanical cheese scoops and masticating knives were two of the innovations.
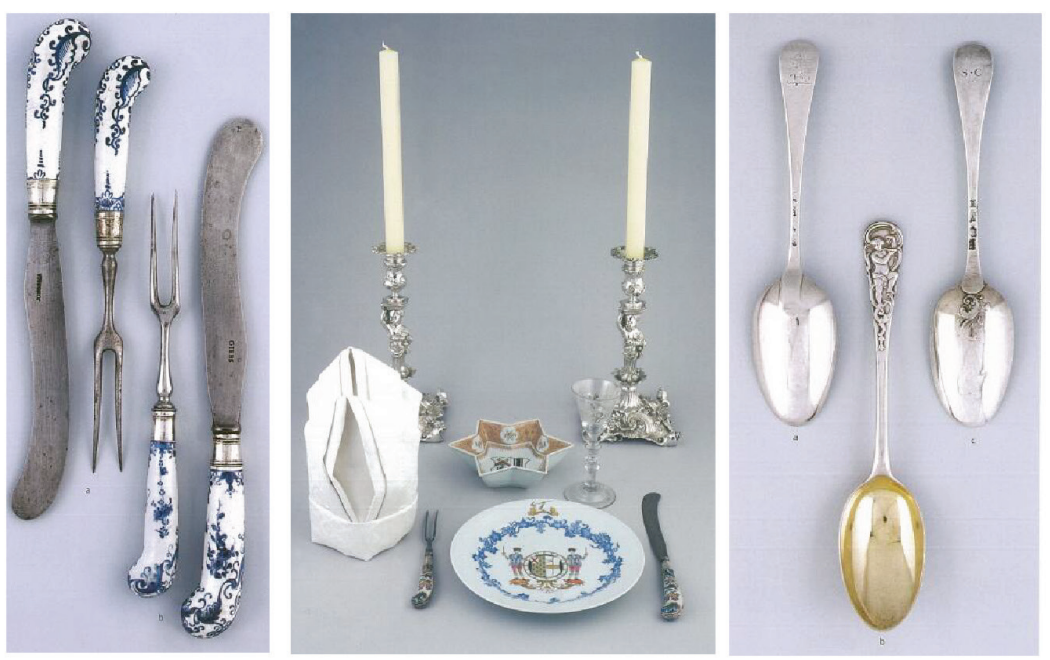

\section{0}

\section{Material involved \\ Celluloid}

\section{Design characters}

A large proportion of handles of antler or ivory.

\section{Context}

Beautiful sets of cutlery and personalized products. A number of companies made boxes for sets of dessert knives and forks.

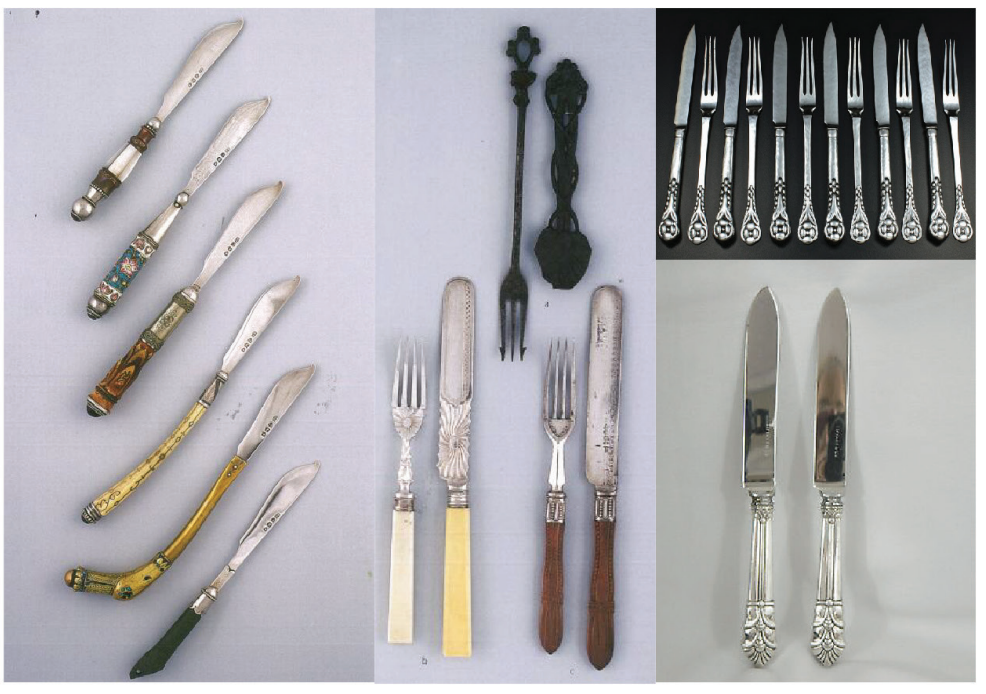




\section{0-1950}

\section{Primary Summary}

1. Celluloid handles, imitating natural products.

2. Commemorative spoons.

3. Brearley discovered stainless steel in Sheffield in 1914. In 1920, a small number of

cutlers started to produce knives with blades of stainless steel.

\section{$1950-2000$}

\section{Context}

1. Carl Pott Junior created an entirely new line of cutlery oriented towards the

Bauhaus.

2. Knife blades and spoon bowls became smaller. For spoons, the egg-shape turned round by 180 degrees, to ensure easier access to the mouth.

3. Pieces of cutlery used to be on average more than $24 \mathrm{~cm}$ long; the new size represented a reversion to $20-22 \mathrm{~cm}$ (This trend comes from Scandinavian novelties, after the Second World War).

4. Knife blades became shorter, but the handles were longer .

Examples: '81'\& '83'

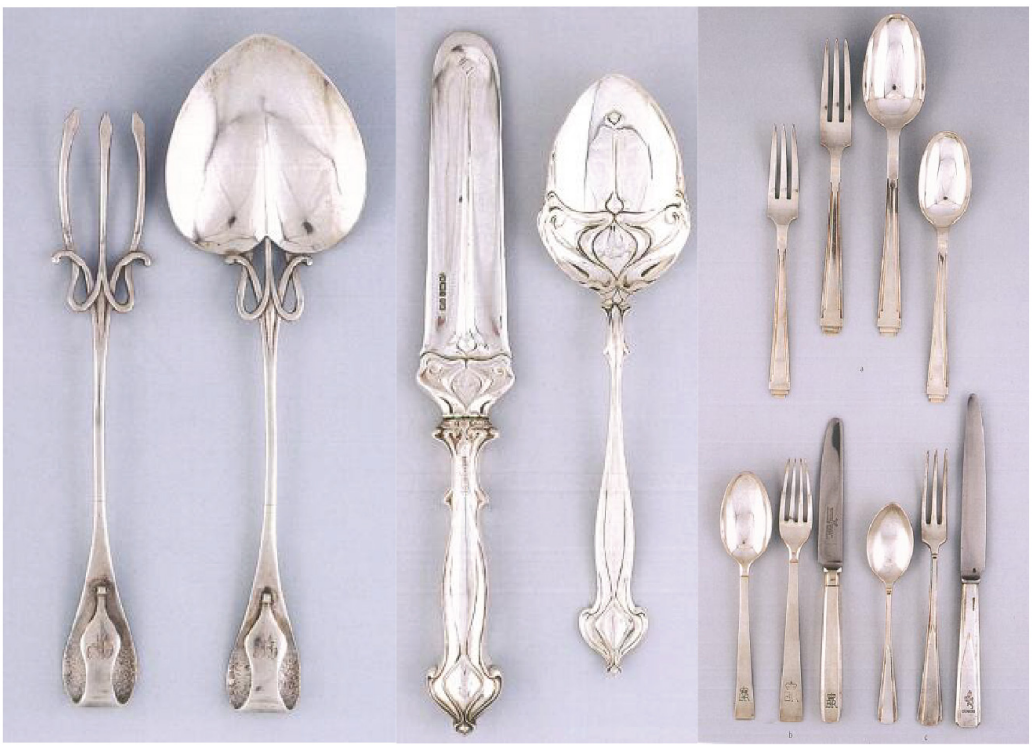

\section{8-1960}

\section{Context}

1. Soft line emerged in post-war Europe and predominated utensils about the 1960 s.

2. Both models had handles with rounded terminals.

3. Forms become softer; styles were fluid (provided purchase for fingers, making it comfortable and easy to hold the utensil).

4. The knife hafts and the handles of the other pieces had a slight groove that ran to the back. This groove or depression had a radius that was pressed inwards - by then a widespread method of designing handles.

5. Some traditional patterns still produced on cutlery products, but elaborate the manufacturing process was more expensive than smooth form ware.

Examples: Model 'Bavaria 658', Ernst Moeckl.

\section{0}

\section{Context}

1. Trapezoidal principles: Little lips above the rounded edge, supposed to prevent dripping.

2. These models were not designed with ergonomics but tended to feel unusual in the hand. 'Model composition S'.

3. The trapezoid-line shows that 20th century trends and fashions prevailed for shorter periods.

4. Household appliances with stringently square-tipped and angular forms (example: 'Mono-a').

5. Fundamental flat, broad, formalistic design.

6. 'New York', by Henning Kopped, made use of elementary geometric forms: circles, squares and cubes. He replaced curve with the straight line. 


\section{0}

\section{Context}

Background

The effects of social development since 1968.The first oil crisis and a worldwide recession.

1. Forms that had been deliberately designed as angular were now round in the extreme.

2. The 'mono-clip' handles had applied plastic panels. The oval spoon bowls were oriented towards the circular.

Examples: mono-clip/Chinese Ivory

The cross-section of the fork was fully integrated in the stripes, a typical soft line solution.

3. A new type of design emerged in mid-1970s cutlery, with handles consisting of a single broad strip of stainless steel. The width of the handle determined the breadth of the bowl and blades.

\section{0}

Context

Coloured plastic handles, unconventional handles of material, colour and form.

Designer: Lino Sabattini.

'Sculptura 18100' and 'Instrumenta'.

Background: Postmodern architecture and social trend (the gourmet craze).

Expression of a new style: luxury.-Example: 'Moisson' from Hermes,1985.

Heavy version

Maxing Rue Royale model by Plewe Cardin.

'Celeration'at home; pleasant tactile quality'.

'Onda' and 'Nuovo Milano'.

\section{0}

Context

New-Wave-since 1994

1. Handles are no longer straight and stiff but instead are often arc-shaped in contour.

2. 'Grand PrixAC08' Alessi

3. 'Chinese Ivory' David Mellor.

4. Zick

High tech and freestyle

Technical possibility: Sheet metal and forging.

Examples:

'Mono-clip'' Peter Raacke (sheet metal).

'Design 2 Don Wallance (Forging).

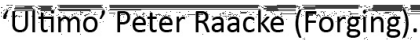

'7000'-by-Janos-Megyik.

The knife's strong virtual volume distinguished by a high aesthetic and ergonometric standard has been created by folding over the shape.

('Hana' Shozo Toyohisa) 
The history of cutlery also reflects an evolutionary history of human culture and technology. The cutlery history dates from the Stone Age, the Bronze Age, and the Iron Age until the stainless steel age. Most noteworthy is the early stage of the 20th century, as the widespread applications of electricity and the invention of stainless steel broke and reshaped traditional manufacturing patterns and the cutlery trade.

Today, the high resistance character of stainless steel has proved its advantage better than carbon steel. The cutlery steel manufacturers have fully integrated operations, computerized processes and control procedures that enable them to produce a higher quality medical and cutlery grade steel (Huebner,2002).

Beyond all doubt, advanced materials like titanium alloys, carbon fibres, new polyethylenes, plastic, synthetic fibres, and composite and advanced ceramics will reshape our lives in ways that we cannot imagine. 
Review of current market utensils

In the current market, there is a wide range of existing assistive technology utensil products that healthcare practitioners can choose from. However, most of the current cutlery products for patients do not consist of both usability and aesthetic features. In a study by Torrens (Torren \& Smith 2013), a user-centred design approach has been applied to the measurement of a range of existing AT cutlery products. For those patients with arthritis, Caring Cutlery is 'more appropriate' this condition (Torrens \& Smith, 2013, p.346). A conventional handle shape would be helpful for those with neuro-muscular conditions. In terms of effective functions, Etan Cutlery is more conventional looking, and also provides a useable high-friction grip surface and handles shape. Based on experiment, comments made and tacit observations during the assessments, elements that contribute to stigma that require addressing include an unusual shape coupled with a perceived association with medical products (Torren \& Smith, 2013). In addition, the conventional spoon bowl appears to require a deeper bowl for those with arthritis. However, a number of limitations in this assessment experiment have been identified, such as joint angles or direct force measurement. For a further proposal (Torrens \& Smith, 2013), the new design should address functional needs as well as the social function or value of the cutlery.

Torrens experiments of evaluation have been proved a significant contribution to develop AT cutlery sets. Few experiments and results of her studies are summarized as follows: 
Evaluation of an assistive technology product design

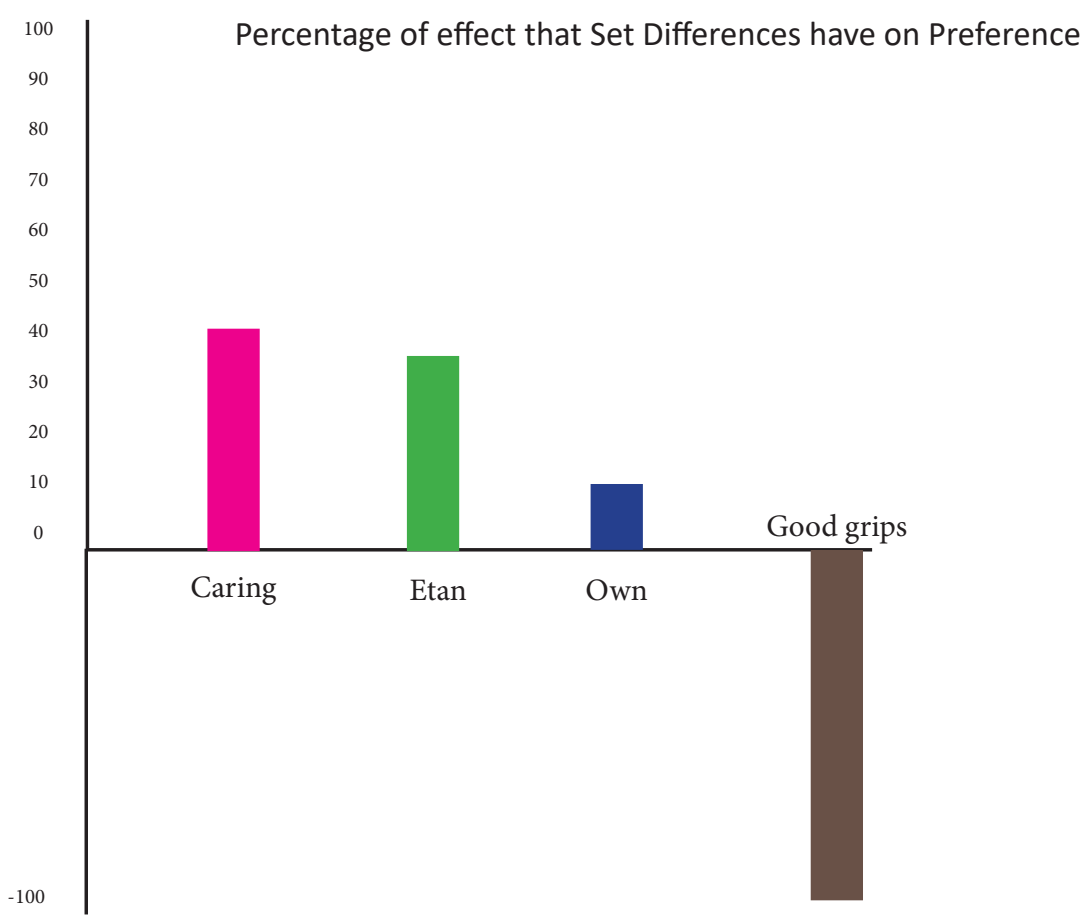

Figure 1.3 Adopted evaluation of four types AT cutlery with upper limb participant (Torrens \& Smith, 2013).
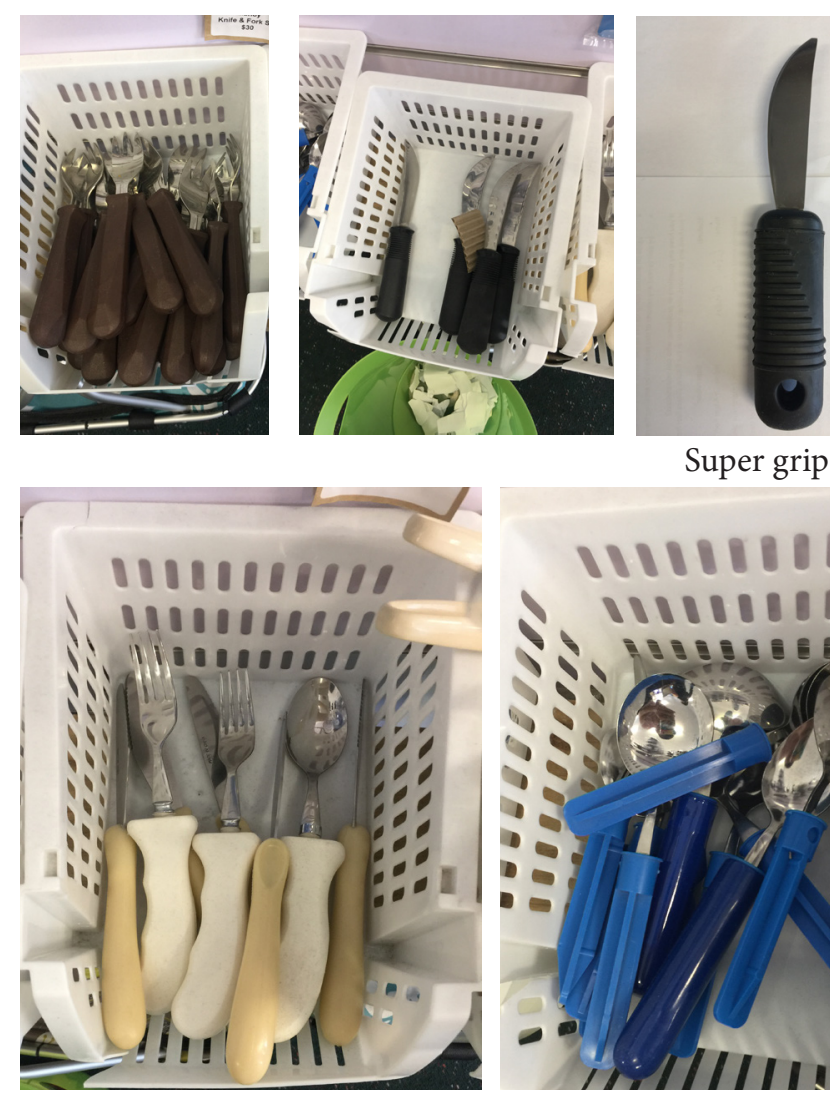

Super grip

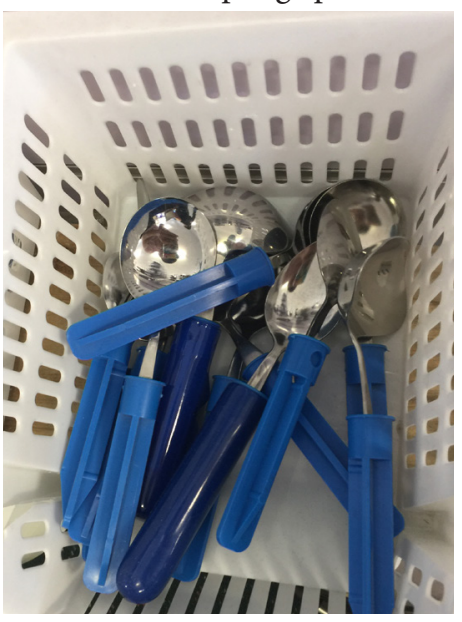

Figure 1.4 AT cutlery sets for disability 
'Getting a grip' experiment (Torrens, 2001) results analysis
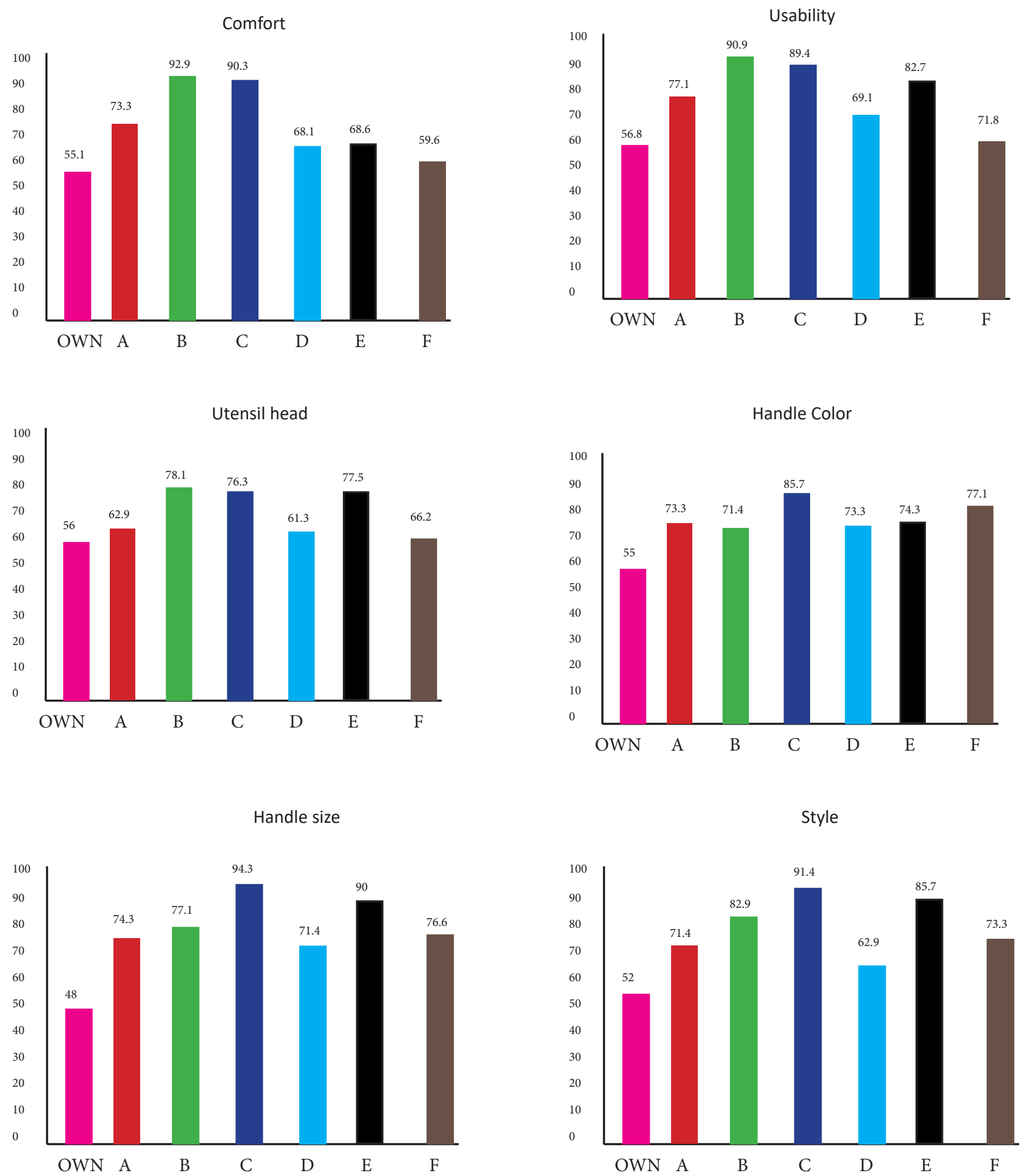

Figure 1.5 Adopted results of comparing 7 AT cutlery sets(Torrens \& McDonagh-Philp, 2001) 

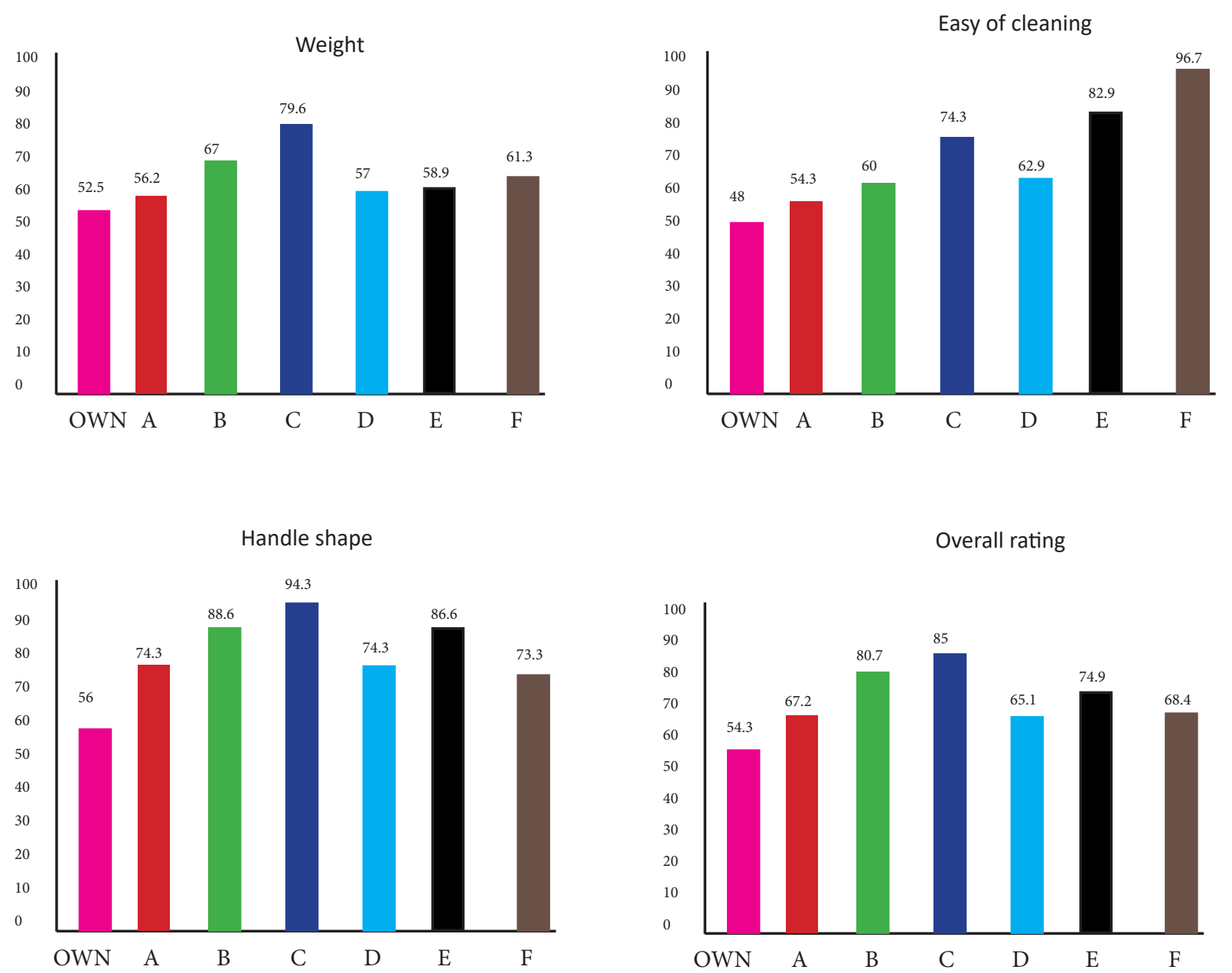

$\begin{array}{lll}\text { Sample } & \text { Name } & \text { Supplier } \\ \text { Own } & \text { Own cutlery } & \text { Smith\&Nephew Limited } \\ \text { A } & \text { Queens Cutlery } & \text { Nephew Nephew } \\ \text { B } & \text { AMEFA Cutlery } & \text { Nottingham Rehab Limited } \\ \text { C } & \text { Select-grip Cutlery } & \text { Nottingham Rehab Limited } \\ \text { D } & \text { Caring Cutlery } & \text { Nottingham Rehab Limited } \\ \text { E } & \text { Ultralite Cutlery } & \text { Nottingham Rehab Limited } \\ \text { F } & \text { Good Grip Cutlery } & \text { Nottingham Rehab Limited }\end{array}$




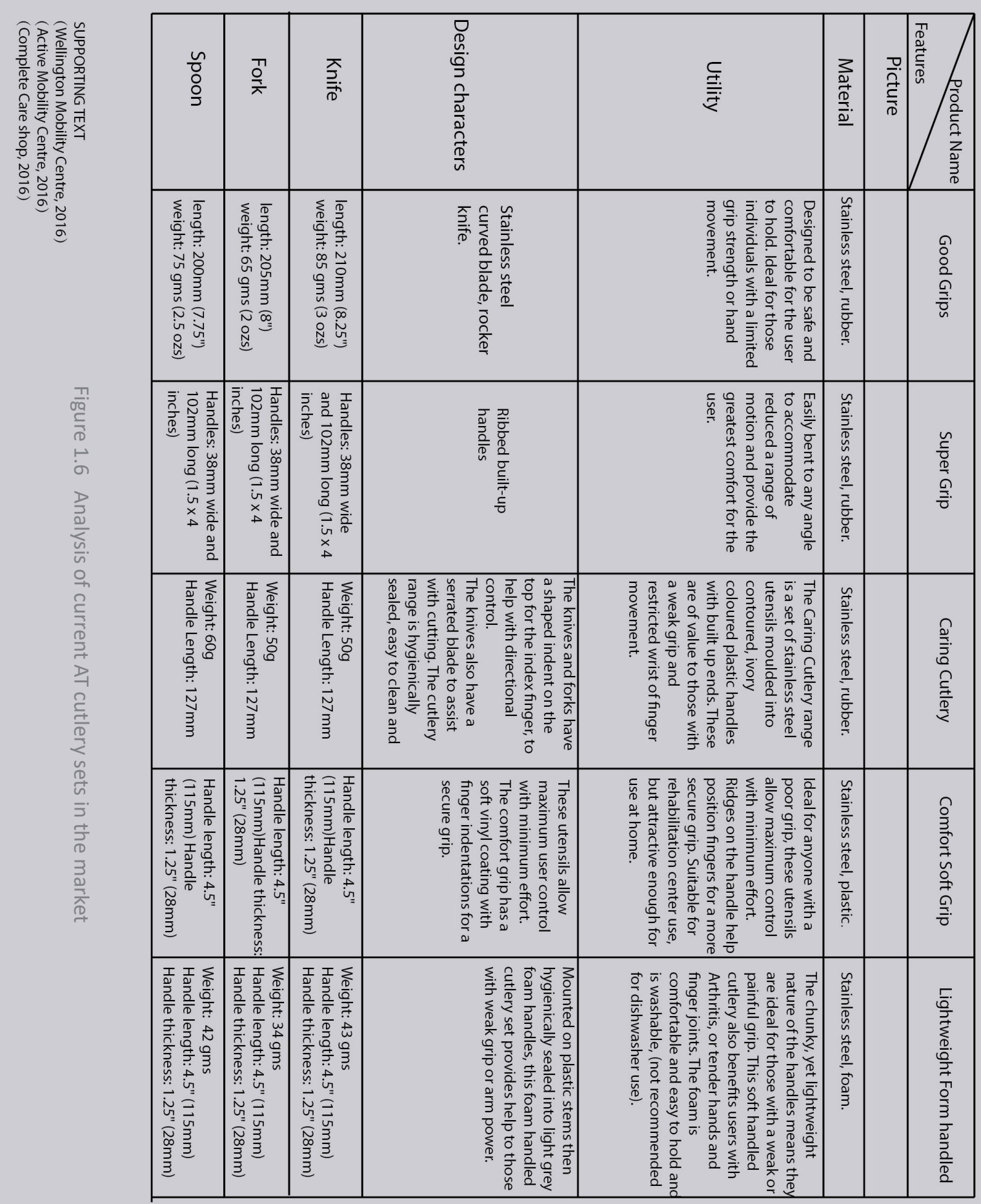



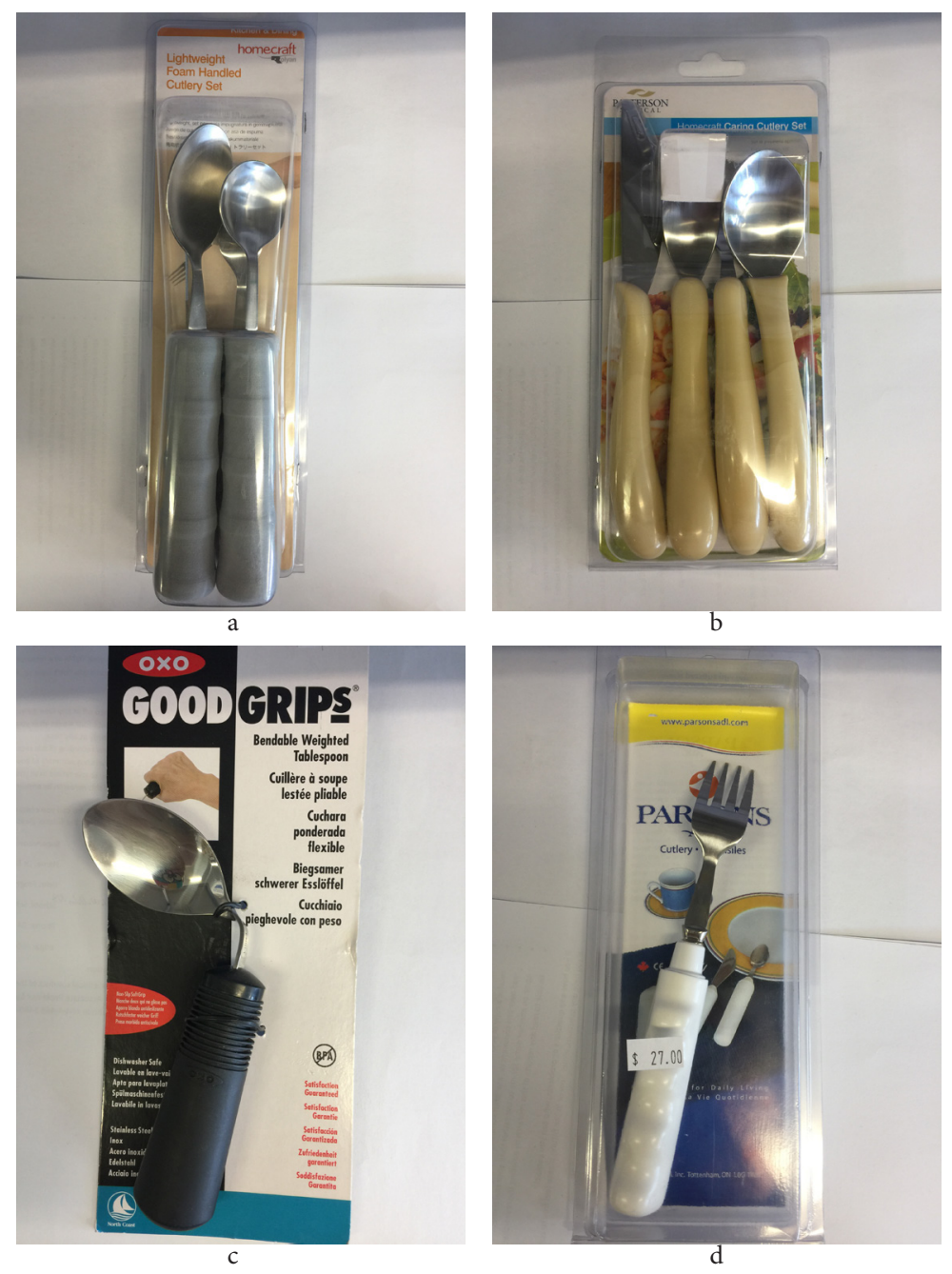

Figure 1.7 Light Weight cutlery (a), Caring Cutlery (b), Good Grip (c), Comfort Cutlery (d) 
Personal perspective

Lightweight

The character of foam is that it can match with patients' palm shape and provide various pressed shapes that also can transform back to the original. Foam material is easily affected by water wash, especially when the handled is stained by food. Although the official introduction of the foam cutlery set described will benefits users with inflamed hands and fingers joints, the feature of spasticity of stroke patients has not been specifically solved.

The Caring Cutlery

The fake skin colour would make users and bystanders experience uncomfortable emotions when they come into contact with the ivory coloured rubber. Comparing the material with other existing products, Caring Cutlery is the only set that separate handle shapes among fork, knife and spoon. But adding curved rubber handle is not enough when facing a stroke patient's uniquely affected hand.

\section{Super Grip}

Super Grip cutlery also called bendable cutlery by its bendable metal. The bendable material decides the cutlery shape can transform with consumer need, but it also reveals the problem behind this character. That is the designer was not bring the design intervention into this product, and leave more potential problems that seems like solved already. The bended angle of cutlery necks are easy to break by number of times and all handles are designed by a same shape.

\section{Good grip}

Similar with the other mobility cutlery products, the good grip also adopts bendable metal in the neck part. Adding additional material is for increasing contact area between palm and handle. All of these design concern are from the features that patient having gaps between their fingers and palms. In this design, the additional material of handle has been making the cutlery overweight, and easily to drop when patient use it in eating activity. 
Evaluation of existing adaptive cutlery by using Vaes' PAMS intervention

Following the literature review, this chapter aims to clearly show that instances of product-related stigma occur in real-life encounters (Vaes, 2014, p.134). These encounters engender both positive and negative emotions and reactions in users, bystanders and broader cultural contexts. The researcher will explore the factors responsible for eliciting these positive and negative experiences in people's encounters with current AT cutlery products. As such, this chapter contributes to two of the research goals that deepen understanding of product-related stigma (PRS) and prepare information to the designer to manage the PRS process.

The Product Appraisal Model for Stigma (PAMS) exposes and explains the context and appraisal factors that influence the product-related stigma process. Mainly, the PAMS try to alleviate the complexity of surrounding products, people and cultures. The PAMS was aimed at exploring the contextual and appraisal issues that surround the use of assistive, protective and medical devices. The outputs of PAMS are a list of stigma-specific design challenges in need of vital attention at the start of a stigma-specific design challenge.

In the next design research chapter, concerning the Product Intervention Model for Stigma (PIMS), the research will document the selected design interventions that can be applied to the stigma-specific challenges, as revealed by the PAMS. These interventions serve as a support to a designer managing stigma-sensitive design challenges, and they aim at reducing the PRS content of a new design proposal (Vaes, 2014, p.134).

Considering the contribution of PAMS in addressing stigma, an analysis of current AT utensils will be conducted by evaluating some representative products. The selected utensils for assessing and addressing stigma are Good Grip Cutlery, Caring Cutlery, and Comfort Grip Cutlery. 


\section{Product Perception (Sensing)}

Perceiving, playing with or caressing the product.

\section{Human attention}

\section{Attitudes Appraisal}

The stakeholder's prevailing tendency to like or dislike qualities of objects. people pr activities.

\section{Goals Appraisal}

"Things" that the stakeholder wants to accomplish or see happed. Goals provides orientation and purpose in human-product interaction
Does the product exhibit sensory discomforting or repelling features for its user? (Visual, noise, smell, tactile, taste)

A user experiences a claustrophobic feel when wearing a dust mask.
Does product perception obstruct the user in obtaining his or her goals?

When a user perceives a pair of crutches for the first time, does the product 'look' reveal to its user how to achieve the goal of walking with crutches?
Does the product exhibit a sensory discomforting or repelling impact on bystanders?

The unsuspected tactile impact when an unsuspected bystander shakes hands with a user of an artificial hand.

\section{Cultural}

Does product perception violate social or cultural taste?

Cultural attitudes regarding mini-skirts or piercings can be in conflict with or violate cultural taste.
Does product perception obstruct others in obtaining their goals?

Perceiving a user-product combination can induce avoidance/greater social distance in bystanders, conflicting with their goal of passing the user in a conventional way.
Does product perception obstruct a culture in obtaining its goals?

Certain political symbols/ signs can obstruct or undermine cultural and societal goals.

\section{Standards Appraisal}

The stakeholder's expectations and beliefs about how users, others and objects should behave or act. Standards refer to value.
Does the user perceive unacceptable products or product aspects according to his quality standards (physical, ergonomical, psychological, moral)?

A product can be devalued through negative brand associations or a negative institutional identity.
Do bystanders perceive products or product aspects of users that fail according to their quality standards: physically, ergonomically, psychologically, morally?

When bystanders perceive a wheelchair user, the encounter could elicit feelings of aversion, compassion, or admiration in the bystander.
Does the perceived cultural significance and value of the product or product aspects indicate change over time or in durability?

Public views have changed on the way people with braces are perceived. 


\section{Consequences of Product Use (evaluation and meaning)}

\begin{tabular}{|c|c|c|c|}
\hline $\begin{array}{l}\text { Fantasizing about, } \\
\text { remembering, or } \\
\text { anticipating usage } \\
\text { or consequences of } \\
\text { usage }\end{array}$ & User & Bystander & Cultural \\
\hline $\begin{array}{l}\text { Attitudes Appraisal } \\
\text { The stakeholder's } \\
\text { prevailing tendency } \\
\text { to like or dislike } \\
\text { qualities of objects. } \\
\text { people pr activities. }\end{array}$ & $\begin{array}{l}\text { Does the look and feel of } \\
\text { the product match with the } \\
\text { personality and lifestyle of } \\
\text { its user? } \\
\text { Product semantics and } \\
\text { product meaning need to } \\
\text { complement the attitudes } \\
\text { of the product user. }\end{array}$ & $\begin{array}{l}\text { Does the look and feel of the } \\
\text { product and its user match } \\
\text { with the attitudes of the } \\
\text { bystander? } \\
\text { A product that matches } \\
\text { its user's personality can } \\
\text { refer to a subculture or } \\
\text { group that is disapproved of } \\
\text { or rejected, e.g. extreme } \\
\text { piercings. }\end{array}$ & $\begin{array}{l}\text { Does the look and feel } \\
\text { of the product and its } \\
\text { user match with cultural } \\
\text { preferences? } \\
\text { What are the stereotypical } \\
\text { cultural habits associated } \\
\text { with the product that shape } \\
\text { this cultural evaluation? }\end{array}$ \\
\hline $\begin{array}{l}\text { Goals Appraisal } \\
\text { "Things" that the } \\
\text { stakeholder wants } \\
\text { to accomplish or see } \\
\text { happed. Goals } \\
\text { provides orientation } \\
\text { and purpose in } \\
\text { human-product } \\
\text { interaction }\end{array}$ & $\begin{array}{l}\text { Is the product tolerated } \\
\text { purely out of necessity or } \\
\text { physical dependency? }\end{array}$ & $\begin{array}{l}\text { Do the consequences of } \\
\text { product use harm the } \\
\text { physical or psychological } \\
\text { integrity of others? }\end{array}$ & $\begin{array}{l}\text { Cultural and societal goals } \\
\text { should be met. Are the } \\
\text { product and its features } \\
\text { in compliance with these } \\
\text { goals and the supporting } \\
\text { regulations? } \\
\text { Individual aspirations } \\
\text { are secondary to societal } \\
\text { goals. Fake products are } \\
\text { 'outlaws'. A fake Rolex watch } \\
\text { can be linked to a lower } \\
\text { social class, thus conflicting } \\
\text { with the goal of its user to } \\
\text { elicit status. }\end{array}$ \\
\hline $\begin{array}{l}\text { Standards Appraisal } \\
\text { The stakeholder's } \\
\text { expectations and } \\
\text { beliefs about how } \\
\text { users, others and } \\
\text { objects should } \\
\text { behave or act. Stand- } \\
\text { ards refer to value. }\end{array}$ & $\begin{array}{l}\text { Does the product } \\
\text { conflict with the user's } \\
\text { expectations and beliefs } \\
\text { following product use? } \\
\text { People often carry out } \\
\text { 'after sales checks' of their } \\
\text { recently bought products. } \\
\text { E.g. a new car. }\end{array}$ & $\begin{array}{l}\text { Does the product conflict } \\
\text { with the bystander's expec- } \\
\text { tations and beliefs following } \\
\text { product use? } \\
\text { During or after their } \\
\text { encounter with a product } \\
\text { user, bystanders can be } \\
\text { confronted with their own } \\
\text { vulnerability. }\end{array}$ & $\begin{array}{l}\text { Does the product conflict } \\
\text { with the cultural values, } \\
\text { expectations and beliefs } \\
\text { following product use? } \\
\text { How can the avoidance of } \\
\text { conflict be turned into the } \\
\text { opposite - a hype? How can } \\
\text { it be turned into a product } \\
\text { that is the result of a high } \\
\text { generative level of creation } \\
\text { and supported by the full } \\
\text { blessing of culture and } \\
\text { society? }\end{array}$ \\
\hline
\end{tabular}




\section{Consequences of Product Use (evaluation and meaning)}

\begin{tabular}{|c|c|c|c|}
\hline $\begin{array}{l}\text { Fantasizing about, } \\
\text { remembering, or } \\
\text { anticipating usage } \\
\text { or consequences of } \\
\text { usage }\end{array}$ & User & Bystander & Cultural \\
\hline $\begin{array}{l}\text { Attitudes Appraisal } \\
\text { The stakeholder's } \\
\text { prevailing tendency } \\
\text { to like or dislike } \\
\text { qualities of objects. } \\
\text { people pr activities. }\end{array}$ & $\begin{array}{l}\text { Does the look and feel of } \\
\text { the product match with the } \\
\text { personality and lifestyle of } \\
\text { its user? } \\
\text { Product semantics and } \\
\text { product meaning need to } \\
\text { complement the attitudes } \\
\text { of the product user. }\end{array}$ & $\begin{array}{l}\text { Does the look and feel of the } \\
\text { product and its user match } \\
\text { with the attitudes of the } \\
\text { bystander? } \\
\text { A product that matches } \\
\text { its user's personality can } \\
\text { refer to a subculture or } \\
\text { group that is disapproved of } \\
\text { or rejected, e.g. extreme } \\
\text { piercings. }\end{array}$ & $\begin{array}{l}\text { Does the look and feel } \\
\text { of the product and its } \\
\text { user match with cultural } \\
\text { preferences? } \\
\text { What are the stereotypical } \\
\text { cultural habits associated } \\
\text { with the product that shape } \\
\text { this cultural evaluation? }\end{array}$ \\
\hline $\begin{array}{l}\text { Goals Appraisal } \\
\text { "Things" that the } \\
\text { stakeholder wants } \\
\text { to accomplish or see } \\
\text { happed. Goals } \\
\text { provides orientation } \\
\text { and purpose in } \\
\text { human-product } \\
\text { interaction }\end{array}$ & $\begin{array}{l}\text { Is the product tolerated } \\
\text { purely out of necessity or } \\
\text { physical dependency? }\end{array}$ & $\begin{array}{l}\text { Do the consequences of } \\
\text { product use harm the } \\
\text { physical or psychological } \\
\text { integrity of others? }\end{array}$ & $\begin{array}{l}\text { Cultural and societal goals } \\
\text { should be met. Are the } \\
\text { product and its features } \\
\text { in compliance with these } \\
\text { goals and the supporting } \\
\text { regulations? } \\
\text { Individual aspirations } \\
\text { are secondary to societal } \\
\text { goals. Fake products are } \\
\text { 'outlaws'. A fake Rolex watch } \\
\text { can be linked to a lower } \\
\text { social class, thus conflicting } \\
\text { with the goal of its user to } \\
\text { elicit status. }\end{array}$ \\
\hline $\begin{array}{l}\text { Standards Appraisal } \\
\text { The stakeholder's } \\
\text { expectations and } \\
\text { beliefs about how } \\
\text { users, others and } \\
\text { objects should } \\
\text { behave or act. Stand- } \\
\text { ards refer to value. }\end{array}$ & $\begin{array}{l}\text { Does the product } \\
\text { conflict with the user's } \\
\text { expectations and beliefs } \\
\text { following product use? } \\
\text { People often carry out } \\
\text { 'after sales checks' of their } \\
\text { recently bought products. } \\
\text { E.g. a new car. }\end{array}$ & $\begin{array}{l}\text { Does the product conflict } \\
\text { with the bystander's expec- } \\
\text { tations and beliefs following } \\
\text { product use? } \\
\text { During or after their } \\
\text { encounter with a product } \\
\text { user, bystanders can be } \\
\text { confronted with their own } \\
\text { vulnerability. }\end{array}$ & $\begin{array}{l}\text { Does the product conflict } \\
\text { with the cultural values, } \\
\text { expectations and beliefs } \\
\text { following product use? } \\
\text { How can the avoidance of } \\
\text { conflict be turned into the } \\
\text { opposite - a hype? How can } \\
\text { it be turned into a product } \\
\text { that is the result of a high } \\
\text { generative level of creation } \\
\text { and supported by the full } \\
\text { blessing of culture and } \\
\text { society? }\end{array}$ \\
\hline
\end{tabular}




\section{Product Perception (Sensing)(Good Grip)}

\begin{tabular}{|c|c|c|c|}
\hline $\begin{array}{l}\text { Perceiving, playing } \\
\text { with or caressing the } \\
\text { product. } \\
\text { Human attention }\end{array}$ & User & Bystander & Culture \\
\hline $\begin{array}{l}\text { Attitudes Appraisal } \\
\text { The stakeholder's } \\
\text { prevailing tendency to } \\
\text { like or dislike qualities of } \\
\text { objects. people pr } \\
\text { activities. }\end{array}$ & $\begin{array}{l}\text { Does the product exhibit } \\
\text { sensory discomforting or } \\
\text { repelling features for its } \\
\text { user? (Visual, noise, smell, } \\
\text { tactile, taste) } \\
\text { The rubber material discom- } \\
\text { forts user's hand and the } \\
\text { front bendable side is an } \\
\text { unsightly features for user. }\end{array}$ & $\begin{array}{l}\text { The bendable elements } \\
\text { discomfort bystander by } \\
\text { visual sensory. }\end{array}$ & $\begin{array}{l}\text { A bended spoon and fork } \\
\text { might violate a social taste } \\
\text { in a table manner. }\end{array}$ \\
\hline $\begin{array}{l}\text { Goals Appraisal } \\
\text { "Things" that the stake- } \\
\text { holder wants to accom- } \\
\text { plish or see happed. } \\
\text { Goals provides orienta- } \\
\text { tion and purpose in } \\
\text { human-product interac- } \\
\text { tion }\end{array}$ & $\begin{array}{l}\text { Does product perception } \\
\text { obstruct the user in } \\
\text { obtaining his or her goals? } \\
\text { It does not, it provides a } \\
\text { bendable angle for a patient } \\
\text { who have wrist and shoulder } \\
\text { difficulty. Anti-slip elements } \\
\text { also increase the finger } \\
\text { friction with handle. The } \\
\text { bendable also require a } \\
\text { strength folding actions that } \\
\text { can not finish by a elderly } \\
\text { patient. }\end{array}$ & $\begin{array}{l}\text { Does product perception } \\
\text { obstruct others in } \\
\text { obtaining their goals? } \\
\text { The rubber material might be } \\
\text { sticky for clean, which } \\
\text { obstruct in obtaining wash } \\
\text { goals. }\end{array}$ & $\begin{array}{l}\text { Does product perception } \\
\text { obstruct a culture in } \\
\text { obtaining its goals? }\end{array}$ \\
\hline $\begin{array}{l}\text { Standards Appraisal } \\
\text { The stakeholder's } \\
\text { expectations and beliefs } \\
\text { about how users, others } \\
\text { and objects should } \\
\text { behave or act. Stand- } \\
\text { ards refer to value. }\end{array}$ & $\begin{array}{l}\text { Does the user perceive } \\
\text { unacceptable products or } \\
\text { product aspects according } \\
\text { to his quality standards } \\
\text { (physical, ergonomical, } \\
\text { psychological, moral)? } \\
\text { Except the physical stand- } \\
\text { ards, all the others aspects, } \\
\text { as well as aesthetic, ergo- } \\
\text { nomic, psychological of this } \\
\text { product are not meet the } \\
\text { designer expectations. }\end{array}$ & $\begin{array}{l}\text { Do bystanders perceive prod- } \\
\text { ucts or product aspects of } \\
\text { users that fail according to their } \\
\text { quality standards: physically, } \\
\text { ergonomically, psychologically, } \\
\text { morally? } \\
\text { Good grips cutlery will affect } \\
\text { the bystander expectation of } \\
\text { an adaptive cutlery. They will } \\
\text { understand the bendable } \\
\text { function, but might be have } \\
\text { different advices to the } \\
\text { design. } \\
\text { When bystanders perceive } \\
\text { stroke patient, some might } \\
\text { feel compassion. But in } \\
\text { contrast, a lot of people } \\
\text { reported that the elicit } \\
\text { feelings of admiration for } \\
\text { being open and amicable, } \\
\text { regardless of patient's' condi- } \\
\text { tions. }\end{array}$ & $\begin{array}{l}\text { Does the perceived cultural } \\
\text { significance and value of } \\
\text { the product or product } \\
\text { aspects indicate change } \\
\text { over time or in durability? }\end{array}$ \\
\hline
\end{tabular}




\section{Product in Use (Acting)(Good Grip)}

\section{Human intentions with products: using, operating or manag- ing products}

\section{Attitudes Appraisal}

The stakeholder's prevailing tendency to like or dislike qualities of objects. people pr activities.

\section{Goals Appraisal}

"Things" that the stakeholder wants to accomplish or see happed. Goals provides orientation and purpose in human-product interaction

\section{Standards Appraisal}

The stakeholder's expectations and beliefs about how users, others and objects should behave or act. Standards refer to value.

\section{Is the product}

discomforting or repelling during its use (in general or in specific situations)? Applying and removing

For patient have wrist pain and shoulder lift difficulty, a bendable elements is easier to take food independently. A large handle can fit the palm where grip weakness

Does the product fit its purpose of use, physically, functionally, ergonomically, and morally?

It might fit the purpose of use in physically., but not in others sides

Are there any dissonant experiences (unbalances between thinking/feeling and acting) when using the product?

It might have dissonant experiences about the color and sticky material.
Does using the product interfere negatively with the behavior of others; does it prevent others from attaining their goals?

The patient domestic members or hospital association will ask to be help them bend the cutlery neck side, which demand might be interfere their own eating process.

Does using the product challenge the tolerance of bystanders?

For some people don't agree this bendable design, it does challenge the tolerance of bystanders.

\section{Culture}

Does using the product conflict with cultural habits, rules or laws?

It will conflict a table manner and visual effect of eating.

Does using the product indicate inappropriate cultural or societal behavior?

It may not.

Does the introduction of the product still need to overcome thresholds in view of cultural or social acceptability?

When more people use adaptive cutlery in public, this ritual becomes widespread, plausibly influencing its cultural acceptance. 


\section{Consequences of Product Use (evaluation and meaning)(Good Grip)}

\begin{tabular}{|c|c|c|c|}
\hline $\begin{array}{l}\text { Fantasizing about, } \\
\text { remembering, or } \\
\text { anticipating usage or } \\
\text { consequences of usage }\end{array}$ & User & Bystander & Culture \\
\hline $\begin{array}{l}\text { Attitudes Appraisal } \\
\text { The stakeholder's } \\
\text { prevailing tendency } \\
\text { to like or dislike } \\
\text { qualities of objects. } \\
\text { people pr activities. }\end{array}$ & $\begin{array}{l}\text { Does the look and feel of } \\
\text { the product match with the } \\
\text { personality and lifestyle of its } \\
\text { user? } \\
\text { It matches with their } \\
\text { disabilities for patients, and } \\
\text { it should not express the } \\
\text { personality in that way. }\end{array}$ & $\begin{array}{l}\text { Does the look and feel of } \\
\text { the product and its user } \\
\text { match with the attitudes of } \\
\text { the bystander? }\end{array}$ & $\begin{array}{l}\text { Does the look and feel } \\
\text { of the product and its } \\
\text { user match with cultural } \\
\text { preferences? } \\
\text { when prompted to "help } \\
\text { himself", patient will need to } \\
\text { carry the cutlery by them- } \\
\text { selves. In certain cultures, this } \\
\text { could be considered as } \\
\text { disrespectful. }\end{array}$ \\
\hline $\begin{array}{l}\text { Goals Appraisal } \\
\text { "Things" that the stake- } \\
\text { holder wants to accom- } \\
\text { plish or see happed. } \\
\text { Goals provides orienta- } \\
\text { tion and purpose in } \\
\text { human-product interac- } \\
\text { tion }\end{array}$ & $\begin{array}{l}\text { Is the product tolerated } \\
\text { purely out of necessity or } \\
\text { physical dependency? } \\
\text { The patient can tolerate the } \\
\text { product with a unique } \\
\text { appearance, because their } \\
\text { pathological conditions } \\
\text { require the cutlery to be } \\
\text { designed with large } \\
\text { handles and anti-slip } \\
\text { materials. }\end{array}$ & $\begin{array}{l}\text { Do the consequences of } \\
\text { product use harm the } \\
\text { physical or psychological } \\
\text { integrity of others? } \\
\text { Bystander will have concern } \\
\text { and compassion after they } \\
\text { know the pathological } \\
\text { condition of patient. }\end{array}$ & $\begin{array}{l}\text { Cultural and societal goals } \\
\text { should be met. Are the } \\
\text { product and its features } \\
\text { in compliance with these } \\
\text { goals and the supporting } \\
\text { regulations? } \\
\text { Cutlery functions have } \\
\text { met the requirements. }\end{array}$ \\
\hline $\begin{array}{l}\text { Standards Appraisal } \\
\text { The stakeholder's } \\
\text { expectations and } \\
\text { beliefs about how users, } \\
\text { others and objects } \\
\text { should behave or act. } \\
\text { Standards refer to value. }\end{array}$ & $\begin{array}{l}\text { The product have been } \\
\text { provided a basic function } \\
\text { for satisfied user expecta- } \\
\text { tion, but it is not present a } \\
\text { good design for customer } \\
\text { use. }\end{array}$ & $\begin{array}{l}\text { Does the product conflict } \\
\text { with the bystander's } \\
\text { expectations and beliefs } \\
\text { following product use? }\end{array}$ & $\begin{array}{l}\text { Does the product conflict } \\
\text { with the cultural values, } \\
\text { expectations and beliefs } \\
\text { following product use? }\end{array}$ \\
\hline
\end{tabular}




\section{Product Perception (Sensing)(Comfort Cutlery)}

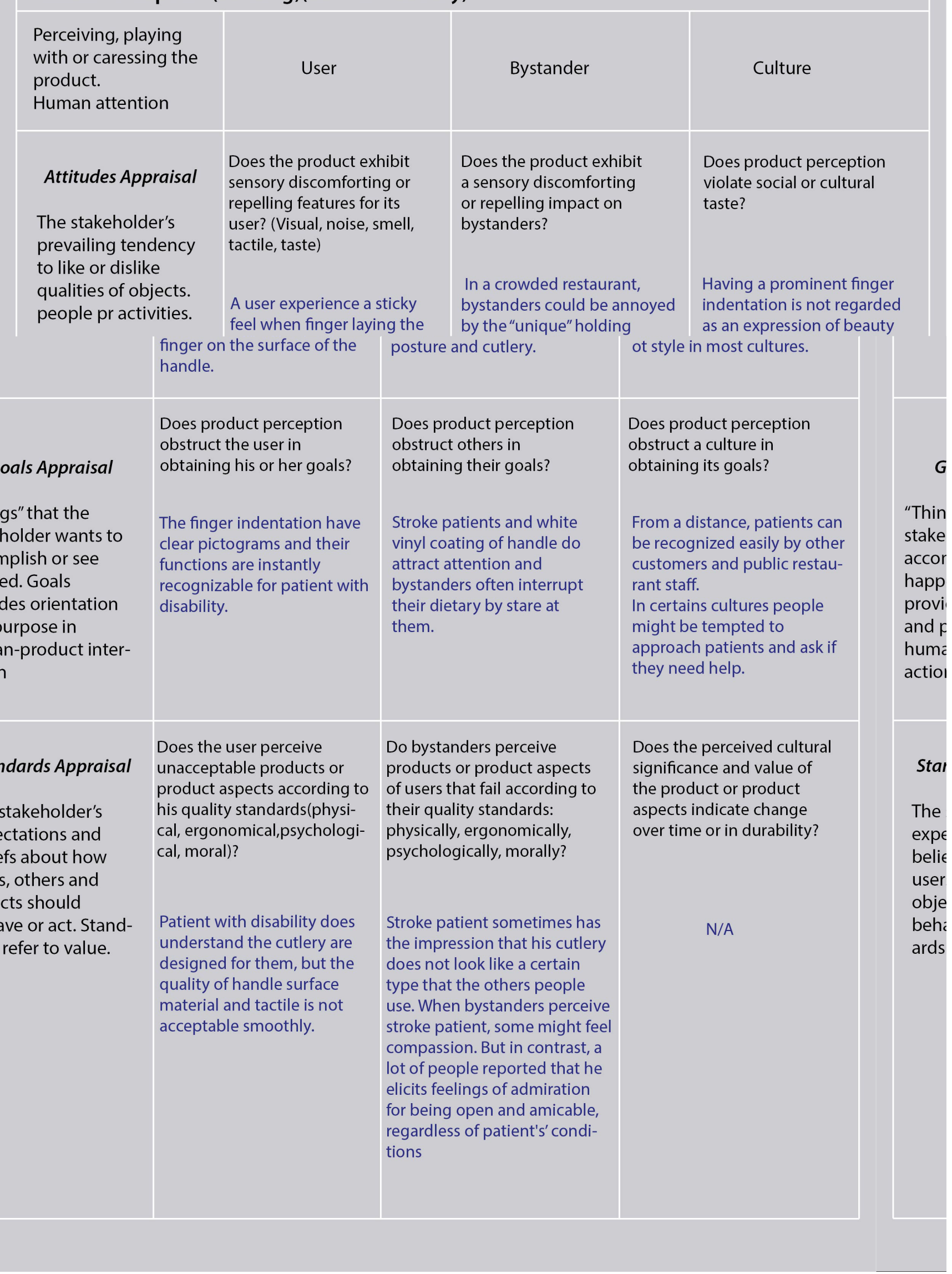




\section{Product in Use (Acting)(Comfort Cutlery)}

Human intentions with products: using, operating or managing products

\section{Attitudes Appraisal}

The stakeholder's prevailing tendency to like or dislike qualities of objects. people pr activities.

\section{Goals Appraisal}

"Things" that the stakeholder wants to accomplish or see happed. Goals provides orientation and purpose in human-product interaction

\section{Standards Appraisal}

The stakeholder's expectations and beliefs about how users, others and objects should behave or act. Standards refer to value.
Is the product discomforting or repelling during its use (in general or in specific situations)? Applying and removing

Fit the patient palm with uncomfortable hold. The stereotype, as so called "ergonomic" does not provide the function like it introduced.The white vinyl are easily to be dirty and absorbing small baffeta.

Does the product fit its purpose of use, physically, functionally, ergonomically, and morally?

The cutlery can provide a basic function such as cutting, scooping and pricking food from plate. However, in a dietary taking, use also want keep their table as good as possible. But the finger indentations are not interact with four fingers well during it use.

Are there any dissonant experiences (unbalances between thinking/feeling and acting) when using the product?

Except the from knife edge, spoon bowl, and fork prong are look similar with the other cutlery sets, all of the handle are regarded as to be use in no mood.
Does using the product cause unease or a threat to others?

Fitting a paralysed upper limb may cause unease in bystanders. Patient with disabilities often senses that bystanders maintain a greater distance.

\section{Culture}

Does using the product conflict with cultural habits, rules or laws?

Patient will not be ask to use the normal cutlery sets, because their family and clinicians will encourage them to use assistive device.
Does using the product interfere negatively with the behavior of others; does it prevent others from attaining their goals?

Bystander are easily to be aware of patient upper limb difficulties, and after they touched their cutlery sets, bystander did focus watching their hands and dont want eat with them together.

Does using the product challenge the tolerance of bystanders?

Due to the patient's age and level of difficulty, bystander would perhaps make their right thought.
Does using the product indicate inappropriate cultural or societal behavior?

For the table manner, the patient should be used to see their hands getting dirty by dropped food. For instance, the patient unstable hand dropping the knife or fork will interrupt the frequency of eating.

Does the introduction of the product still need to overcome thresholds in view of cultural or social acceptability?

When more people use adaptive cutlery in public, this ritual becomes widespread, plausibly influencing its cultural acceptance. 


\section{Consequences of Product Use (evaluation and meaning)(Comfort Cutlery)}

\begin{tabular}{|c|c|c|c|}
\hline $\begin{array}{l}\text { Fantasizing about, } \\
\text { remembering, or } \\
\text { anticipating usage or } \\
\text { consequences of usage }\end{array}$ & User & Bystander & Culture \\
\hline $\begin{array}{l}\text { Attitudes Appraisal } \\
\text { The stakeholder's } \\
\text { prevailing tendency } \\
\text { to like or dislike } \\
\text { qualities of objects. } \\
\text { people pr activities. }\end{array}$ & $\begin{array}{l}\text { Does the look and feel of } \\
\text { the product match with the } \\
\text { personality and lifestyle of } \\
\text { its user? }\end{array}$ & $\begin{array}{l}\text { Does the look and feel of } \\
\text { the product and its user } \\
\text { match with the attitudes of } \\
\text { the bystander? }\end{array}$ & $\begin{array}{l}\text { When prompted to "help } \\
\text { himself", patient will need } \\
\text { to carry the cutlery by } \\
\text { themselves. In certain } \\
\text { cultures this could be } \\
\text { considered as disrespectful. }\end{array}$ \\
\hline $\begin{array}{l}\text { Goals Appraisal } \\
\text { "Things" that the } \\
\text { stakeholder wants to } \\
\text { accomplish or see } \\
\text { happed. Goals } \\
\text { provides orientation } \\
\text { and purpose in } \\
\text { human-product } \\
\text { interaction }\end{array}$ & $\begin{array}{l}\text { The patient can tolerates } \\
\text { the product with unique } \\
\text { appearance, because their } \\
\text { pathological conditions } \\
\text { require the handle to be } \\
\text { designed with large } \\
\text { handle and anti-slip mate- } \\
\text { rials. }\end{array}$ & $\begin{array}{l}\text { Do the consequences of } \\
\text { product use harm the } \\
\text { physical or psychological } \\
\text { integrity of others? }\end{array}$ & $\begin{array}{l}\text { Cultural and societal goals } \\
\text { should be met. Are the } \\
\text { product and its features } \\
\text { in compliance with these } \\
\text { goals and the supporting } \\
\text { regulations? }\end{array}$ \\
\hline $\begin{array}{l}\text { Standards Appraisal } \\
\text { The stakeholder's } \\
\text { expectations and } \\
\text { beliefs about how } \\
\text { users, others and } \\
\text { objects should behave } \\
\text { or act. Standards refer } \\
\text { to value. }\end{array}$ & $\begin{array}{l}\text { The product have been } \\
\text { provided a basic func- } \\
\text { tion for satisfied user } \\
\text { expectation, but it is not } \\
\text { present a good design } \\
\text { for use. }\end{array}$ & $\begin{array}{l}\text { Does the product conflict } \\
\text { with the bystander's } \\
\text { expectations and beliefs } \\
\text { following product use? }\end{array}$ & $\begin{array}{l}\text { The cutlery set can use } \\
\text { independently either at } \\
\text { home or hospital. But it also } \\
\text { depends on the level of } \\
\text { difficulty they have. }\end{array}$ \\
\hline
\end{tabular}




\section{Product Perception (Sensing) (Caring Cutlery)}

Perceiving, playing with or caressing the product.

Human attention

\section{Attitudes Appraisal}

The stakeholder's prevailing tendency to like or dislike qualities of objects. people pr activities.

\section{Goals Appraisal}

"Things" that the stakeholder wants to accomplish or see happed. Goals provides orientation and purpose in human-product interaction

\section{Standards Appraisal}

The stakeholder's expectations and beliefs about how users, others and objects should behave or act. Standards refer to value.
Does the product exhibit sensory discomforting or repelling features for its user? (Visual, noise, smell, tactile, taste)

The caring cutlery exhibits sensory discomforting use by its handle color where can be recognized as fake skin color.

Does product perception obstruct the user in obtaining his or her goals?

The curve of the handle can make the user obtaining their goals.

Does the user perceive unacceptable products or product aspects according to his quality standards(physical, ergonomical,psychological, moral)?

The front side of cutlery presents a good quality of metal to user, it designed as well as an elegant cutlery image.
Does the product exhibit a sensory discomforting or repelling impact on bystanders?

A fake skin color would attract the bystander attention and cause their responses.

\section{Does product perception} obstruct others in obtaining their goals?

Patients who hold the caring cutlery do attract attention, and bystanders often avoid sitting next to them in the restaurant.

\section{Do bystanders perceive} products or product aspects of users that fail according to their quality standards: physically, ergonomically, psychologically, morally?

The caring cutlery does not look like others cutlery have an obvious color or assistive elements for help patients eat. It designed with ergonomic principles and access curve to fit affected palm cap. When bystanders perceive patient, some might feel compassion, in contrast, a lot of people reported that it elicits a feeling of admiration for being so open and positive, regardless of his/her condition.

\section{Culture}

Does product perception violate social or cultural taste?

Having additional materials in the handle and especially an unsightly color are not regarded as an expression of cutlery culture.

Does product perception obstruct a culture in obtaining its goals?

It does not.

Does the perceived cultural significance and value of the product or product aspects indicate change over time or in durability?

The streamline style of cutlery started from 1950s, when the globalization and pop culture are the main trend. 


\section{Product in Use (Acting)(Caring Cutlery)}

Human intentions with
products: using, oper-
ating or managing
products

Attitudes Appraisal

The stakeholder's prevailing tendency to like or dislike qualities of objects. people pr activities.

\section{Goals Appraisal}

"Things" that the stakeholder wants to accomplish or see happed. Goals provides orientation and purpose in human-product interaction
Is the product

discomforting or repelling during its use (in general or in specific situations)?

Applying and removing

It depends on user need and the level of grip difficulty.But in general, it provides a good curve to hold.

Does the product fit its purpose of use, physically, functionally, ergonomically, and morally?

It does serve the purpose of use in physically. But in function aspect, it can not meet the requirement that all category of patients who have disability.

\section{Standards Appraisal}

The stakeholder's expectations and beliefs about how users, others and objects should behave or act. Standards refer to value.
Are there any dissonant experiences (unbalances between thinking/feeling and acting) when using the product?

Uncertain the dissonant experience when using.
Does using the product interfere negatively with the behavior of others; does it prevent others from attaining their goals?

No, it does not affect the bystanders attaining their goals.

\section{Culture}

Does using the product conflict with cultural habits, rules or laws?

The fáke skin color might conflict with some the others cutlery in a table manner.

Does using the product indicate inappropriate cultural or societal behavior?

It might against some domestic table manners, but not against a particular culture.
Does using the product challenge the tolerance of bystanders?

No, it does not.
Does the introduction of the product still need to overcome thresholds in view of cultural or social acceptability?

When more people use the adaptive cutlery in public, this ritual becomes widespread, plausibly influencing its cultural acceptance. 


\section{Consequences of Product Use (evaluation and meaning)(Caring Cutlery)}

Fantasizing about, remembering, or anticipating usage or consequences of usage

User
$\begin{aligned} & \text { Does the look and feel of } \\ & \text { the product match with the } \\ & \text { personality and lifestyle of } \\ & \text { its user? }\end{aligned}$

The stakeholder's prevailing tendency to like or dislike qualities of objects. people pr activities.

\section{Goals Appraisal}

"Things" that the stakeholder wants to accomplish or see happed. Goals provides orientation and purpose in human-product interaction

\section{Standards Appraisal}

The stakeholder's expectations and beliefs about how users, others and objects should behave or act. Standards refer to value.
The caring cutlery set is neatly styled compared to others adaptive cutlery sets. It has a color option that be select by a customer preference.

Is the product tolerated purely out of necessity or physical dependency?

For a patient have upper limb difficulty, the user should tolerate the physical dependency.
Does the look and feel of the product and its user match with the attitudes of the bystander?

Although the cutlery are not present an aesthetically appearance, it did make the bystander know the basic image of products.

Do the consequences of product use harm the physical or psychological integrity of others?

Bystander will have concerns and compassion after they know the pathological condition of patient.

Does the product conflict with the bystander's expectations and beliefs following product use?

It will conflict with bystanders' expectation, but will not conflict with their beliefs
Does the look and feel of the product and its user match with cultural preferences?

It might not match with a elegant table manner or any domestic manner.

Cultural and societal goals should be met. Are the product and its features in compliance with these goals and the supporting regulations?

The additional material feature has been recognized not supporting a cutlery design regulations, and there is a large capacity of aesthetic need to be fill.

Does the product conflict with the cultural values, expectations and beliefs following product use?

The affected upper limb is often associated with domestic members and a hospital environment. There associations are shared by many and could be tough to alter. 


\subsection{Design precedents}

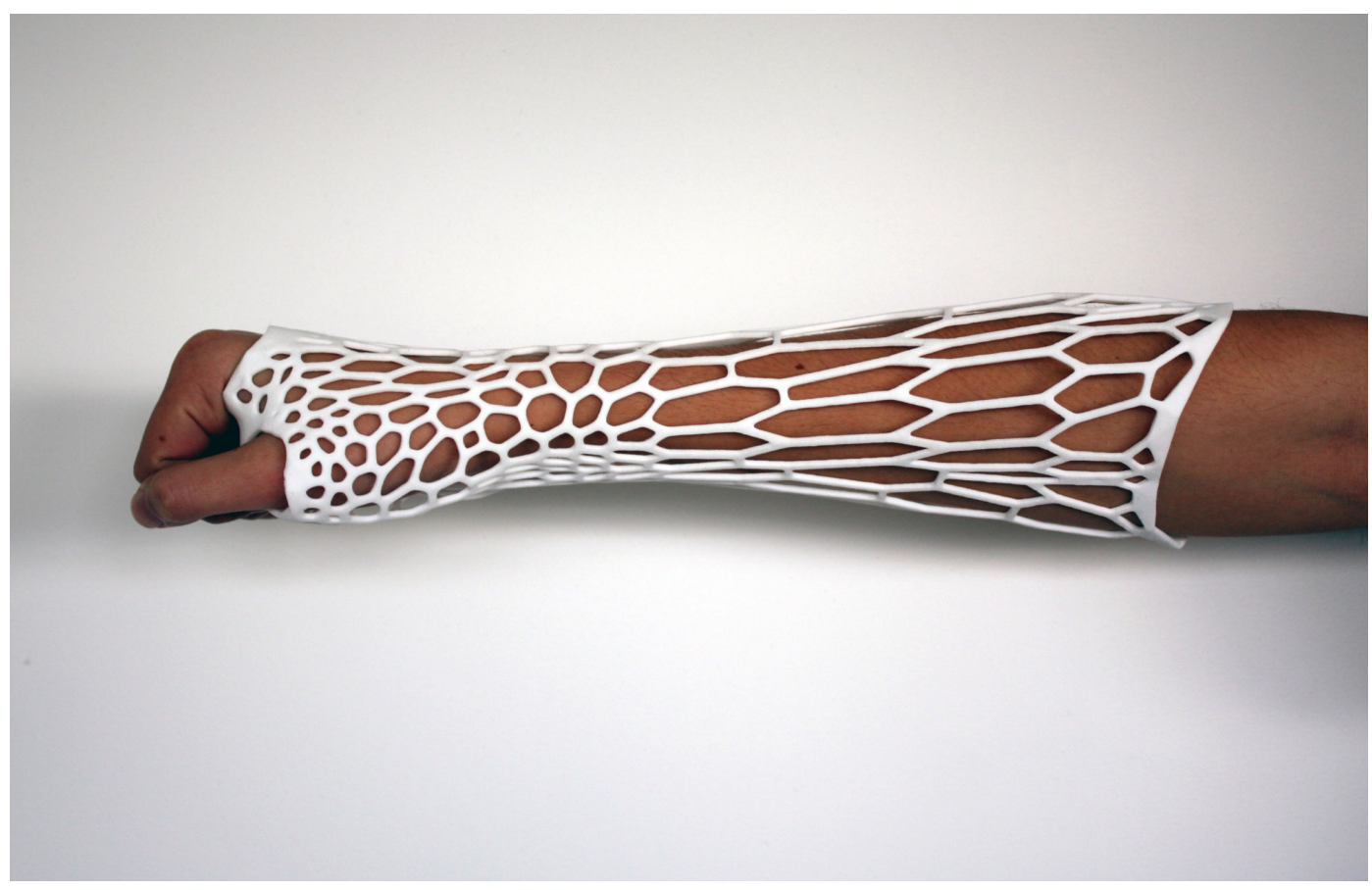

The Cortex exoskeletal cast provides a new design perspective in challenging the traditional cast. Not only light and stylish, the cortex cast utilizes the 3D-scan into the design process, which enables the user to customize a cast about the point of fracture (Evill, 2013). By a rapid increasing and advanced technology, 3D printing provides a significant opportunity to those products with diversified demands.

3D printing technology is at an inflection point with the designer rapidly adopting 3D print modelling to inform prototypes. 3D printing is tightly integrated with 3D modelling software; each file that is sent to a 3D printer can be modified or customized by using a variety of software-based customization tools (Micallef, 2015).

Figure 1.20 3D print cast: Cortex (Evill, 2013). 


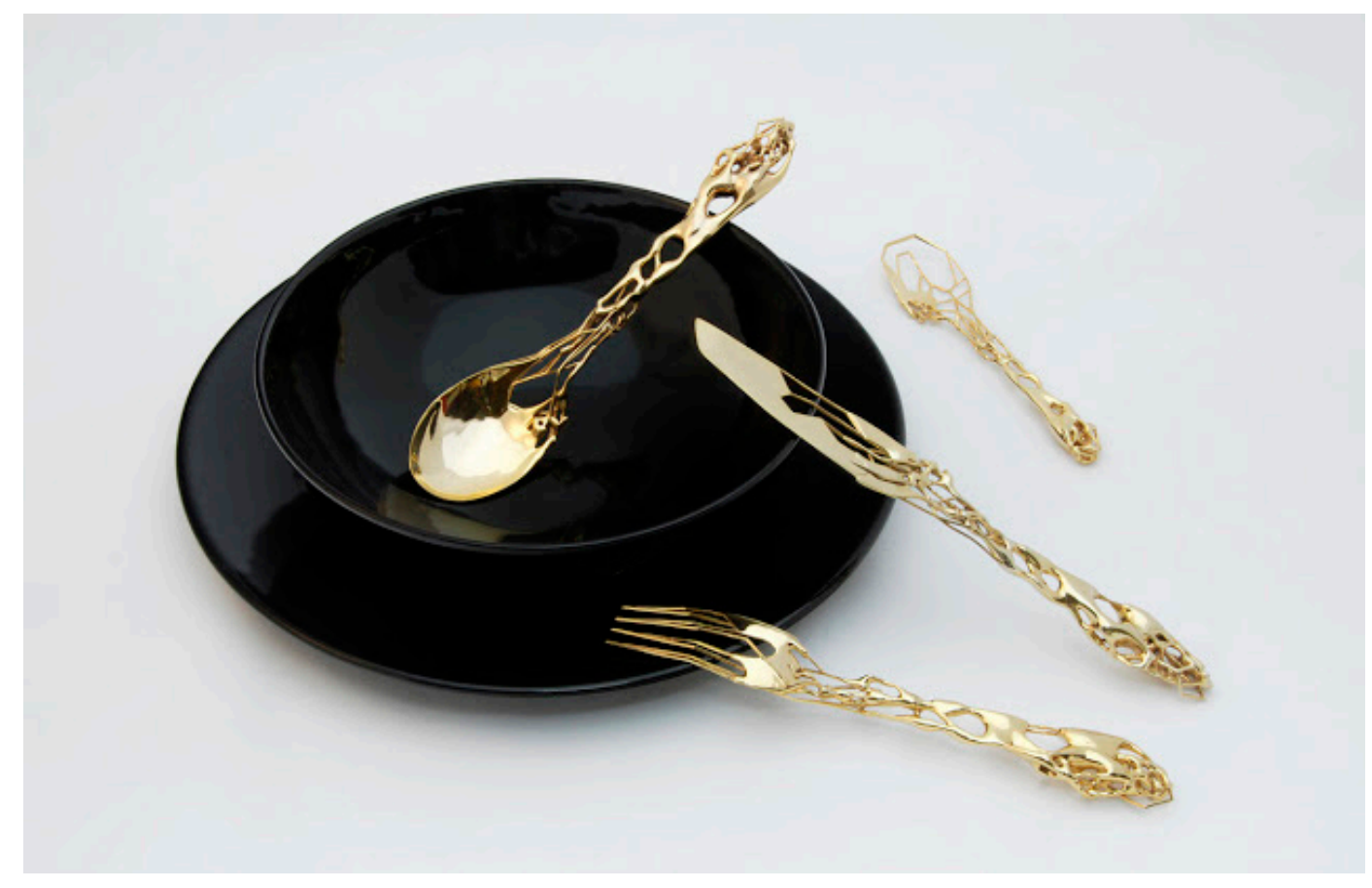

With the development of material and 3D printing technology, the scope of 3D printing design is increasing. It can be said that increasingly improved 3D print and user requirements bring a new challenge to traditional product design. For example, a metallic printer could produce a cutlery set that uses gold material (figure 1.21). The cutlery set would be produced by $18 \mathrm{kt}$ gold plated brass. 3D printing gives customers the chance to create their own designs. The Shapeways Company has applied a commercial pattern that allows users to upload their digital files and get their printing after payment. 

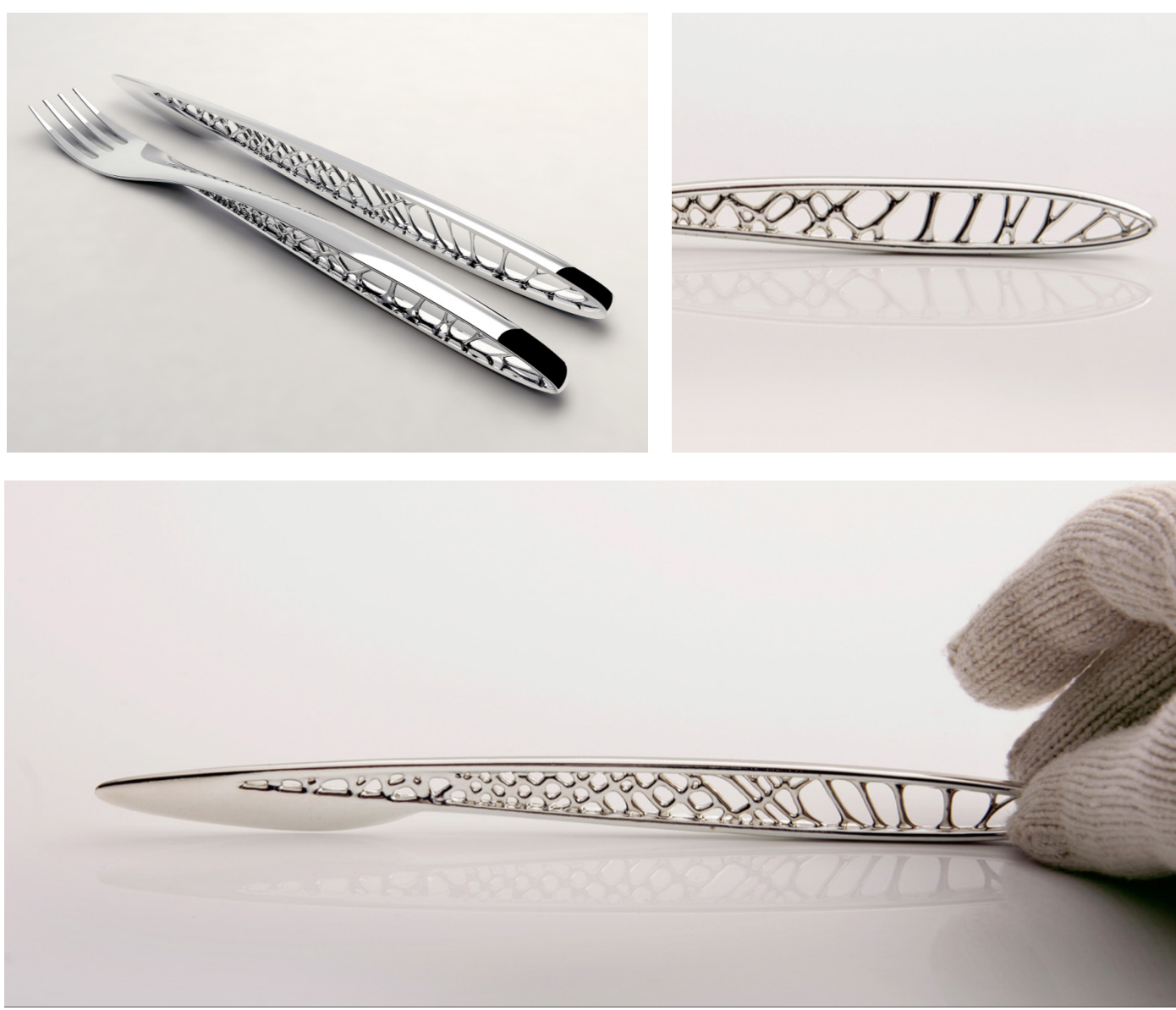

Aside from the functional aspect of tableware, cutlery has been ornamental, and it "brings a unique experience where all senses are stimulated in a seductive play of pure enjoyment" (LEAD, 2017). LEAD produces polished 3D-printed stainless cutlery (figure 1.22) by introducing a dynamic and organic formal language. "A modern geometric tension is brought into each object by disintegrating the stems with fluid lines that appear as a delicate, sensuous and elegant frozen liquid" (LEAD, 2017).

Figure 1.22 3D stainless steel print cutlery (LEAD, 2017). 
The product service system (Tran \& Park, 2015) has demonstrated that the customize design methodology in a specific product design will benefit customers, providers, the environment and the society. When designing a product for diversified customer demands, the customized methodology will enhance competitiveness and achieve the goal of sales volume. In the process of manufacturing a geometric shape and some prototypes with complexity, 3D printing plays an important role, mainly because it can facilitate a customized and speedy product with high accuracy, thus meeting the requirement of designing for customized or parametric shapes in a short time (Challies, 2015). Therefore, 3D printing enables custom manufacturing of personalized design into digital files, and it enables small quantities of customized products to be produced at relatively low cost.

There are two important areas of utensils to be aware of when designing: the handle portion and each utensil front portion. In view of more than one unit in the cutlery set, the cutlery body portion will be designed separately, such as the tines of the fork, the edges of the knife and the bowl of the spoon. With the goal of avoiding stigma, the following design consideration will focus on the functionality and contextual usability when designing the cutlery. Functionality includes the use of the cutlery to assist the eating motion. Contextual usability encompasses the ergonomics and aesthetic of the overall cutlery set. 



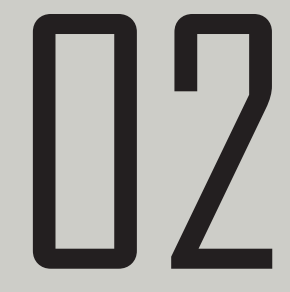

METHODS 
The purpose of this thesis is to develop a set of cutlery that can be used by people who are recovering from a stroke. This research addressed the functions the cutlery needs to emulate ergonomic and usability concerns of designing for stroke patients, as well as addressing the stigma of using medical devices in a contextual environment. The contextual environment can be divided into the domestic environment and public restaurants. This research was informed by a human-centred design methodology (Torrens, McDonagh-Philp, \& Newman, 2001), and provide foundations on which a prototype cutlery set is designed to help stroke patients eating at home or in public through interactions with an aesthetically considered set of cutlery.

In contrast to the situation that assistive products are easily abandoned, a process of collecting clinicians' insights about current assistive technology (AT) cutlery sets will be conducted. The clinicians consider the patients' ideologies and beliefs, as well as their physical and cultural environment (Duncan, 2016, p. 25)

\subsection{Research overview}

\section{Research questions}

How can a cutlery set assist stroke patients' eating with aesthetically-considered appearance?

\section{Hypothesis}

An aesthetically-considered cutlery set can help stroke patients with independent eating. 


\section{Aims and Objectives}

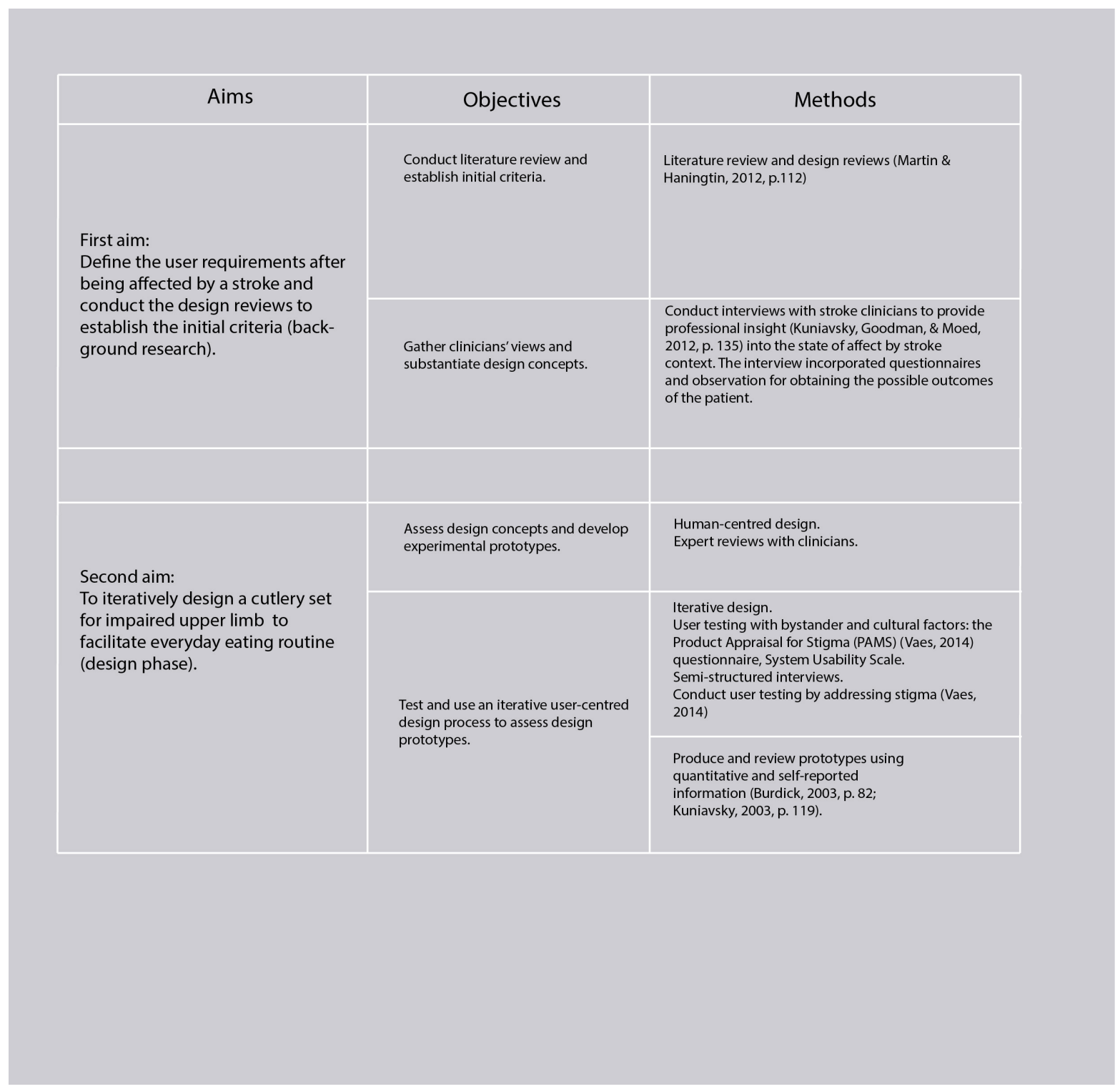

Figure 2.1 Aims and Objectives 


\subsection{User-centred design (UCD) research}

A user-centred design approach will reflect the patients' involvement and has been suggested by several papers as an indispensable reflection when developing a product for the patient. Stehle and Buehler (2008, p.109) propose that a user-centred design approach for developing more desirable products includes five stages:

1. Understand the problem and identify the opportunity.

2. Develop the concepts (this stage also focuses on translating the research findings from the first stage into product concepts).

3. Validate the concept (testing and determine whether product ideas are valid and feasible).

4. Refine the concept (improve design based on the third stage).

5. Commercialise the product (undergo engineering refinement and distribute the products). This is outside the scope of this thesis.

The background research informed the design phases through a combination of literature and design reviews (Martin \& Hanington, 2012, p.112). In the first stage, research began with using a combination of workshops, such as historical analysis of cutlery evolution and assessment of assistive cutlery. Also, much critical reflection supporting the criteria of the prototypes will come from clinicians' opinions and participant feedback. The second stage utilised sketching, iterative ideation and prototyping, as well as computer-aided design (CAD) modelling. This formed the body of the design phase and moving technique to develop the final outputs. In the final stage, the prototype was ready for clinical testing and feedback of the participants. To achieve the goals of understanding the user problem and identifying the opportunity of design, an online video analysis was conducted by using search terms, such as 'stroke patient eating' and 'eating after stroke'. Within the given inclusion and exclusion criteria, there were a few actual videos selected to use for analysis. Through iterative observations of those videos, the collected data and results will be presented by written summary and sketch diagram. 
The research in the thesis is conducted by critically engaging in the design process. Each iterative experiments, design process and results were subjected to critical assessment and reflection (Pedgley, 2007). The iteration of design became an essential guide to lead the development and reviews of prototypes to ensure the functional needs and requirements were met.

\subsection{Recruitment and feedback}

Recruitment of stroke patients began with a presentation of the project and with a moderator in an advanced guide. The relevant questionnaire of pathological conditions was sent to clinicians earlier before interviewing the participants.

Prior to the design research and design process, the inclusion and exclusion criteria were given to filter out the correct target group and participants to take part in this research.

The phase of participant recruitment was carried out with the help of the Stroke Foundation New Zealand and Auckland University of Technology (AUT). The participant selection criteria are as follows:

\section{Inclusion Criteria}

1. Aged $>18$

2. Has experienced a stroke which affects upper limb function.

3. Has at least 10 degrees of finger flexion. 


\section{Exclusion Criteria}

1. Significant cognitive deficit in the opinion of the screening physiotherapist.

2. Unable to follow a one-step verbal instruction.

3. Unable to give informed consent.

4. Medically unsuitable in the opinion of the screening physiotherapist, GP or medical specialist.

5. Experiences excessive joint pain

6. Suffering other conditions that could affect results (e.g. substance abuse, significant mental illness such as major depression).

Due to the strict ethical restrictions from the Health and Disabilities Ethics Committee and under guidance by neuro physiotherapists, the prototypes were required to be tested with clinicians before we could test them with stroke patients. Therefore, it is a necessary link to have the usability testing sessions with clinicians before the sessions with stroke patients.

\subsection{Stigma and usability assessment}

The prototype will undergo user testing in order to examine what works for users and what does not. The participant will fill out a revised product appraisal model for stigma (PAMS) (Vaes, 2014) questionnaire and a revised System Usability Scale (SUS) questionnaire. These questionnaires utilised a Likert 7-point scale, ranging from agreement to disagreement. The original PAMS and SUS model is in chapter 1. 


\subsection{Review session with clinicians}

Semi-structured interviews (Wood, 1997) were employed to gain a deep understanding of the problem that can reveal from interviewees. The semi-structured interviews in this research were conducted with the expert clinicians and users. The interview with Occupational Therapist (OT) and Physiotherapist (PT) helped the researcher to collect the necessary usability and background knowledge.

An initial observation session and expert review was held in AUT and a rehabilitation institution. The observation relied on a fly-on-the-wall method to observe the actual patients in order to reduce complicated emotion between participants and observers. The observation session provided general insights about stroke rehabilitation and difficulty of their conditions. After the observation in the rehabilitation institution, an opening discussion was conducted through an expert review.

The second expert review was held in Wellington while AUT clinicians came to the VUW Design School. The review was primarily presented by researchers reporting and collecting experts' feedback about different initial design concepts.

The usability testing was held at AUT with an expert review by OT and PT. The initial prototypes and current AT cutlery products were both shown to the expert clinicians in order to compare the differences of each design and collect feedback for the next design stage.

Professional insight helped expand and critically reflected upon informing the next stage of generating feasible concepts and prototypes (Shirzad et al., 2015, p. 363). When designing for a participant, especially a stroke patient in such a sensitive position, reflection and analysis remind the researcher of their responsibilities as a designer. The responsibilities include interpreting the patients with empathy and respect, and ensuring the participants are under the utmost care for their medical, physical and emotional sensitivities. The iterative design would allow me to fine tune the design to suit different patients. 
In this thesis, FDM and Polyjet technology were primarily used to create the prototypes. 3D printing technologies are substantially less limited by form complexity than many traditional manufacturing techniques. 3D printing has become a valuable tool for enabling rapid prototyping of custom designs. Within the study of cutlery handles, 3D printing has become an effective tool for testing and shape. In addition, 3D printing can provide a customisable scenario for a user who might have different demands.

Nevertheless, many elements of the iterations will be designed to be SLS printed in metal. For example, the edges of the knife and the bowl of the spoon need to be print with a precise thickness and a detailed layer solution. I believe, however, that the rapid advance in application of metallic 3D printing will become a viable option in the near future. 



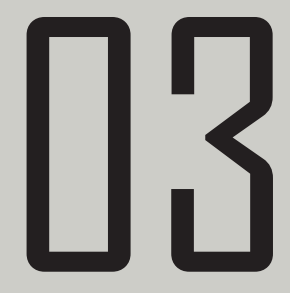

DESIGN RESEARCH 
The background research has demonstrated that there is an opportunity to develop the cutlery set. Hence, the preparation work of developing needs to be conducted. Examples include collecting clinicians' views and analysis of eating difficulties.

Disability can lead to problems in people's lives, either directly or indirectly. These problems are either viewed as being inherent in impairment itself, or as being created by the designed environment and other people's behaviors. The respective terms people with disabilities and disabled people (people disabled by the society they live within) emphasize each of these perspectives (Pullin, 2009). Many designers treat design for disability as part of engineering and human factors, and perceive disability in terms of approaching legislation that threatens to compromise their creativity, rather than as a source of fresh perspectives that could catalyze new directions and enrich the whole of their work (Pullin, 2009). 


\subsection{Observation session}

The initial discussion sessions and workshops with clinicians were conducted at the Auckland University of Technology in the occupational therapy research facility. The presentation was attended by several stroke clinicians, professors of stroke research, and physiotherapists. The presentation was followed by project introduction and opening discussion. After the presentation, there was a 10-15 minute observation session visiting a rehabilitation clinic in Auckland. The particular group mainly consisted of senior citizens who have Parkinson's and are trying to keep active mobility by continuously exercising. The physiotherapists were next to the participants in the whole exercise process, either aiding or talking with them. Most of the participants in the clinic were doing a lower limb exercise and lower limb mobility rehabilitation. Indeed, there were several upper limb exercises to help participants regain upper limb mobility. Examples include lifting a weighted ball or stretching a rubber band. However, there were no apparent hand exercises that focused on helping them regain their eating ability through the observation. Most upper limb exercises in the rehabilitation were focused on a large part of the body. In fact, the whole process did not involve an activity that helped participants eat.

After the observation, there was an opening discussion when we went back to the AUT campus. The discussion started with expert clinicians talking about their years of experience with stroke rehabilitation. The design students explained some design theories that about assistive technology product.

The opening discussion not only gave insights about what problems that patients face but also helped further establish the framework as a foundation for design.

\subsection{Discussion session with clinicians}

The second discussion session with clinicians aimed to collectively consolidate all the comments and raise patients' difficulties by their years of clinical experience. In aiming to generate the design concepts, a quick modelling by play dough helped the designer to compose design concepts. The collection also highlighted a few difficulties by writing opinions in note paper. The summary is as follows: 
- $\quad$ The shoulder weakness restricts the arm lift movement, it is difficult to turn forward the spoon and fork (Radial deviation when attempting to feed themselves.

- $\quad$ Lack of grip and grasp strength.

- $\quad$ Food falls off the cutlery.

- Wrist pain when holding an object to do a flexion motion.

- Muscle contraction on finger, except the thumb, the left four fingers are easily limited and cannot open palm neatly.

- Muscle contraction leads the grip motion stiffly.

- $\quad$ Fingers are flexed so that it is hard for cutlery handles to fit into palm.

- $\quad$ Embarrassment when using adaptive cutlery.

- $\quad$ Don't have enough strength to do a cutting motion and use knife.

- Abduct shoulder.

- $\quad$ Slow moving motion.

- $\quad$ Pain for shoulder reduced movement.

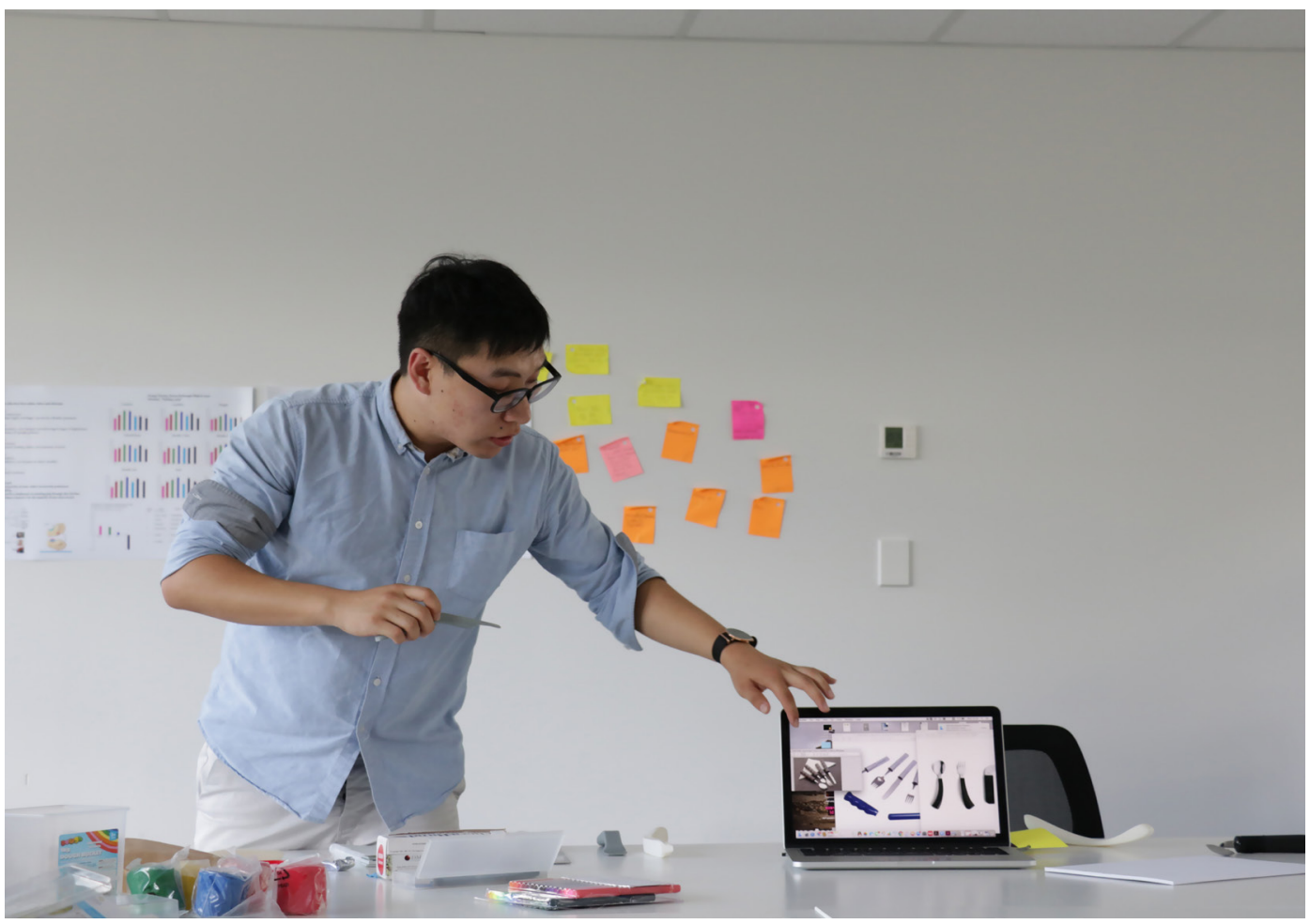

Figure 3.1 AUT discussion and feedback collection 


\subsection{Eating Video analysis}

To explore different difficulties that affect stroke patients, there was a systematical video analysis through online research. The database used Google online video search.

The first search term was 'eating after stroke', and 523000 results showed in the video list. The second search term was 'stroke patient eating', and 561000 results showed in the video list. The third search term was 'stroke feeding', and 233000 results showed in the video list. The fourth search term was 'living with stroke', and 780,000 results showed in the video list. The fifth search term was 'life after stroke', and 574000 results showed in the video list. The sixth search item was 'eating and stroke', and 636000 results showed in the video list. The seventh search item was 'constraint induced therapy eating', and 17400 results showed in the video list. The date of selected videos ranged from 2000 to 2017 . Before selecting the video, the patient needed to follow the given inclusion and exclusion criteria described in Chapter 2. The inclusion and exclusion criteria for selecting videos are as follows:

Inclusion Criteria:

- The video clearly displays the upper limb and holding cutlery in eating.

- Had experienced stroke which impacts on upper limb function.

- The person in the video is doing an eating activity.

- The person in the video has difficulty in eating, but they can use the cutlery to feed themselves.

- The eating video was filmed in a real physical environment, and the patient in the video is a real patient.

- The video should be an objective pattern to record patient eating.

Exclusion Criteria

- The patient cannot feed themselves, and they have assistance from others.

- The video is not recording a real eating activity, and the patient in the video is not a real stroke patient.

- The patient in the video did not use their affected upper limb to hold the cutlery.

- The patient in the video has no mobility to move his/her upper limb. 
The video analyses were generated in response to the background research and inform a developed stage of criteria. The videos passed through screening were decided by the given inclusion and exclusion criteria.

In the process of video analysis, the collection and notes were taken in response to patients' challenge of upper limb accomplishing eating tasks. The main results are summarized as follows:

1. The forearm is hard to pronate.

2. The shoulder is painful when trying to lift the affected arm.

3. The impaired shoulder makes the arm and forearm lift with difficulty.

4. Middle finger, ring finger (third finger) and pinkie are difficult to open because of muscle contraction.

5. The index finger may keep a temporary bending feature when the patients are trying grip an object.

6. Some chronic stroke patients or elderly patients may have spasticity, which makes them have a patulous palm feature.

7. Middle finger, ring finger and pinkie are too weak to grip and hold an object.

8. Index finger and thumb are the main force drivers to hold an object, and their holding strength is limited by stroke impairment.

To summary the findings, the difficulty issues of holding cutlery are as follows:

Difficulty in bending wrist towards body; tilts hand to side away from body.

Difficulty in bending finger to make a fist.

Difficulty in stabilising wrist.

Difficulty in opening fingers and grasping the utensil handle.

Difficulty in bending tip of thumb; extending thumb.

Difficulty in bending index finger; extension and flexion.

Difficulty in moving thumbs up and down when holding the handle side. 
To define paramters of how hands are affected, the design research explores common patterns that appeared in patients' upper limb. The summary is as follows:

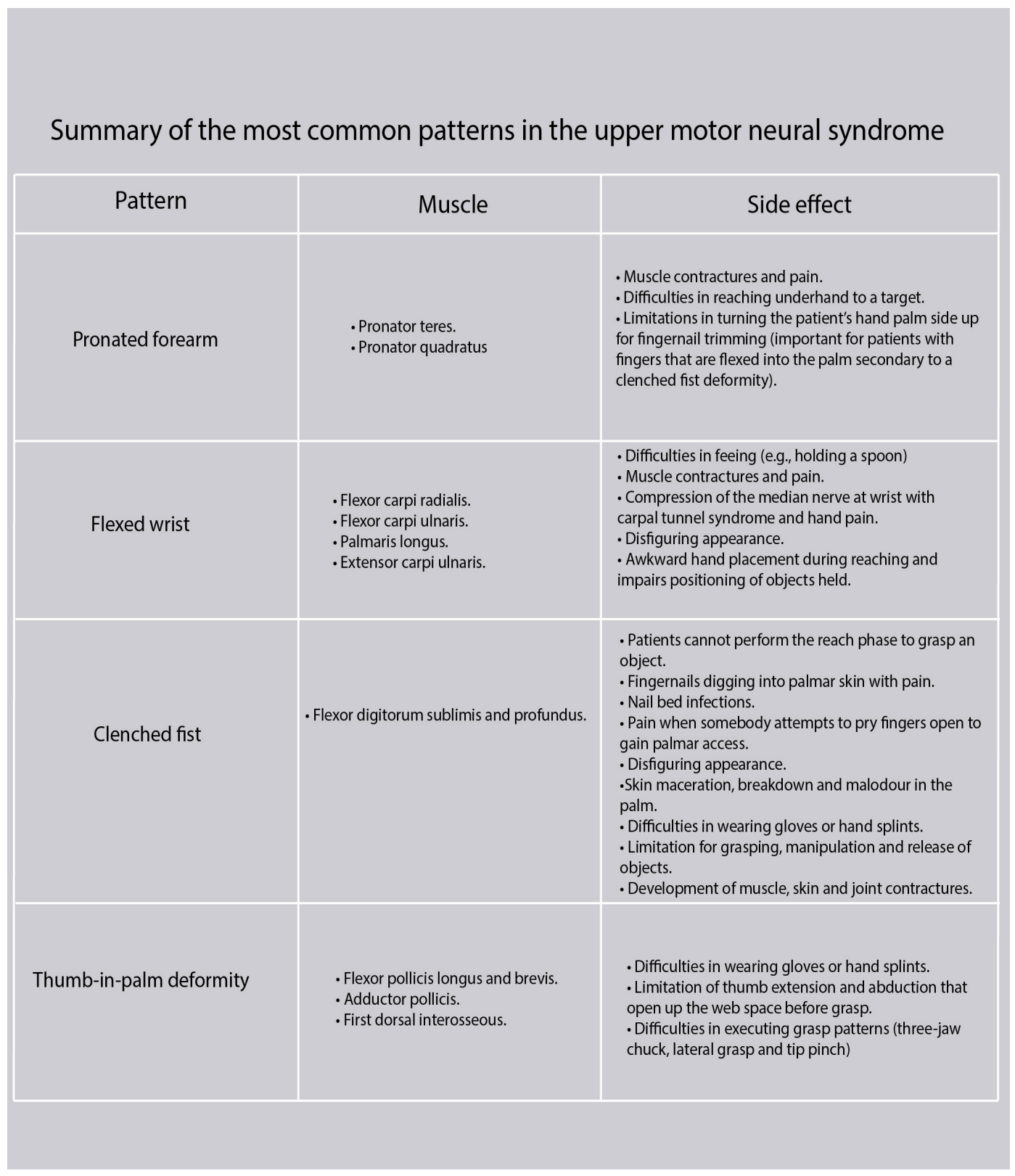

Figure 3.2 Texting support by Thibaut (2013). 
Stroke patient arm kinematic analysis
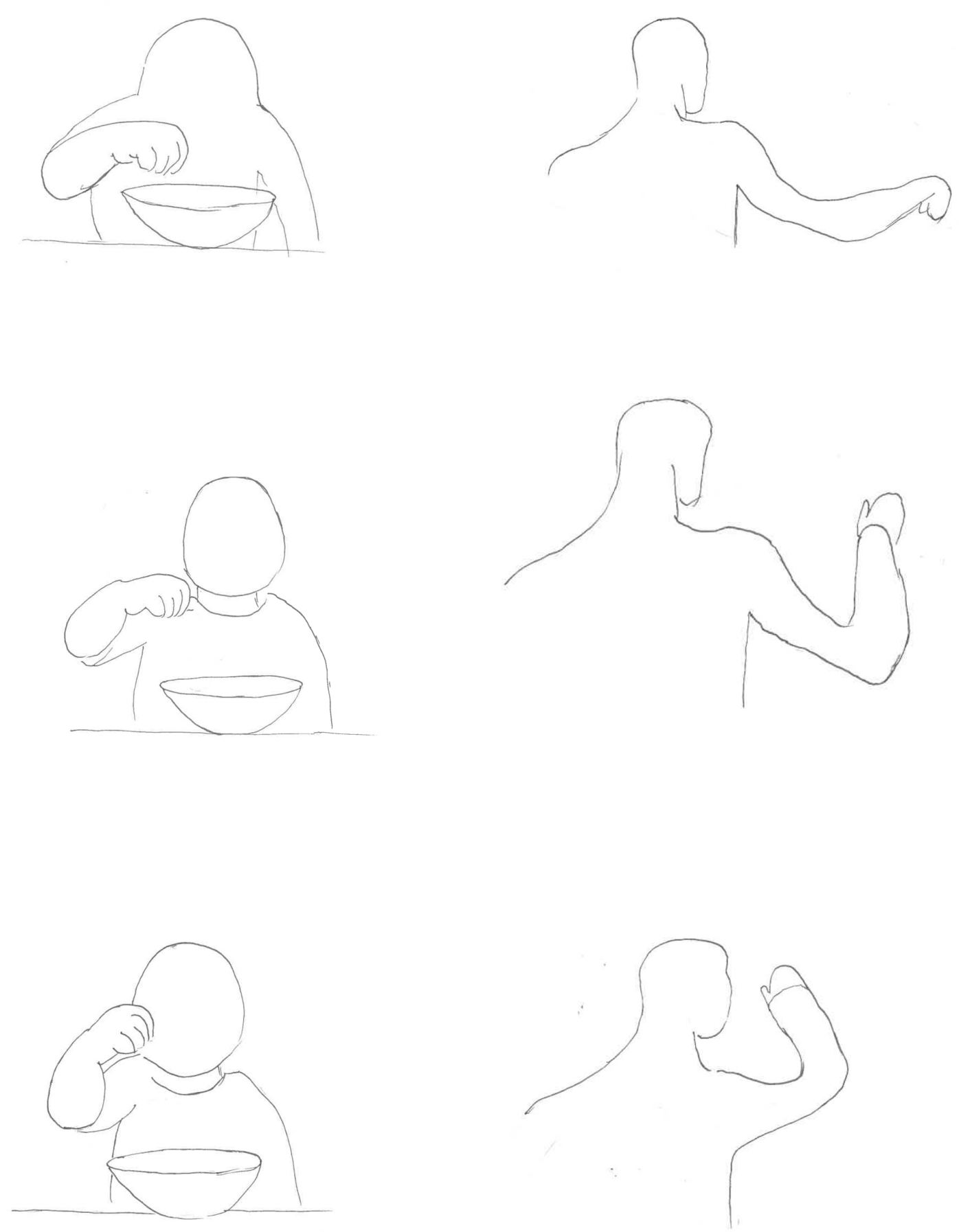


\section{(People dont have stroke) arm kinematic in eating}
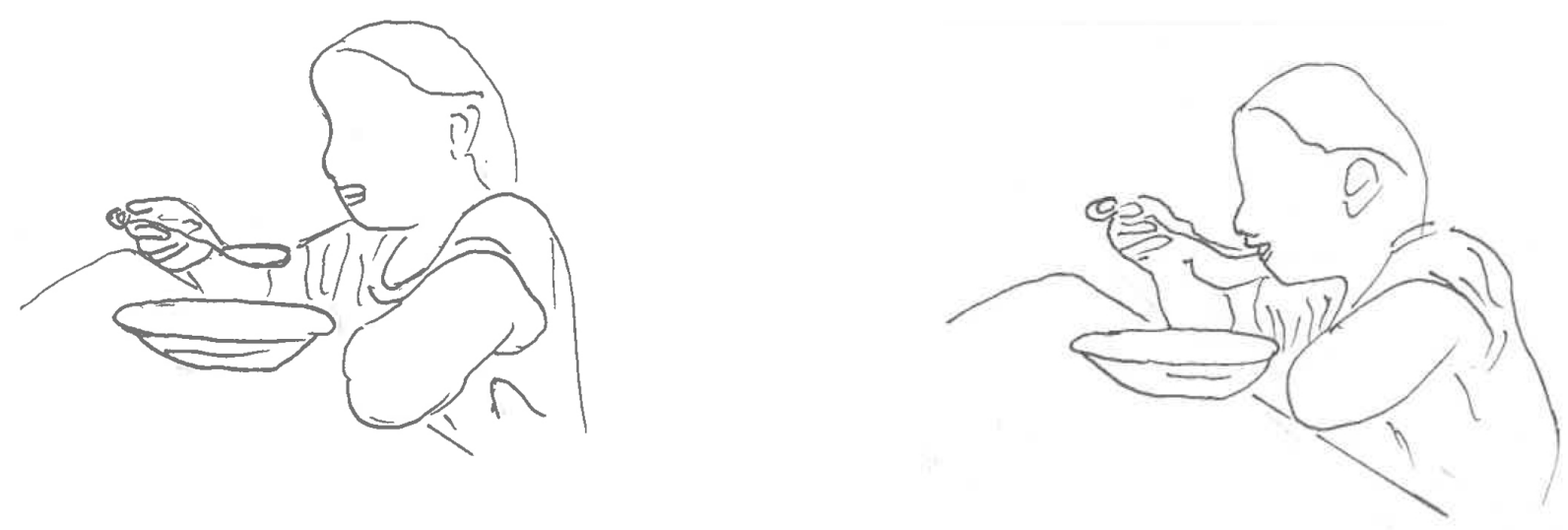

(Stroke patient) arm kinematic in eating
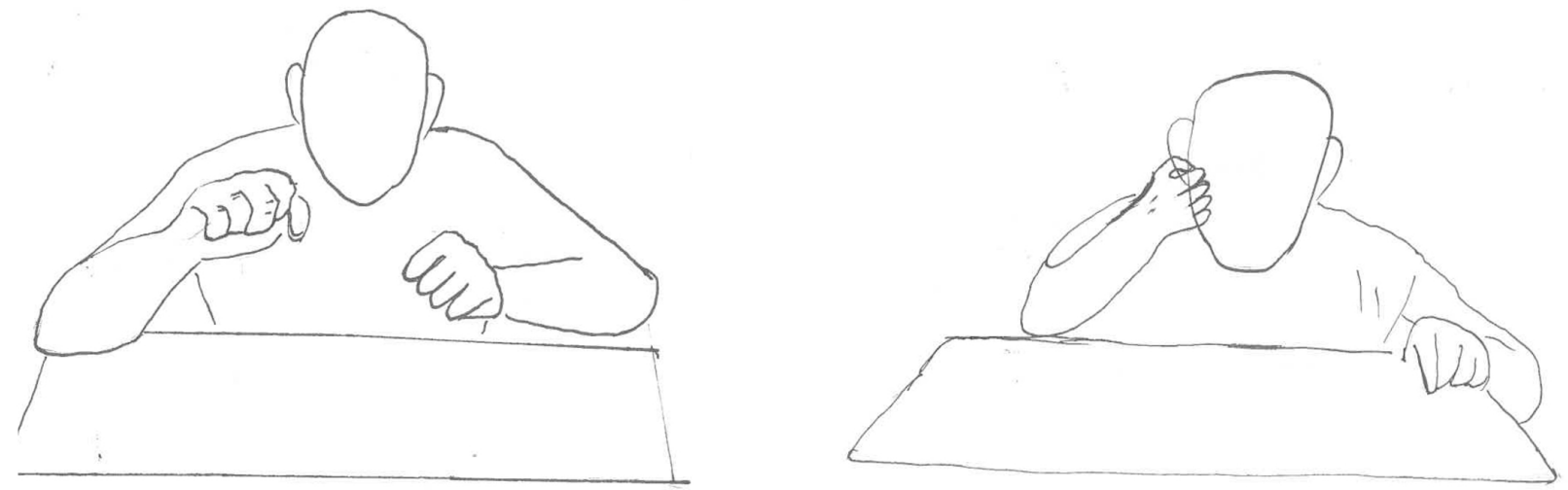

Figure 3.3 Through analysing stroke patient eating movement, the arm kinematic movement is described by the sketches. 


\subsection{Defining Design intervention}

A successful stigma-free product is often the result of several complementing design interventions.

The original intervention models of Vaes (2014) comprise a set of 17 design interventions that enable designers to alleviate products of sigma eliciting properties. By incorporating these interventions, designers can aim for solutions that relieve product-users from the social stress related to owning or using a protective, assistive or medical device in unwelcome contexts. Apart from the benefits for the individual user, additional benefits can include increased user-product attachment and collective well-being (Vaes, 2014, p.172).

The reason for choosing the Product Intervention Model for Stigma (PIMS) is because it is specifically intended to meet the stigma relevant design requirements that arose during the application of the PAMS.

As background research suggested earlier, assistive devices are often rejected by users for some reasons such as their technical function or a lack of skill or confidence in using the product.

In response to identifying the target group stroke patients and opportunity of design, the chosen and original interventions are as follows: 


\section{Selected design interventions from PIMS}

\begin{tabular}{|c|c|c|}
\hline Intervention & Description & Informed design criteria \\
\hline $\begin{array}{l}\text { Can the designer camouflage or disguise } \\
\text { stigma-sensitive product features? }\end{array}$ & $\begin{array}{l}\text { The aim of this intervention is to camouflage or disguise the } \\
\text { entire product or its most prominent stigma-sensitive } \\
\text { features. It challenges designers to conceive products that } \\
\text { make the user happy without feelings of emotional } \\
\text { attachment. The use of translucent or skin-coloured } \\
\text { materials to hide the obtrusiveness of certain design } \\
\text { features is an example of this intervention. Taken to its } \\
\text { extreme, this intervention should render a product invisible. }\end{array}$ & $\begin{array}{l}\text { An aesthetic appearance design will camouflage or } \\
\text { disguise stigma-sensitive features. }\end{array}$ \\
\hline $\begin{array}{l}\text { Can the designer divert the bystander's } \\
\text { attention away from stigma-sensitive } \\
\text { product features? }\end{array}$ & $\begin{array}{l}\text { This intervention suggests that the designer should aim for } \\
\text { a diversion of the bystander's attention, away from the } \\
\text { stigma-sensitive features, towards more appealing or } \\
\text { eye-catching features. The bystander's attention can also } \\
\text { be lured away from the stigma-sensitive product as a } \\
\text { whole, towards a more accepted product within the visual } \\
\text { scope of the bystander. }\end{array}$ & $\begin{array}{l}\text { The design should not attract a bystander's negative } \\
\text { attention to the users. }\end{array}$ \\
\hline $\begin{array}{l}\text { Can the designer strengthen the product's } \\
\text { individual identity? }\end{array}$ & $\begin{array}{l}\text { In this intervention the designer aims to reshape the } \\
\text { product in such a way that users wish to associate } \\
\text { themselves with that product, and possibly value it as an } \\
\text { extension of or addition to their personality. }\end{array}$ & $\begin{array}{l}\text { The design should increase the identity through the } \\
\text { design quality. }\end{array}$ \\
\hline $\begin{array}{l}\text { Can the designer eliminate physically or } \\
\text { mentally confronting moments in product } \\
\text { use? }\end{array}$ & $\begin{array}{l}\text { In this intervention the designer adapts the product's } \\
\text { functionality or its usage rituals in such a way that } \\
\text { physically or mentally confronting moments in product use } \\
\text { can be avoided. }\end{array}$ & $\begin{array}{l}\text { The design should increase the usability and } \\
\text { ergonomics in user experience. }\end{array}$ \\
\hline $\begin{array}{l}\text { Can the designer manage the frequency } \\
\text { and intensity of product use? }\end{array}$ & $\begin{array}{l}\text { In this intervention the designer aims to adapt the product } \\
\text { in such a way that users can limit the frequency or intensity } \\
\text { of product use in order to reduce social tension and } \\
\text { exposure. }\end{array}$ & $\begin{array}{l}\text { The design should increase the user's interest so they } \\
\text { are happy to use it. }\end{array}$ \\
\hline $\begin{array}{l}\text { Can the designer limit PRS by focusing on } \\
\text { the ultimate product goals? }\end{array}$ & $\begin{array}{l}\text { In this intervention the designer places an extreme focus } \\
\text { on the actual or ultimate product goal. By exclusively } \\
\text { addressing this ultimate goal, the product could become } \\
\text { obsolete in all other situations. }\end{array}$ & $\begin{array}{l}\text { The design should address stigma with the PIMS } \\
\text { model. }\end{array}$ \\
\hline $\begin{array}{l}\text { Can the designer reshape product meaning } \\
\text { through advances in technology? }\end{array}$ & $\begin{array}{l}\text { In this intervention the designer aims to reshape the } \\
\text { meaning of the product by applying new technology. } \\
\text { Applying new technology can make a product smaller, more } \\
\text { performing, and cheaper to buy and own. }\end{array}$ & $\begin{array}{l}\text { The 3D printing technology should present its } \\
\text { advances in a customizable design scenario. }\end{array}$ \\
\hline $\begin{array}{l}\text { Can the designer reshape the product } \\
\text { meaning through advances in material } \\
\text { technology? }\end{array}$ & $\begin{array}{l}\text { In this intervention the designer aims to reshape the } \\
\text { meaning of the product by applying new materials or } \\
\text { material technology. Implementing new materials can } \\
\text { make a product lighter, stronger, more ecological, cheaper } \\
\text { and more widely accessible to users. }\end{array}$ & $\begin{array}{l}\text { The 3D printing technology should present its } \\
\text { advances in a customizable design scenario. }\end{array}$ \\
\hline $\begin{array}{l}\text { Can the designer reshape product meaning } \\
\text { through the product's meaningful } \\
\text { interaction with other products? }\end{array}$ & $\begin{array}{l}\text { In this intervention the designer aims to reshape the } \\
\text { meaning of the product by reflecting on its possible } \\
\text { interaction with other products. The designers can } \\
\text { generate a product that mimics the typology of a product } \\
\text { that is accepted or even celebrated. }\end{array}$ & $\begin{array}{l}\text { The design should be accepted by interacting with } \\
\text { other products. }\end{array}$ \\
\hline $\begin{array}{l}\text { Can the designer endow the product user } \\
\text { with extra abilities? }\end{array}$ & $\begin{array}{l}\text { In this intervention the designer aims to develop a product } \\
\text { that will endow the product user with extra abilities. Extra } \\
\text { ability can also be suggested. }\end{array}$ & $\begin{array}{l}\text { The design should endow and increase the user's } \\
\text { confidence when using it in front of bystanders. }\end{array}$ \\
\hline $\begin{array}{l}\text { Can the designer boost the user's social } \\
\text { skills? }\end{array}$ & $\begin{array}{l}\text { In this intervention the designer aims to reshape the } \\
\text { meaning of the product so that it delivers a boost to the } \\
\text { user's social skills. This intervention can make the user rise } \\
\text { above the reactions of others by making him or her visually } \\
\text { or verbally more assertive. }\end{array}$ & $\begin{array}{l}\text { The design should enable the user confidently } \\
\text { introduce it to bystanders. }\end{array}$ \\
\hline
\end{tabular}




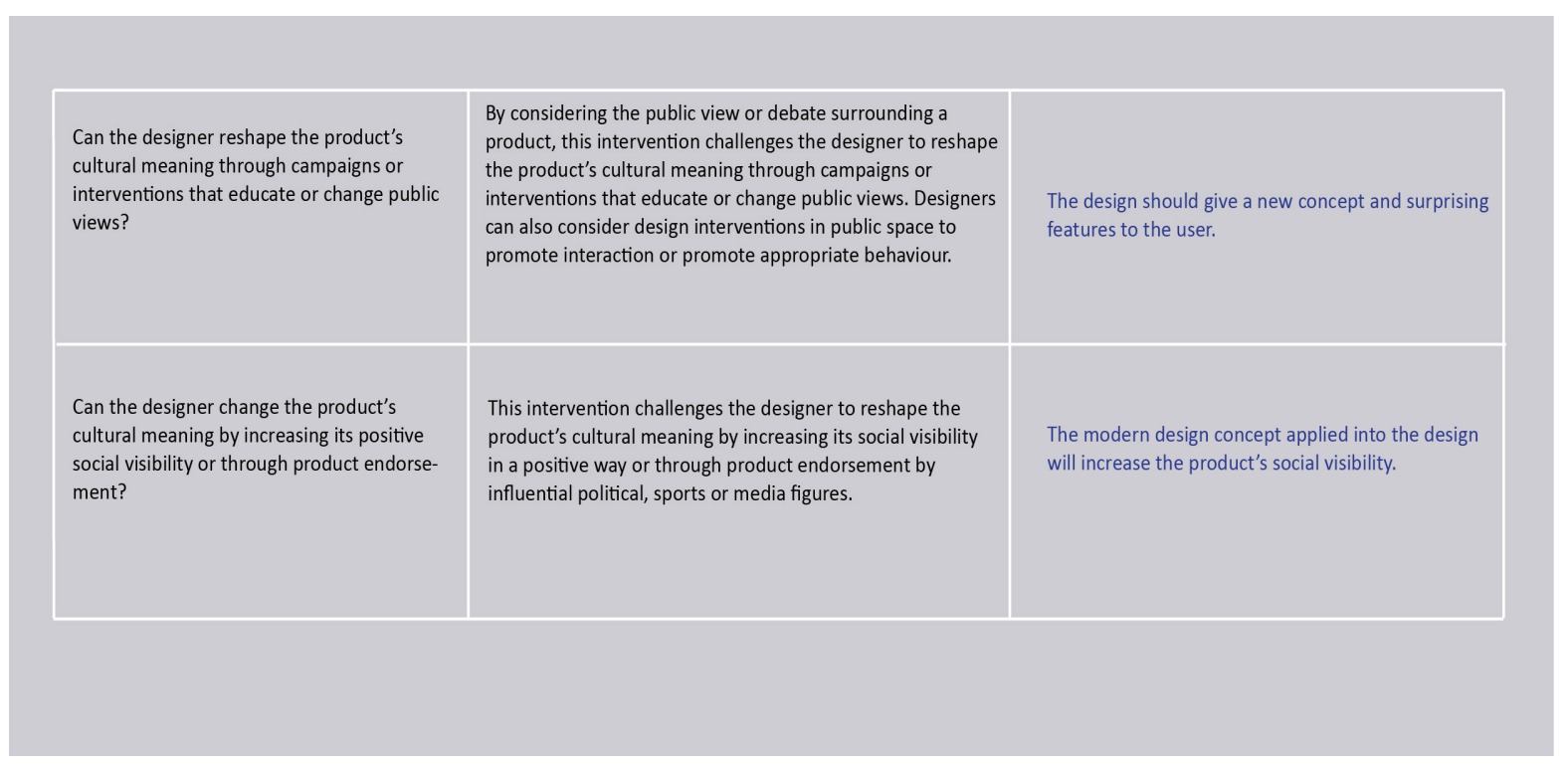

Figure 3.4 Texting support by Vaes (2014). 


\subsection{Final design criteria}

Function

- The cutlery set must adapt to patients at different ability levels. This would help patients to continuously use the product.

- The design should adapt to different stages of stroke. (Within the inclusion criteria)

\section{Aesthetic}

The user should interact with the utensils and uses the devices should be informed through clear visual cues.

- The aesthetic of the design should reflect its usability through the interaction.

Addressing stigma associated with medical products: In terms of the PAMS (Vaes, 2014) in this design, designing stigma-free utensils is one of the primary aims in this study. The users do not want their utensils looking like a strange design, or similar to a medical device. The aesthetic of the product should be designed to be used in the home and public restaurants, so there will be no association of the user being sick or disabled.

- The design should look like an elegant cutlery product.

- The design should encourage the user to use it confidently.

- The design should elicit pride and avoid embarrassment.

These criteria are the foundation of the design framework. The framework of the design concepts, together with the feedback from clinicians, will allow the rapid generation and prototypes process. Each iteration of the design was informed by the experiment and reviews from clinicians. The final design concepts will be a result of participant testing sessions . 


\begin{tabular}{|c|c|c|c|}
\hline & Information & Value & Affordance \\
\hline \multicolumn{4}{|c|}{ Aspects related to product stimulus components } \\
\hline $\begin{array}{l}\text { Product perspectives/ } \\
\text { Differentiations }\end{array}$ & $\begin{array}{l}\text { Aesthetics } \\
\text { How does the product } \\
\text { look \& feel? }\end{array}$ & $\begin{array}{l}\text { Function } \\
\text { What is the product } \\
\text { purpose? Product } \\
\text { benefits? }\end{array}$ & $\begin{array}{l}\text { Interaction } \\
\text { How does the user } \\
\text { interact with the } \\
\text { product? }\end{array}$ \\
\hline Product stimulus levels & Perception & Cognition & Cognition \\
\hline $\begin{array}{l}\text { Behavioral sequences } \\
\text { Krippendorff (2006) }\end{array}$ & Sensing & Meaning & Acting \\
\hline $\begin{array}{l}\text { Appraisal in relation } \\
\text { to product stimulus } \\
\text { components (Desmet, } \\
\text { 2002) }\end{array}$ & $\begin{array}{l}\text { Non instrumental } \\
\text { interaction } \\
\text { component } \\
\text { (perceptions) }\end{array}$ & $\begin{array}{l}\text { Non-physical } \\
\text { interaction } \\
\text { component } \\
\text { (consequences) }\end{array}$ & $\begin{array}{l}\text { Instrumental } \\
\text { interaction } \\
\text { component (use) }\end{array}$ \\
\hline \multicolumn{4}{|c|}{ Aspects related to human concerns } \\
\hline $\begin{array}{l}\text { Types of product } \\
\text { benefits } \\
\text { (Jordan, 2000) }\end{array}$ & $\begin{array}{l}\text { Hedonic benefits } \\
\text { Sensory and aesthetic } \\
\text { pleasures }\end{array}$ & $\begin{array}{l}\text { Emotional benefits } \\
\text { Effects on user's } \\
\text { emotions }\end{array}$ & $\begin{array}{l}\text { Practical benefits } \\
\text { Result from the } \\
\text { completion of tasks }\end{array}$ \\
\hline $\begin{array}{l}\text { Apppraisal in relatión } \\
\text { to human concerns } \\
\text { (Desmet, 2002) }\end{array}$ & $\begin{array}{l}\text { Sêrisory perception } \\
\text { appraisal in view of } \\
\text { human attitudes }\end{array}$ & $\begin{array}{l}\text { Cognitive } \\
\text { interpretation } \\
\text { appraisal in view of } \\
\text { human standards }\end{array}$ & $\begin{array}{l}\text { Whysical action } \\
\text { appraisal in view of } \\
\text { goals }\end{array}$ \\
\hline $\begin{array}{l}\text { Design Goals } \\
\text { (Sanders, 1992) }\end{array}$ & $\begin{array}{l}\text { Desirable } \\
\text { Aesthetically appealing }\end{array}$ & $\begin{array}{l}\text { Usable } \\
\text { Capacity to be } \\
\text { understood, learned \& } \\
\text { utilized }\end{array}$ & $\begin{array}{l}\text { Useful } \\
\text { Accomplishes what it } \\
\text { was designed for }\end{array}$ \\
\hline $\begin{array}{l}\text { Levels of } \\
\text { Processing } \\
\text { (Norman, 2004) }\end{array}$ & $\begin{array}{l}\text { Visceral } \\
\text { Aesthetic and tactile } \\
\text { qualities }\end{array}$ & $\begin{array}{l}\text { Reflective } \\
\text { Self-image, personal } \\
\text { satisfaction, memories }\end{array}$ & $\begin{array}{l}\text { Behavioral } \\
\text { Effectiveness and ease } \\
\text { of use }\end{array}$ \\
\hline
\end{tabular}

Figure 3.5 'Similarities between different design models that point to complementing ideas on how to implement affect and emotion in product design' (Vaes, 2014, p.28). 



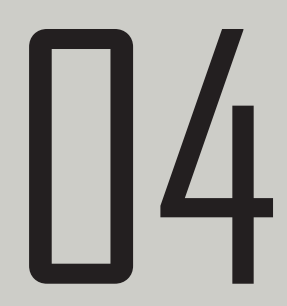

DESIGN PRDCESS 
Cutlery design evolving in history has been shown a unique characteristic in which its minor features are the key factor. Through the historical analysis of cutlery evolution, the progress of cutlery from rough to complicated handle decoration, and to simple streamline trends also reflect users' demands and improvements. Vaes (2014) indicates that the cultural and domestic eating background will influence user selection options. Therefore, we should consider the user's lifestyle and eating habits when designing stigma-free cutlery. In this chapter, I will break down several minor features of cutlery in order to maximize aesthetic considerations.

\section{$4.12 D \& 3 D$ framework}

The earlier stage of design starts from 2D and 3D sketches. A sketch presents a very basic way in which the physical factors, such as crumpling details, can be visualized and analyzed prior to computer software modelling. The renders provide necessary visual feedback after the digital model is completed. At times the physical iterations and design concepts were presented to peers and research supervisors to obtain feedback over a period.
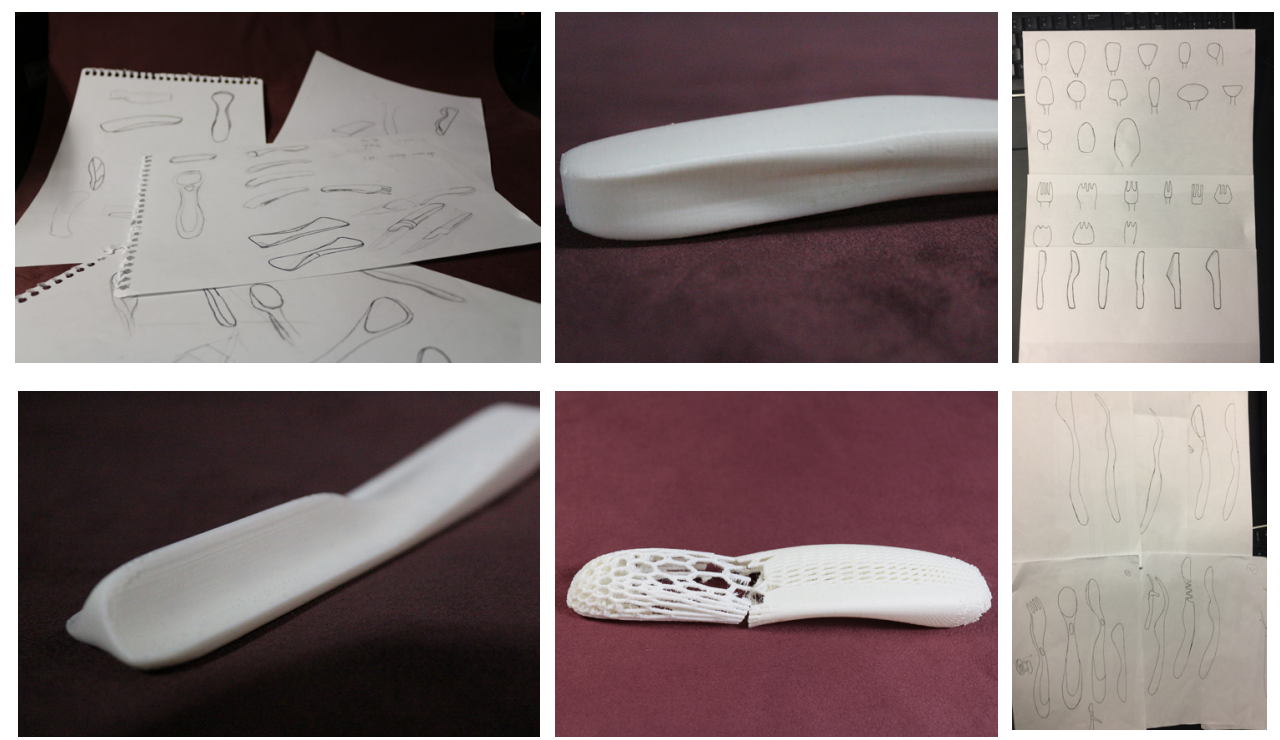

Figure 4.1 2D \& 3D Sketches, earlier stage handle model 


\subsection{The initial assumption of the handle design}

\begin{tabular}{|c|c|}
\hline Assumption & Aim \\
\hline $\begin{array}{l}\text { A layer of elastomeric material disposed on at least a portion } \\
\text { of the rigid core. }\end{array}$ & $\begin{array}{l}\text { For the purpose of solving people who have a grip weakness, } \\
\text { elastomeric material would able to increase the friction with } \\
\text { hand skin. }\end{array}$ \\
\hline A body portion extending longitudinally. & $\begin{array}{l}\text { The inclusion criteria has clarified the user will be over } 18 \\
\text { years old, so the handle should not be too short. }\end{array}$ \\
\hline $\begin{array}{l}\text { The body portion should look symmetrically in the front } \\
\text { view. }\end{array}$ & $\begin{array}{l}\text { Symmetry and balance are the key features that represent an } \\
\text { aesthetic value. }\end{array}$ \\
\hline A back end opposite the front end & knife takes another view point \\
\hline An upper surface and a lower surface. & $\begin{array}{l}\text { A smooth handle and surface would please users rather than } \\
\text { use a sticky rubber to reduce the user experience }\end{array}$ \\
\hline $\begin{array}{l}\text { An upper front gripping area should have a multitude of } \\
\text { anti-slip textures protruding from the upper surface, the } \\
\text { upper front gripping area be formed as a single-unit } \\
\text { structure with elastomeric material and be centred on a } \\
\text { point located at some distance from the front end of the } \\
\text { head portion }\end{array}$ & $\begin{array}{l}\text { At the table and most of the time, knife and fork are used to be } \\
\text { combined tools interacting with both of the user's hands.. } \\
\text { Therefore, two hands are required to be use in an eating task. } \\
\text { Because of the limited motor control patients may have in } \\
\text { their affected palm and fingers, it was hypothesized that one } \\
\text { of the affected hands would need to use a knife or fork. Due to } \\
\text { the unitive design visual language requirement, the handle is } \\
\text { streamlined to fit both hand palms }\end{array}$ \\
\hline $\begin{array}{l}\text { A lower back gripping area should have a multitude of anti-slip } \\
\text { textures protruding from the lower surface. } \\
\text { The upper front gripping area of the cutlery handle should } \\
\text { have a shape adapted to receive an index finger tip. }\end{array}$ & $\begin{array}{l}\text { By designing the stigma-free utensils that functions as assistive } \\
\text { cutlery, it was speculated that the familiarity of a cutlery form } \\
\text { would help to contribute to how it should be intuitively } \\
\text { gripped. By replicating the aesthetics of contemporary cutlery, } \\
\text { it was assumed that the design could blend into the contextual } \\
\text { eating environment and avoid any embarrassment or stigma of } \\
\text { using an assistive device. }\end{array}$ \\
\hline $\begin{array}{l}\text { The anti-slip texture in the upper front grip area is approxi- } \\
\text { mately } 2-3 \mathrm{~cm} \text { in length, and } 1.5 \mathrm{~cm} \text { in width. }\end{array}$ & $\begin{array}{l}\text { Anti-slip was considered to help people with poor grip. Most } \\
\text { anti-slip handle products adopt a single rubber material or } \\
\text { rubber material with texture on the surface }\end{array}$ \\
\hline $\begin{array}{l}\text { The design should have an inward curve between the head } \\
\text { portion and the back portion, and the curve should fit the } \\
\text { closed space of index finger and thumb. }\end{array}$ & $\begin{array}{l}\text { The research in to hand-holding cutlery presents a clear } \\
\text { image of how a hand holds the cutlery; the design should fit } \\
\text { the shape of closed palm }\end{array}$ \\
\hline $\begin{array}{l}\text { An inward curved shape in the front double side should fit the } \\
\text { thumb prominence }\end{array}$ & $\begin{array}{l}\text { A depressed area would enable user's thumb to fit and feel } \\
\text { comfortable with the handle }\end{array}$ \\
\hline
\end{tabular}

Figure 4.2 Initial assumption 


\subsection{Handle shape}

The review of current assistive technology (AT) utensils in Chapter 5 has shown a general image that most AT utensils are designed with a thick and wide handle. Initial handles were looking at a shape that could fit a clenched fist, and the paralyzed palm. The paralyzed palm causes a patulous palm, which is an opposite feature with clenched fists. The opened palm requires a thick handle of cutlery to increase a contact area with palm. In other hand, the clenched fist requires a thinner and curve shape to fit the palm. To complete this challenge, the researcher started to find a neutral solution to solve this challenge by stimulating an affected hand to hold the cutlery. Torrens (2013) indicates that the conventional shape of usual cutlery would be more psychologically acceptable for patients who have one-handed impairments. In the meantime, the streamlined handle would be willingly seen and used in eating.

The handle shape focuses on hand ergonomics and caters to user preferences of the holding feeling. The first stage of handle prototyping was conducted with clay modelling, sketches, quick digital modelling and design precedent review. The second stage of is iteratively 3D print handle prototypes.
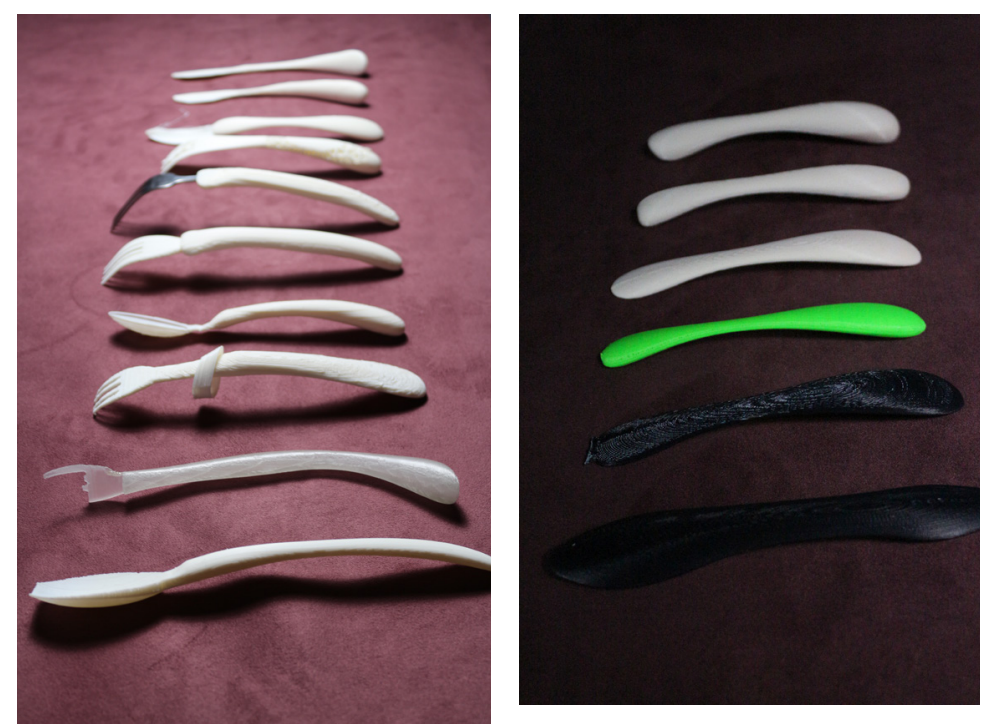

Figure 4.3 cutlery Handle prototype iterations. 
The first metal test spoon was printed in a company located in the US. The body frame was designed by an integrated frame and print in stainless steel material. However, the quality and visual aspects in this printing required a few improvements to accomplish further work.
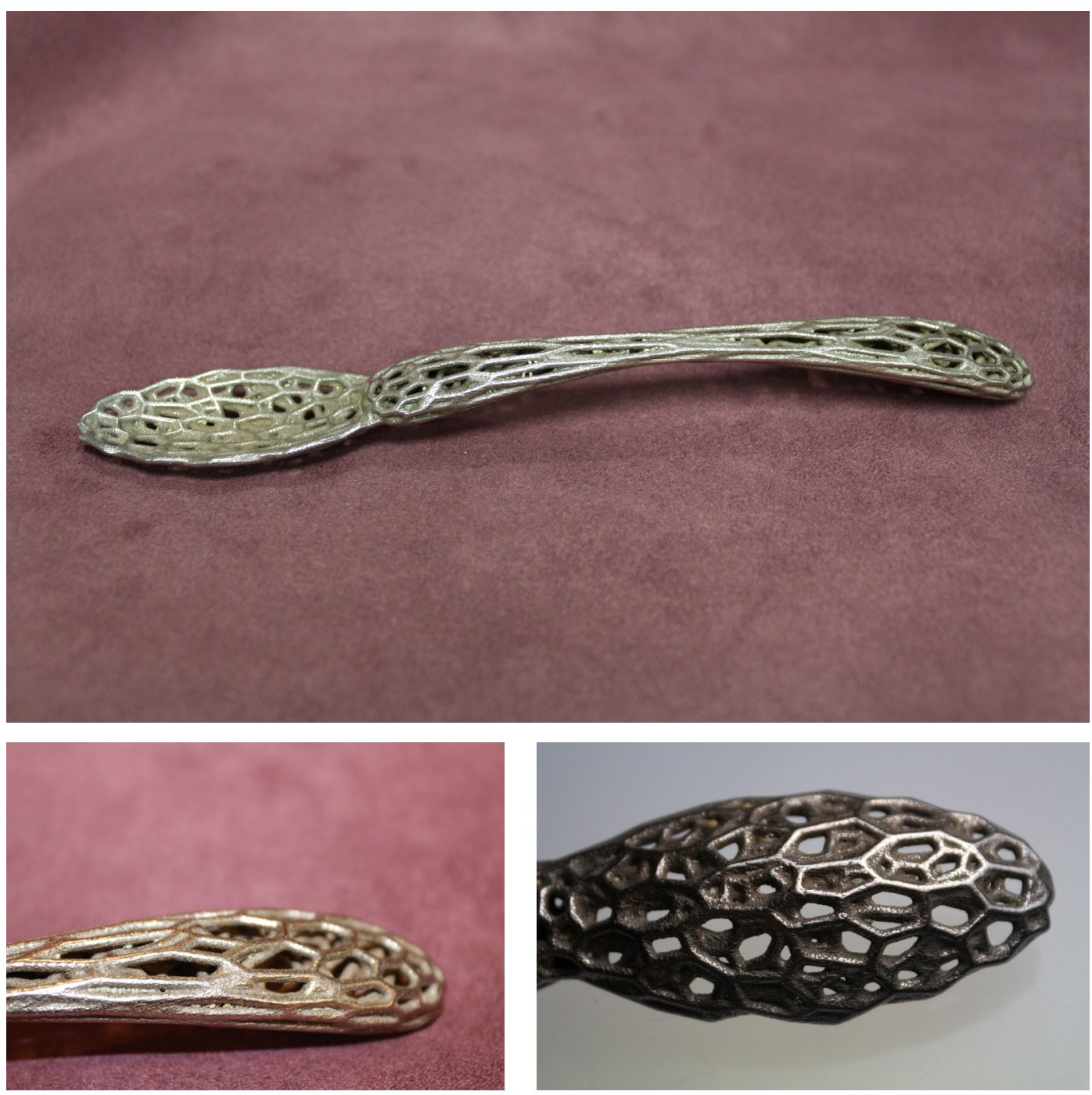

Figure 4.4 3D metal print spoon with stainless steel material 


\subsection{Developed handle design concepts}

The set of cutlery intends to achieve several criteria that developed in the last phase, such as:

1. Each unit in the set should be design with a similar visual language.

2. The handle should have a streamlined overall form.

3. Reduce the sticky material from the form.

4. A pleasurable and aesthetic appearance.

The proposed handle design followed the review of background research, intended to draw an upward curved angle along the front to the end portion. The wrist spasticity affects patient's' strength and the inability obstruct users' wrists in cutting and scooping. An upward curve in the handle would allow the user to use less strength but also accomplish their task. In the later design phase, the handle design will follow this curve criterion to continuous design.

The design was intended to be intuitive and easy to use, then the handle design would need to be easy to grasp and streamlined as well as making people feel pleased with it. Initial prototypes of the handle were focused on providing design solutions that would enable stroke patients to use them as easily as regular cutlery.
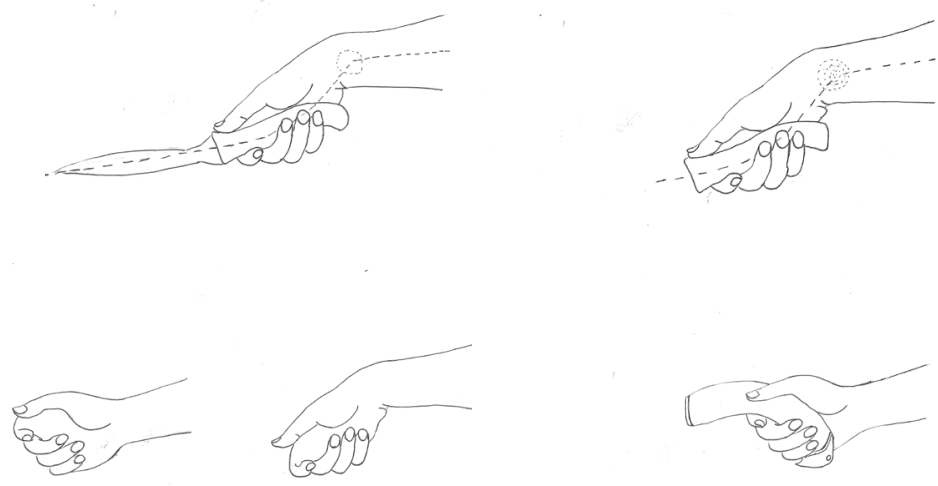

Figure 4.5 curve handle sketch 


\subsection{Clinician feedback}

When bringing a collection of current AT cutlery sets to clinicians, they indicated that the rubber and elastomeric material felt too sticky to the hand sense. Thus, in order to meet the anti-slip goal and aesthetic requirement, an integrated handle frame and smooth handle should be considered in the second stage of prototyping. Within the interview session with physiotherapists and clinicians in Auckland, they recommended that it is better to develop another attached design mainly in the front portion of the fork. The clinicians indicated that the index finger is flexed and has a weakness in pressing the fork when trying to pick up food.

A finger-wear was proposed for the affected index finger in order to stabilize the finger flexion and extension. Initial ideal finger-wear was considered to be like a jewellery ring when attempting to reach the stigma-free goal. However, the space between the rings that must allow the index finger to accomplish the flexion and extension limits the appearance of the ring.

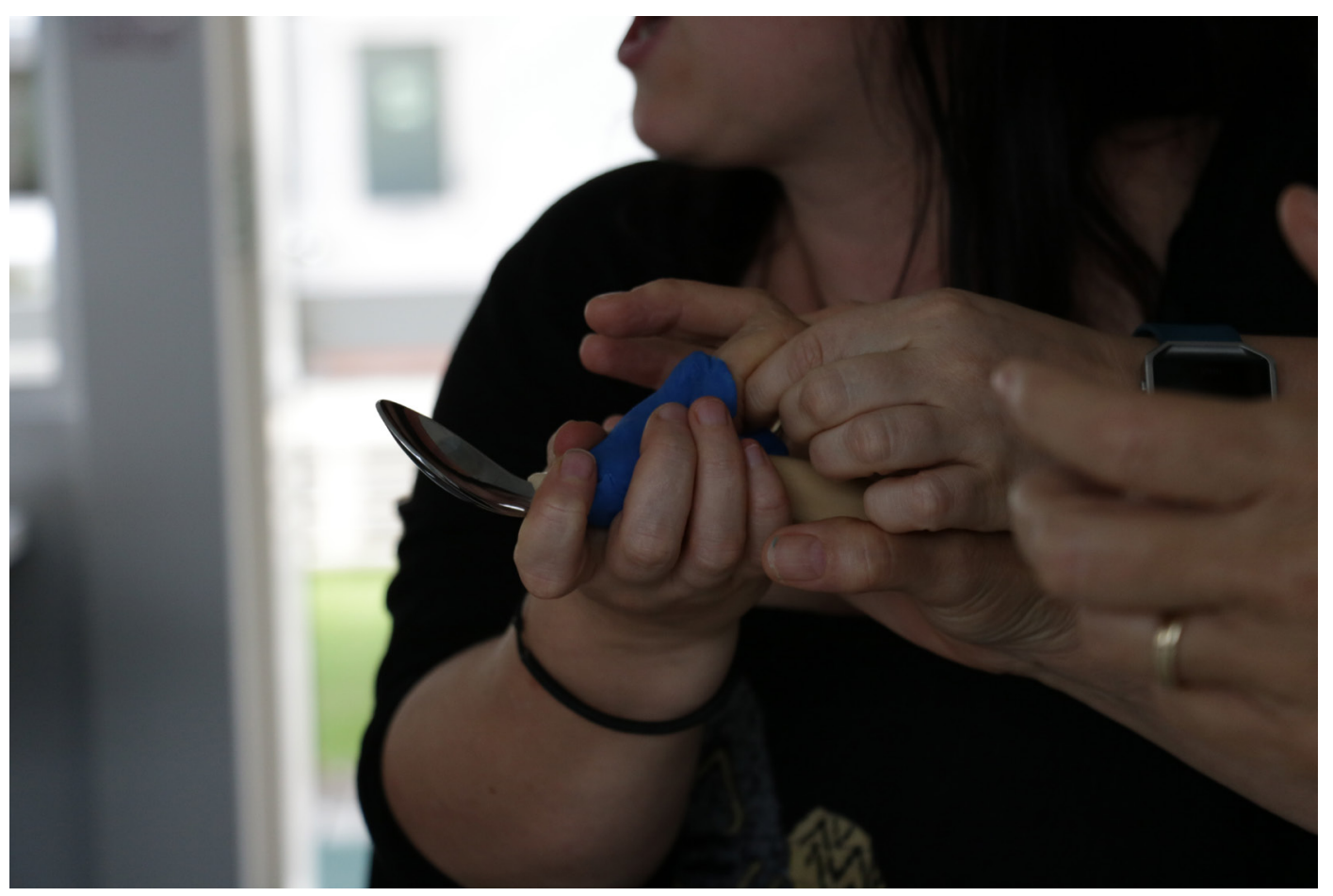

Figure 4.6 Plasticine concept model of finger wear 

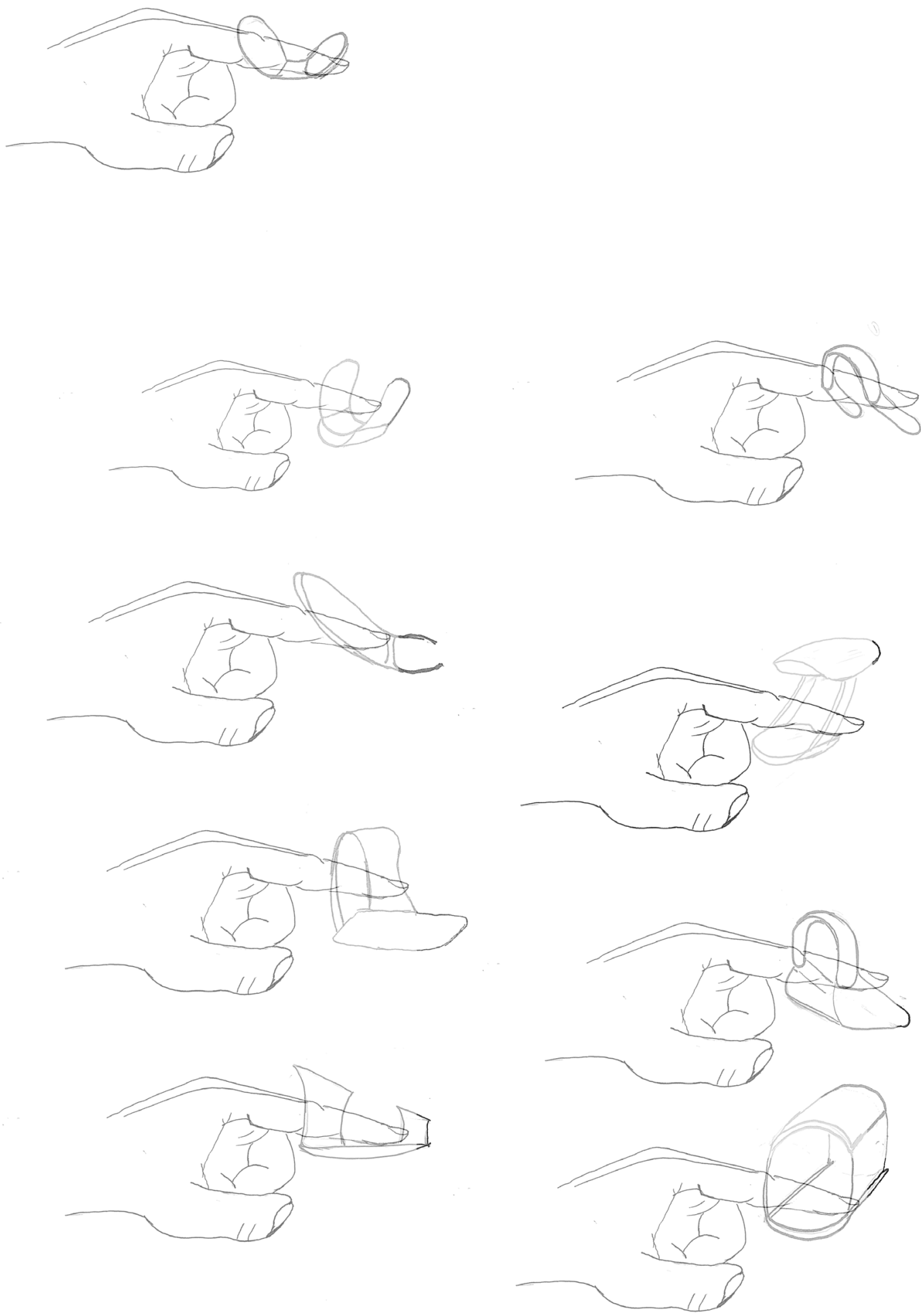

Figure 4.7 Sketches of the finger-wear concept during design process, 

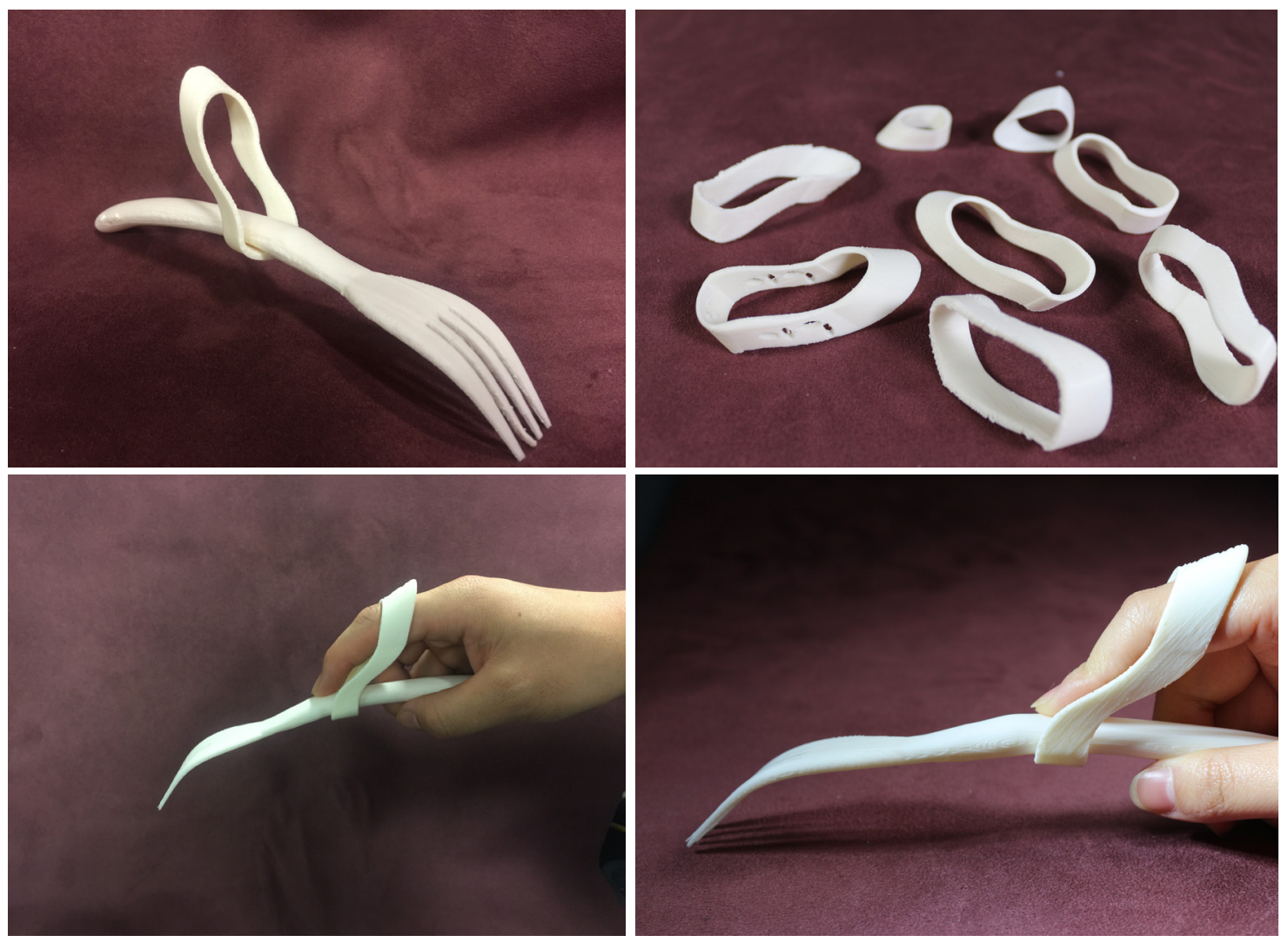

Figure 4.8 Finger-wear prototype iteration and test with mixed gender's hand. 


\subsection{Interact positions analysis and pattern}

Identifying and understanding the grip posture of the cutlery in the eating process is one of the most important factors that influence a good fit for the hand.

The eating video was carried out to help the designer understand and to inform the design knowledge about finger and palm interacting positions. Through analysing the posture of hand holding cutlery, the cutlery handle could be separated into different concrete parts. The interact handle position was found through the video analysis and physical interaction with handle. The most interact areas with hands has been highlight (figure 4.10). In order to achieve the goal that help user grip easily and has an aesthetic appearance, the highlight areas adopt voronoi pattern and mesh surface. According to different hold postures on cutlery, the interact position will be separate by different areas.
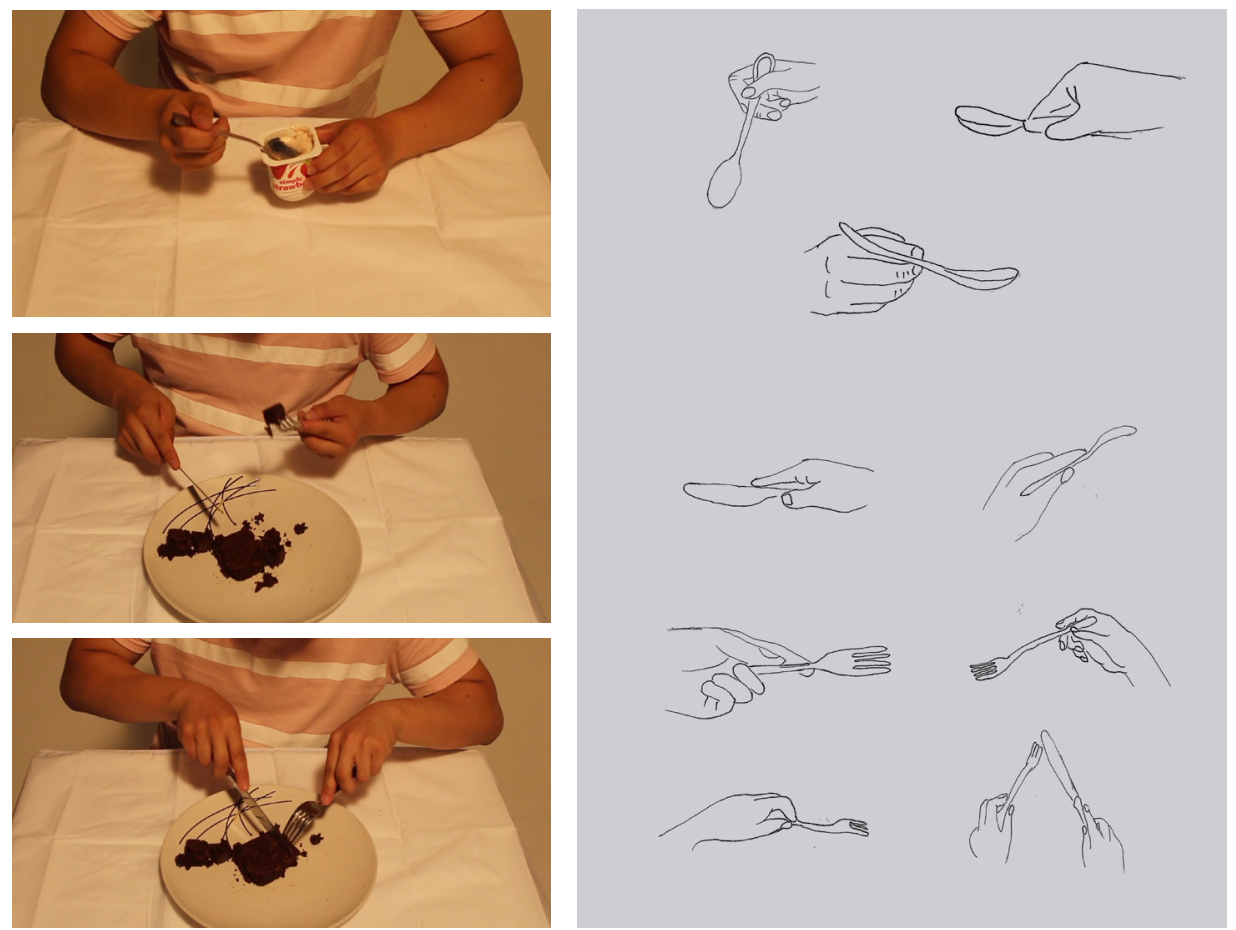

Figure 4.9 (left) Collecting hold posture through eating.

(right) Sketch of holding posture 

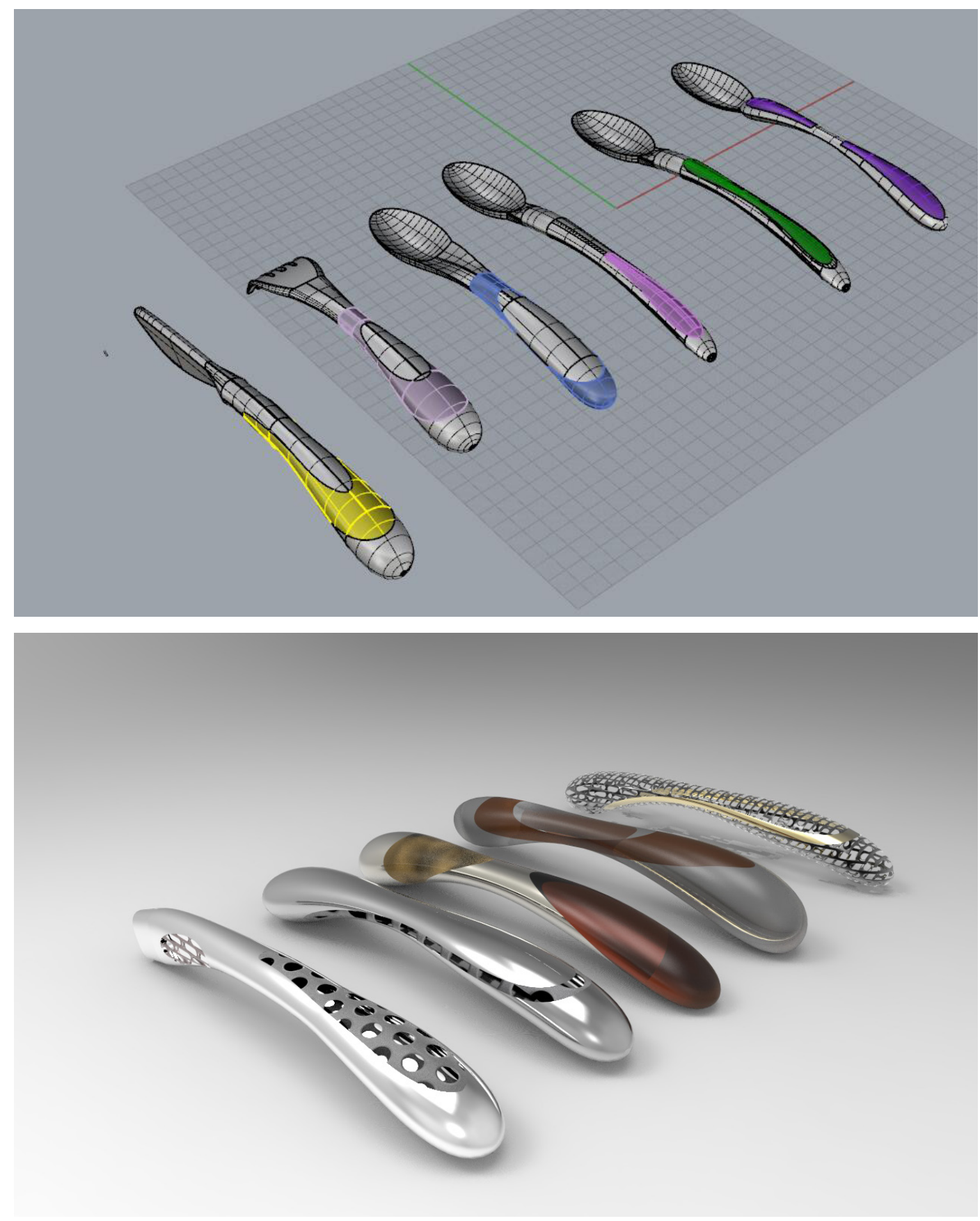

Figure 4.10 CAD highlight model (up) and digital rendering with voronoi patterns 

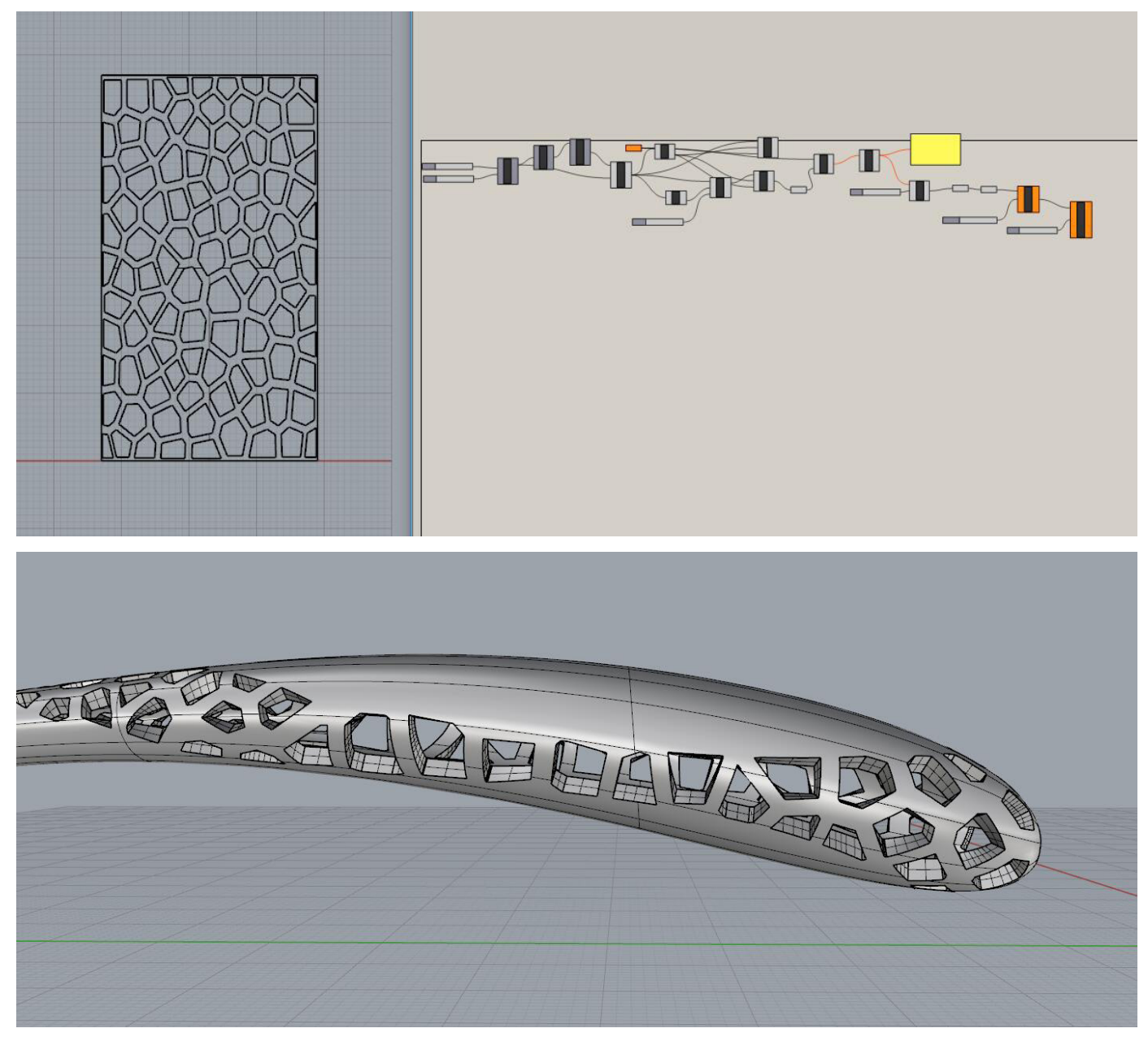

Figure 4.11 Using grasshoper to build the pattern and hollow out the handle. 


\subsection{Limitation}

During the prototyping process, several limitations restricted the design developments. The functional goal of this cutlery should be met when considering the balance of aesthetics and functionality in this design. However, the plastic material of 3D printing does not possess the feasibility and reliability for testing with real food (figure 4.10).

The ABS material printer also limited the printing quality. The mesh pattern of cutlery handle (figure 4.12) has been demonstrated that it will be difficult to print a real cutlery set and put them into physical eating activity.
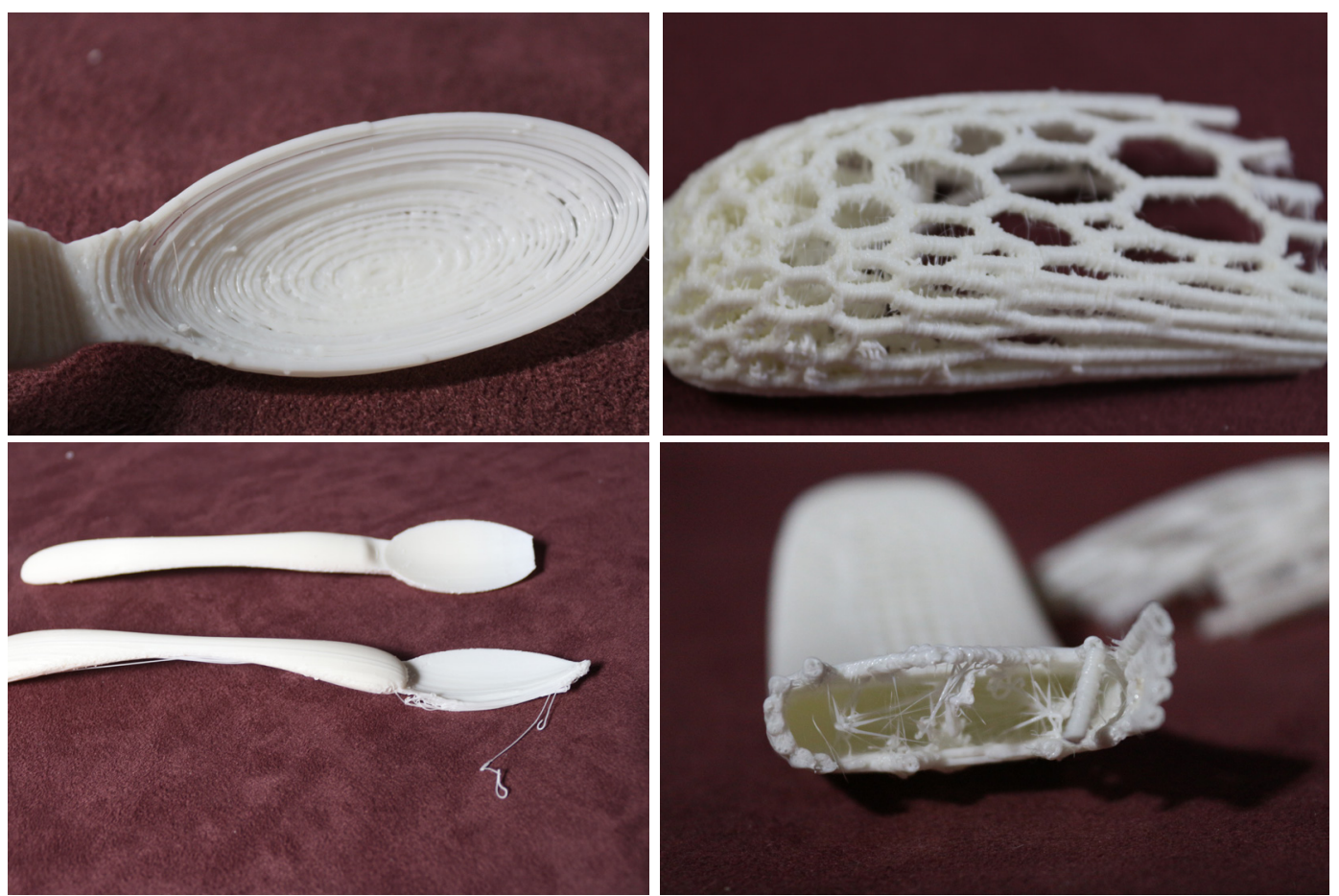

Figure 4.12 Failed 3D print

A promising way of testing the handle in eating task is to separate the handle with an existing front portion of cutlery (figure 4.13). However, this method will somehow conflict with the second aim of designing stigma-free cutlery, considering the combined cutlery appearance. 

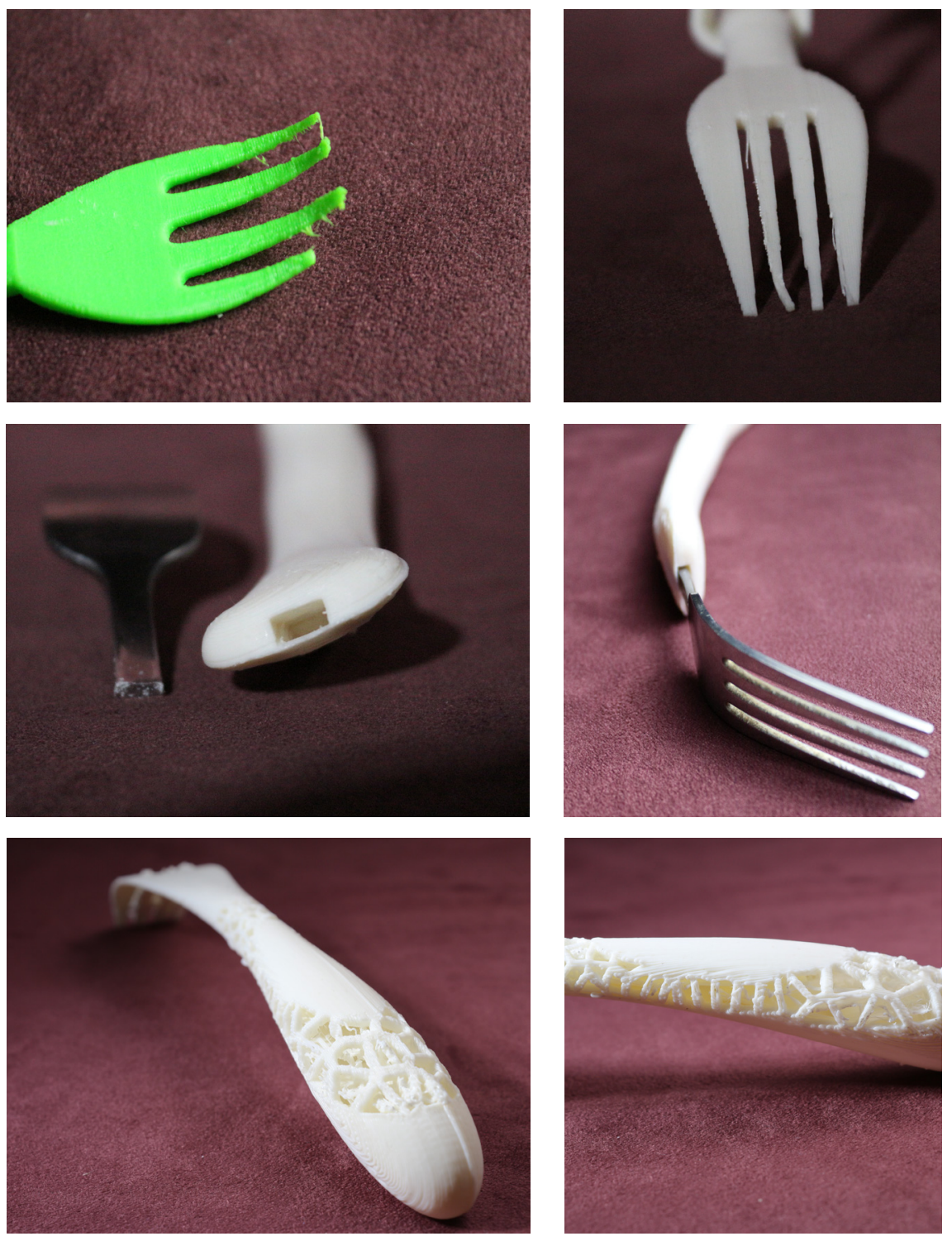

Figure 4.13 Failed front tine of fork (up), combined fork(middle), 3D print meshed fork (bottom) 

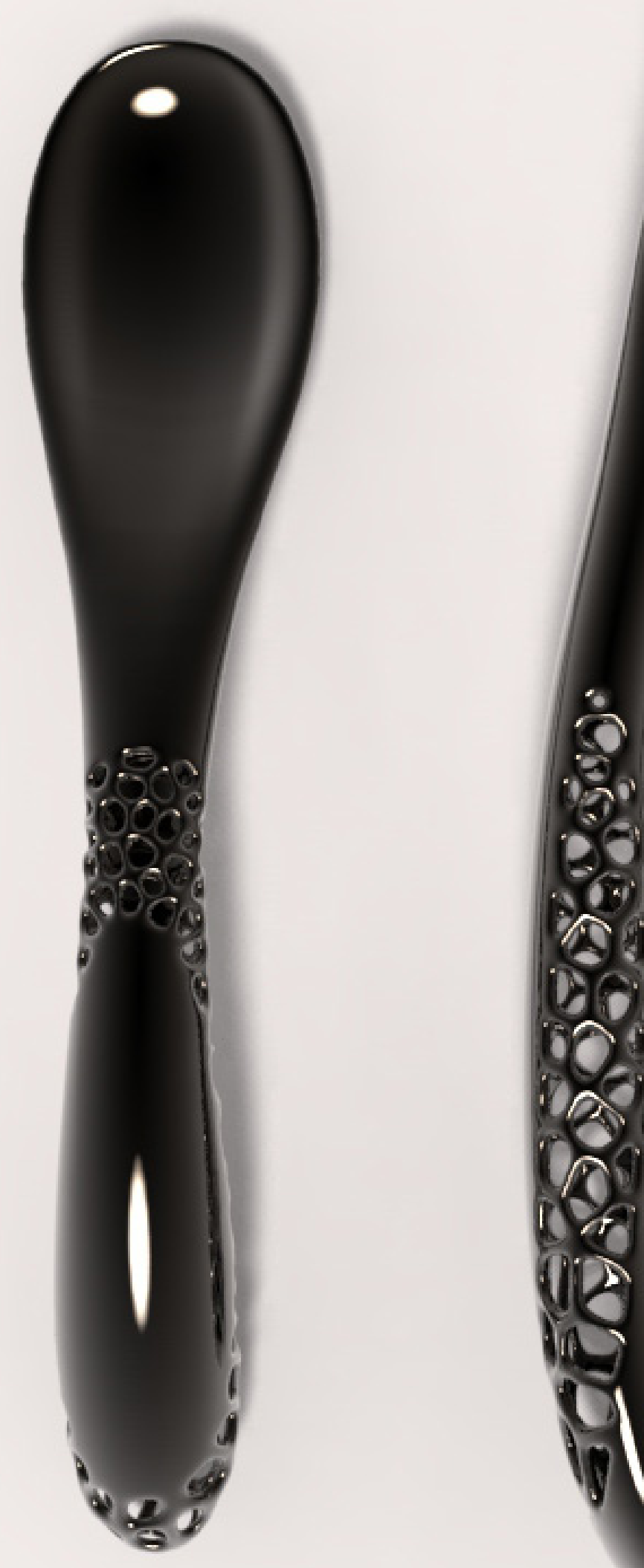

(N)
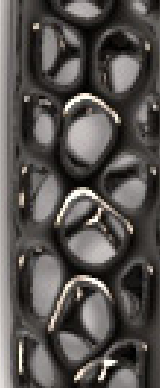

(Pios

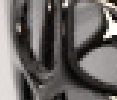

ins

0 


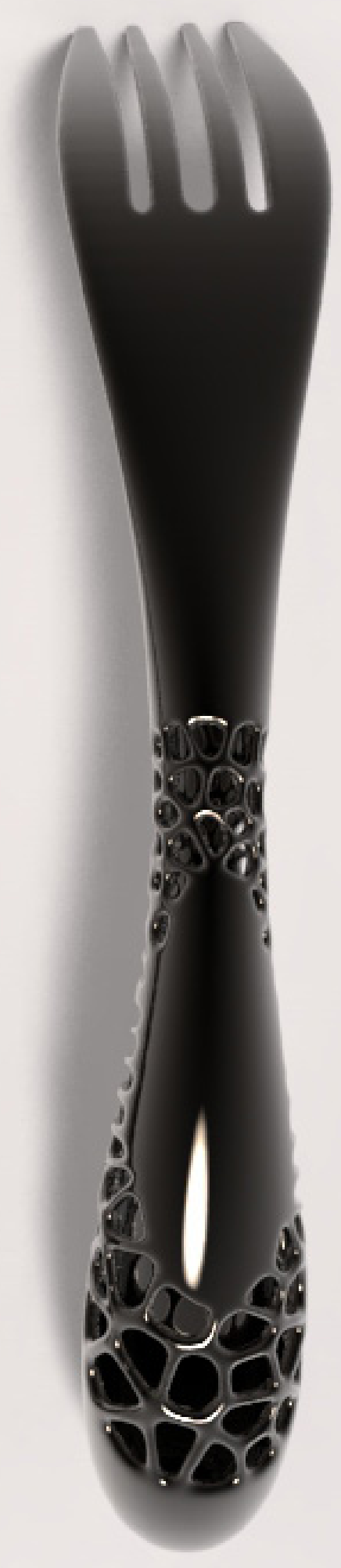




\subsection{Final design}

The final design comprised of a fork, a finger-wear, a spoon and a knife. The finger-wear was designed to focus on patients who have flexed index finger. The size of finger could customized with different size of index finger.

Due to the limitation of the printer and earlier 3D metal spoon, the final prototype is made by another metal printer. This printer adopts 'Selective Laser Melting' manufacturing process, which can produce a high-quality print. In the beginning, the prototype printed with a rough surface. Then there was a polish process to create a beautiful and high-quality print.

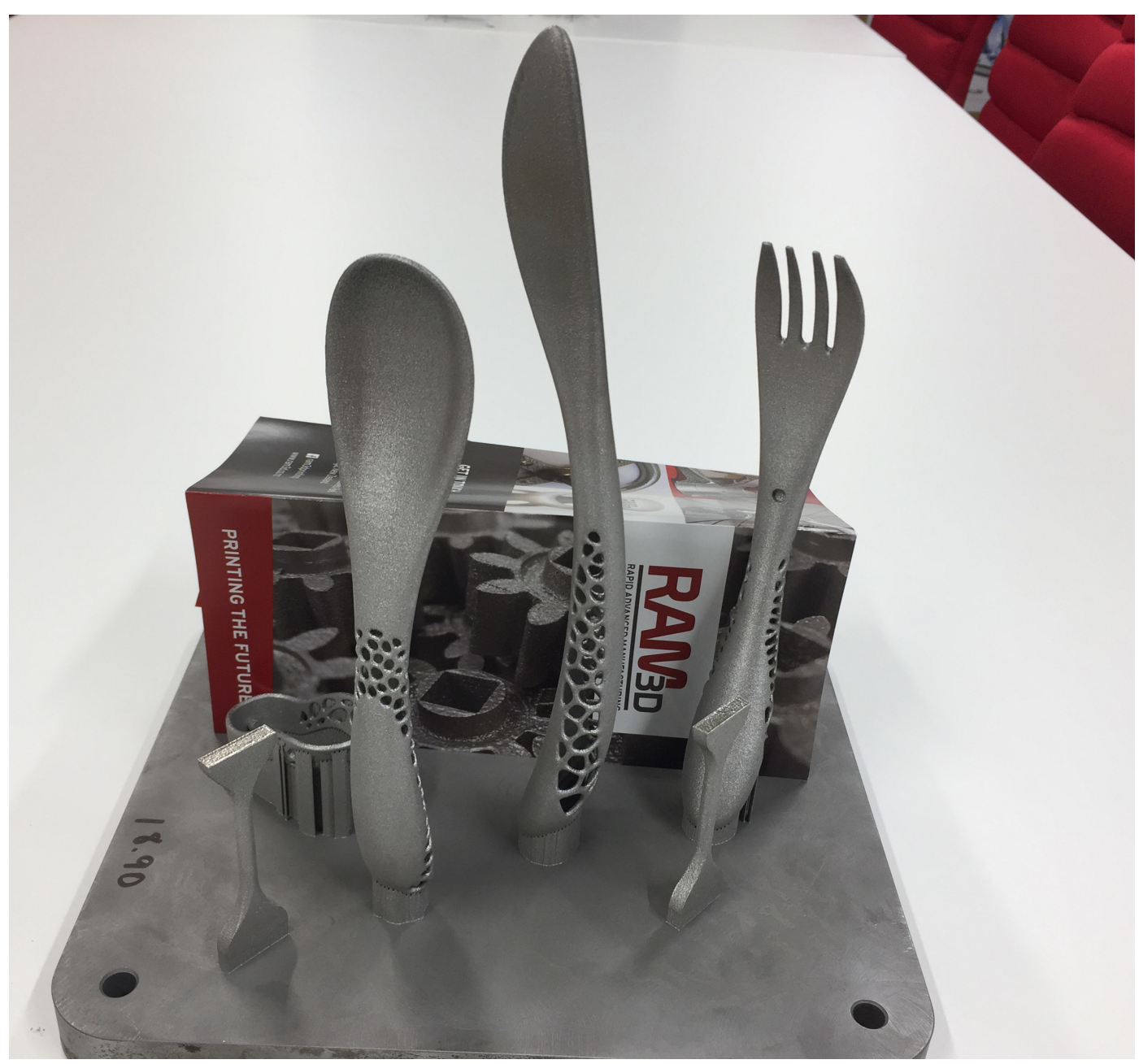




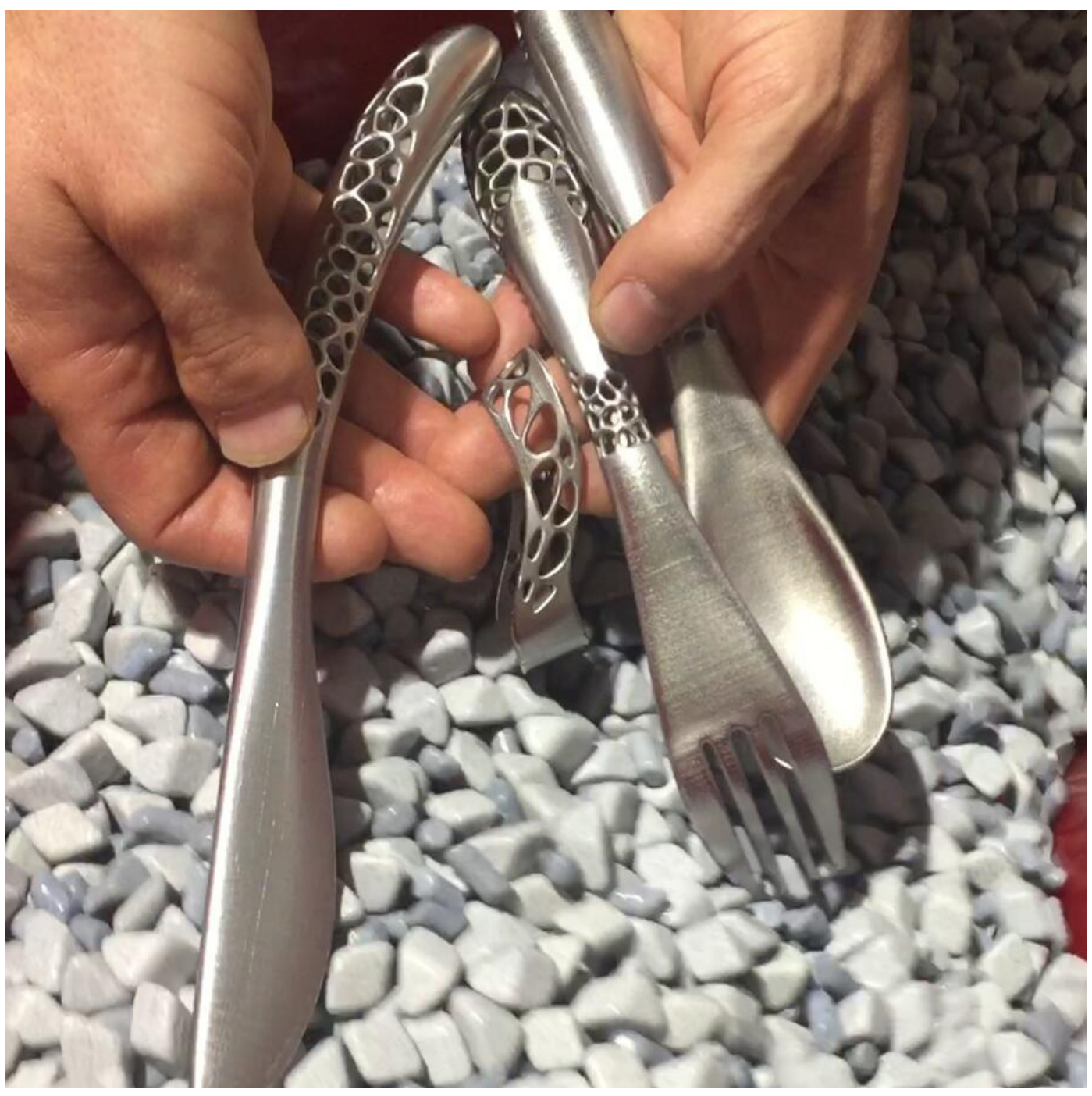

Figure 4.15 3D metal print and polished cutlery. The weight of each: 141g, knife

$118 \mathrm{~g}$, spoon

$100 \mathrm{~g}$, fork

$22 \mathrm{~g}$, finger-wear. 


\subsection{Angle of design}

The research findings indicate that shoulder weakness will restrict the lift movement and requires an accommodation for reduced range of motion and providing comfort for the user. For example, the different weaknesses of shoulders require a specific angle of spoon. The angled spoon can enable a user to reduce painful feelings when holding the cutlery towards to the mouth. The reduced painful feelings from affected shoulder will success the eating process. However, not all the patients have this problem. Therefore, a proposed scenario in this situation would allow users to customizable a suitable angled spoon. The proposed spoon design using 3D print technology intervention would allow for customizing a unique angle. A further investigation about angled spoon should conduct, and it should base on patients' health conditions.

The fork concept came from the historical cutlery analysis. Hundred years ago, the angle of fork front portion was straight. When the table manner rises up, people become critical about the cutlery functionality and aesthetic. The existing fork used in current life was developing from the straight fork. The curve of fork enable user has an aesthetic and effective motion to prick the food from plate. When designing a fork for stroke, the angle need to reshape and considered to be more effective. In order to allow users to use less strength to prick the food, the front of the fork neck was designed with a straight angle along with the handle. This angle makes the tine and the handle close to an angle of ninety degrees. This concept aimed to reduce user wrist pain. 


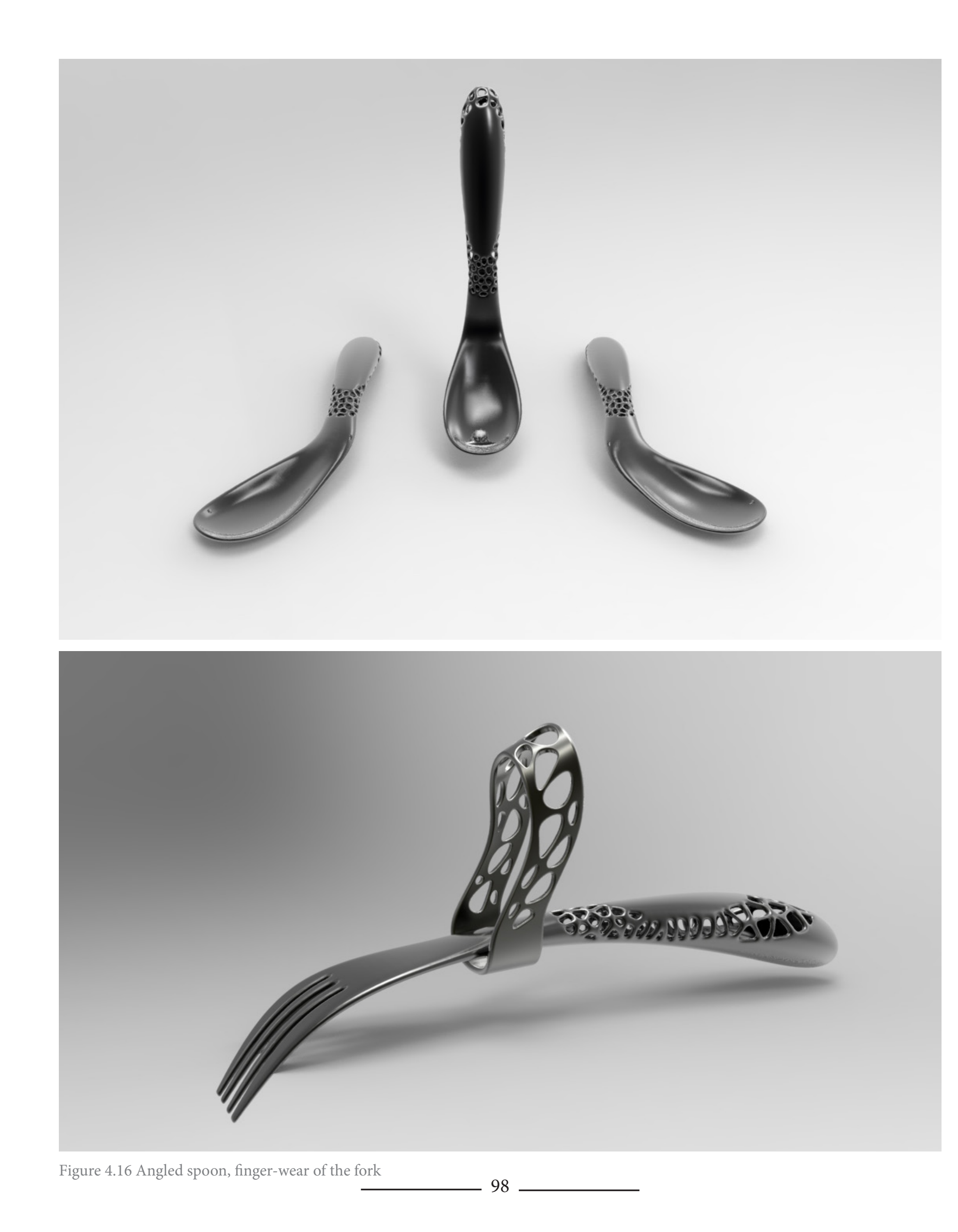



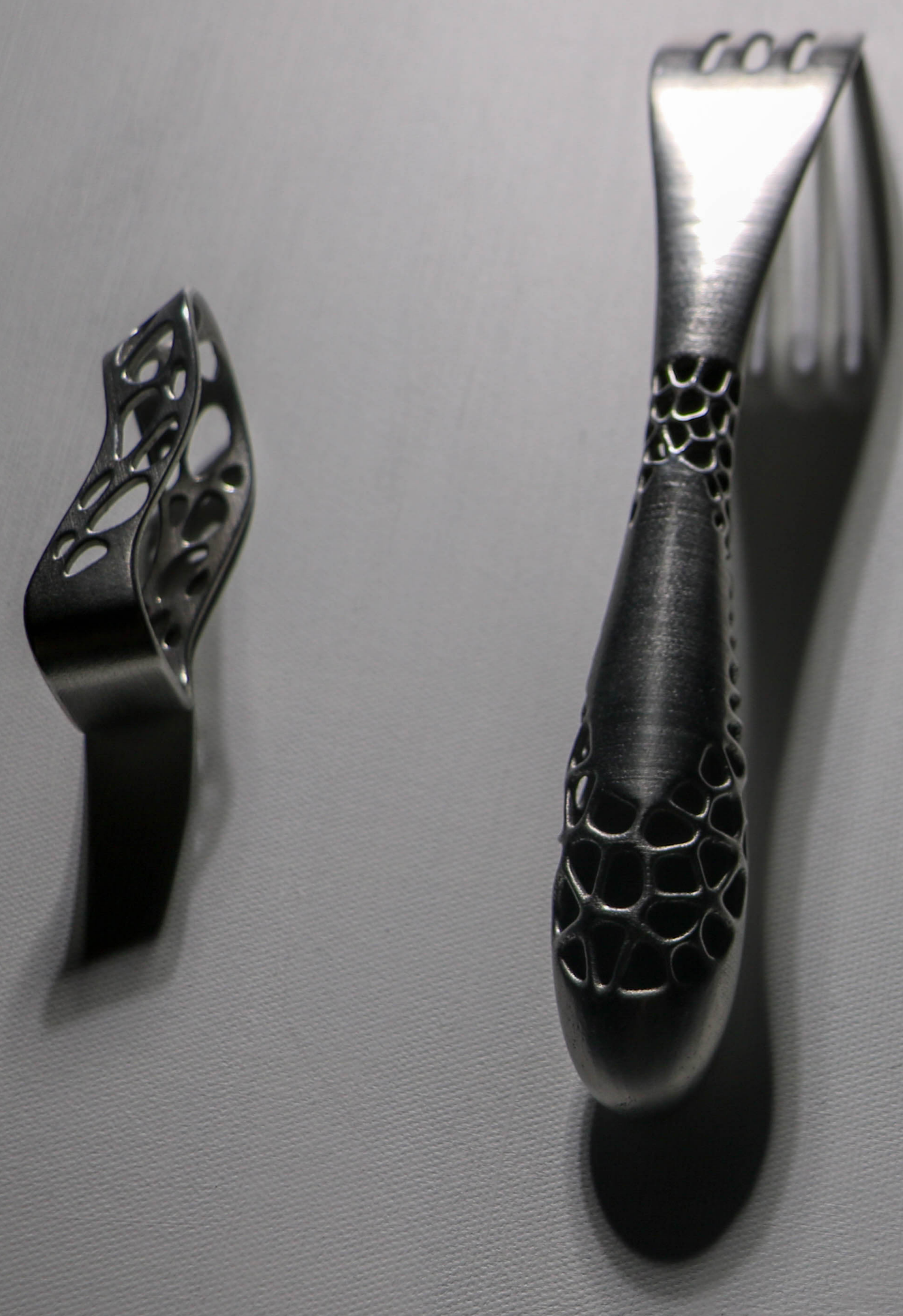


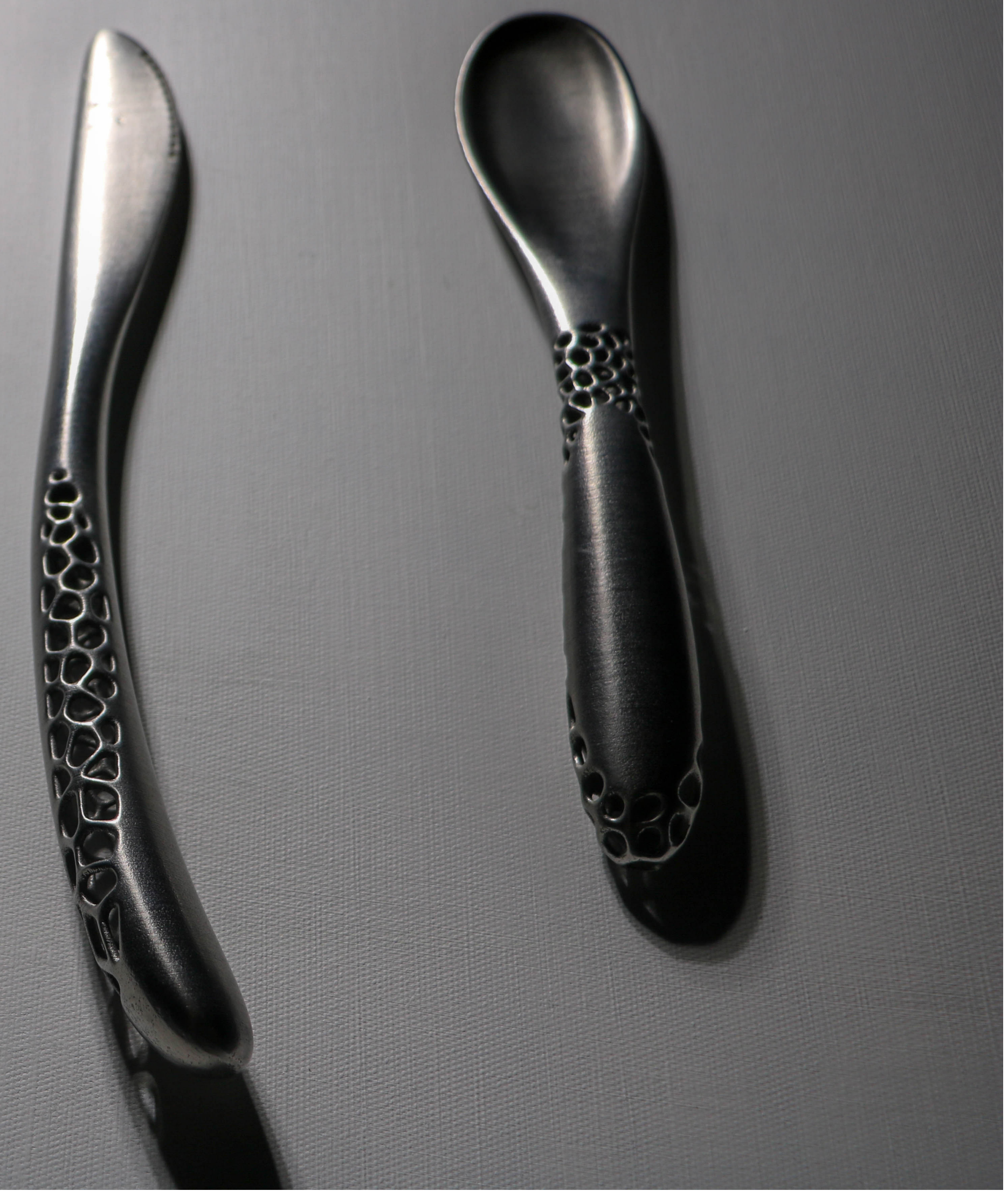




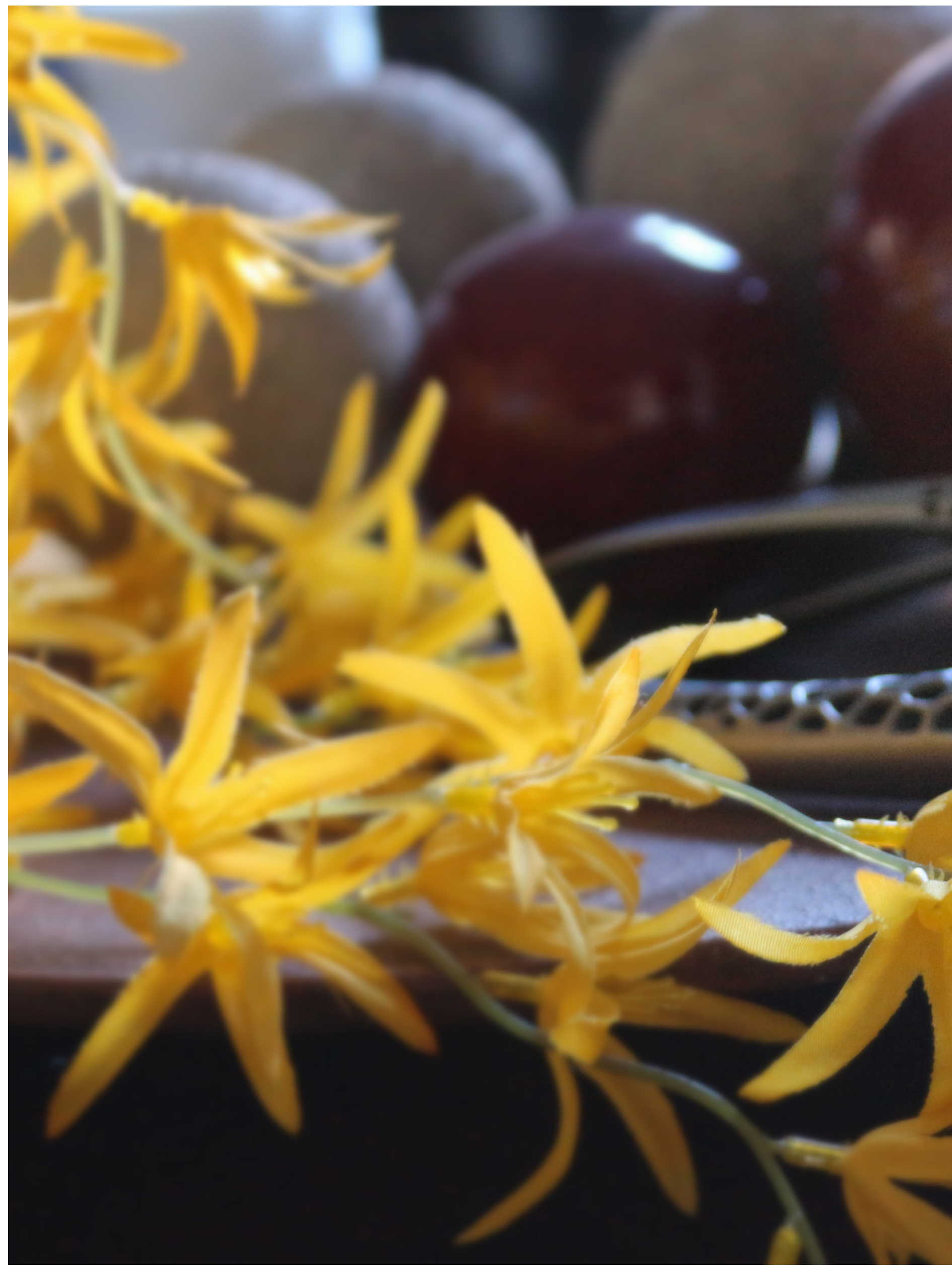




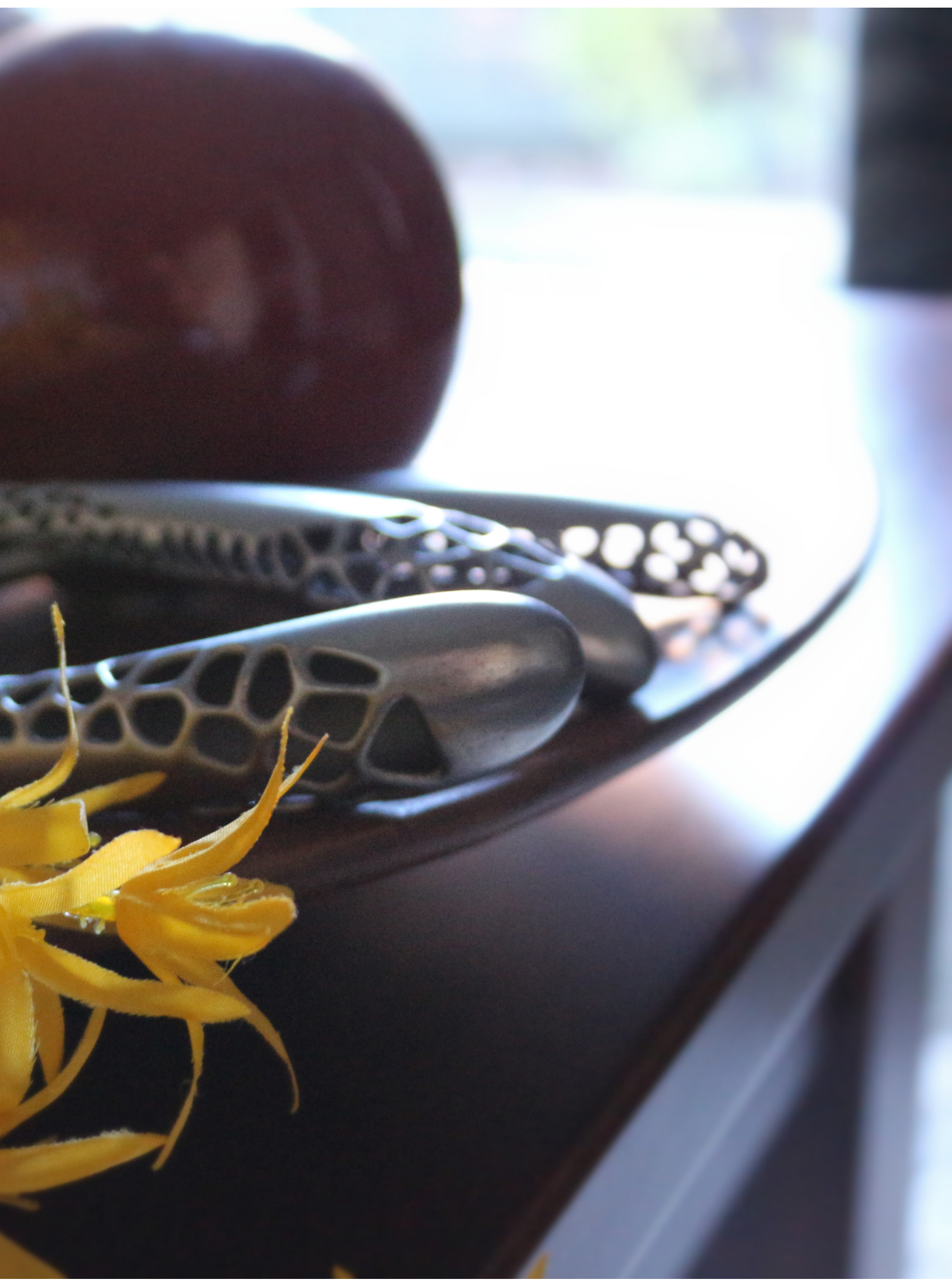




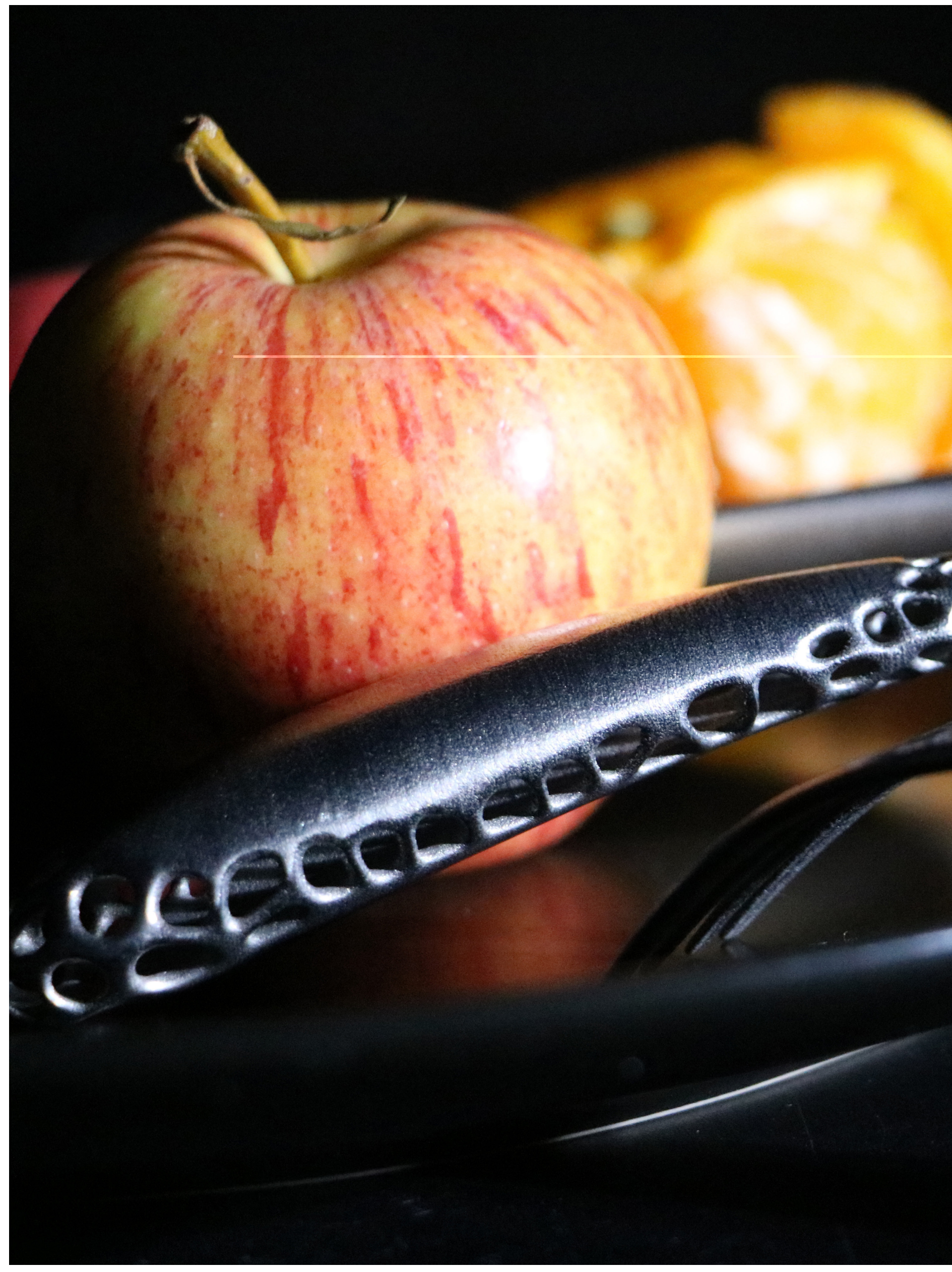




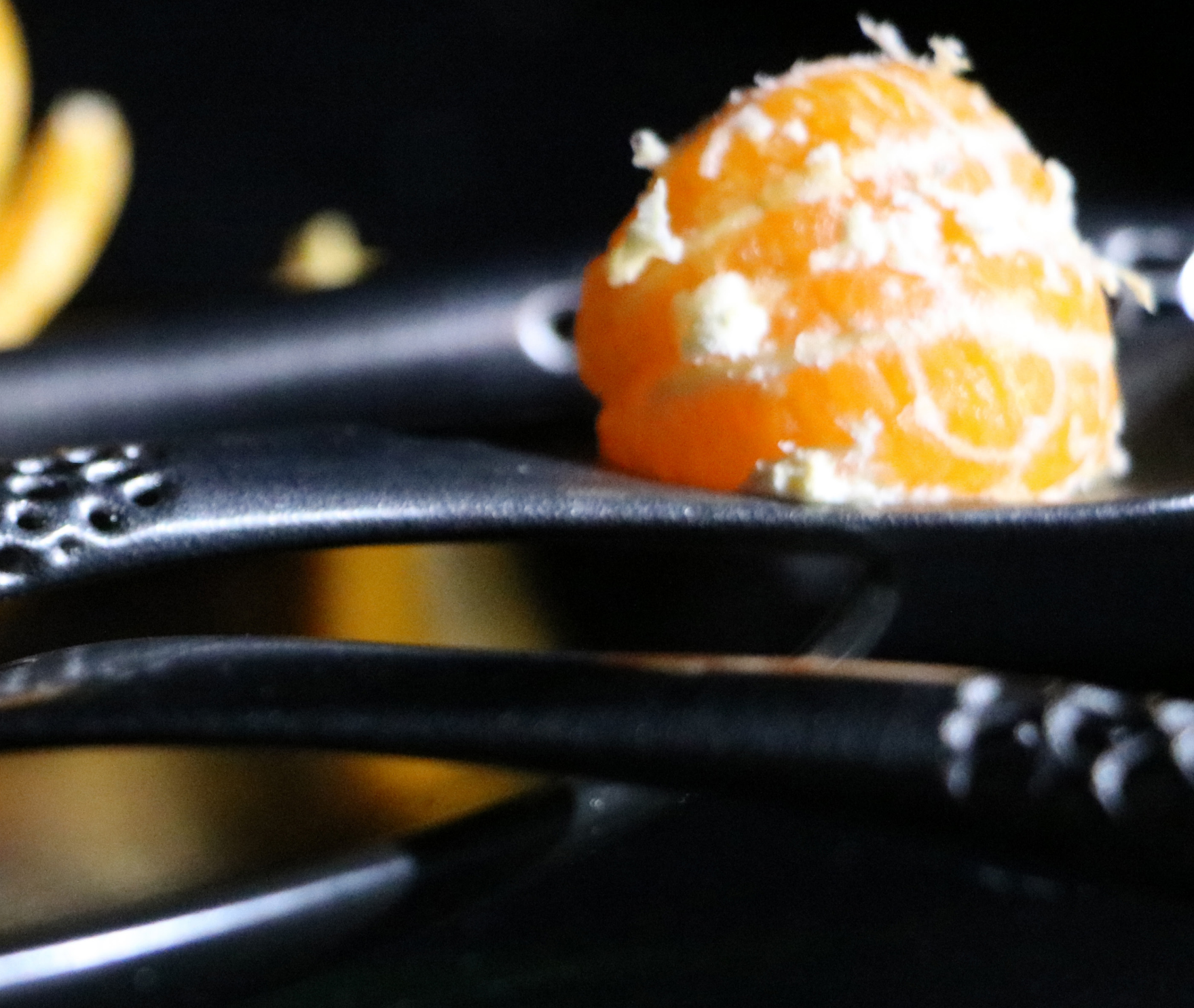





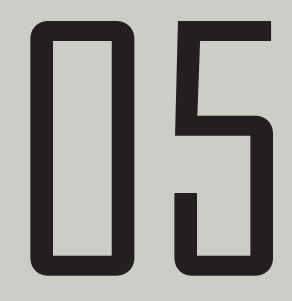

STIGMA TESTING AND ASSESSMENT 
Due to the limitation of stroke patient recruitment, testing on bystanders' perception and culture factors was conducted with the cutlery set. The perceived stigma (Vaes, 2014, p.53) from bystanders significantly incluences the user on their perceptions of product. Especially, the bystander will judge user's personality based on AT products.

This testing applied a revised appraisal questionnaire from 'the Product Appraisal Model for Stigma' (PAMS) (Vaes, 2014, p.147) and a revised 'the System Usability Scale' (SUS) (Brooke, 1984) questionnaire for addressing the stigma. The main aims of this testing intended to collect the bystanders' views and individual opinions on a cutlery set affected by different cultural factors. The participants were selected from different nationalities, in order to collect different opinions that were affected by cultural diversity.

\subsection{User profiles}

Participant A (male)

26 years old, An international student with Asian cultural background.

Participant B (male)

35 years ol, Asian cultural background, working in IT field and has been living in a Western culture country over 10 years.

Participant C (male)

A design student born and living in a Western culture country, has been educated in design study for many years and has a critical criteria about aesthetic design.

Participant D (male)

An international student with Asian cultural background, but the country he used to live in has a mix of world cultures. He also has critical criteria about aesthetic design.

Participant E (female)

A design student born and living in a Western culture country, she has been tutoring and working in the design faculty for a while. She has critical criteria about aesthetic design. 


\section{Product perception (sensing)}

1. The visual appearance of the cutlery set exhibits features that are discomforting for me.

Strong Disagree $\bigcirc \bigcirc \bigcirc \bigcirc \bigcirc \bigcirc \bigcirc \quad$ Strong Agree

2. The appearance of the cutlery set stops me from eating.

Strong Disagree $\bigcirc \bigcirc \bigcirc \bigcirc \bigcirc \bigcirc \bigcirc$ Strong Agree

3. User perceive an unacceptable expectation of cutlery set according to his quality standards.
Strong Disagree
$\bigcirc \bigcirc \bigcirc \circ$
O
$\bigcirc \bigcirc$
Strong Agree

4. The visual appearance of the cutlery set exhibits features that are discomforting for bystanders.
Strong Disagree
$\bigcirc \bigcirc$
O O
$\mathrm{O} \bigcirc \mathrm{OC}$
$\bigcirc \bigcirc$ Strong Agree

5. The visual appearance of cutlery set perception obstruct others in obtaining their goals.
Strong Disagree
$\bigcirc \bigcirc$

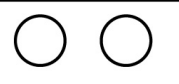
$\bigcirc$

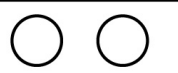
Strong Agree

6.The visual appearance of cutlery set conflict with bystander's expectations and beliefs following product use.
Strong Disagree
$\bigcirc \bigcirc \bigcirc$
$\bigcirc$
$\bigcirc$
$\bigcirc \bigcirc$ Strong Agree

7. The cutlery perception violate social or cultural taste.
Strong Disagree
$\bigcirc \bigcirc$
$\bigcirc \bigcirc$
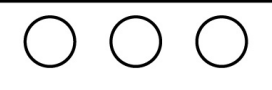
Strong Agree

8. The cutlery obstruct a culture in obtaining its goals.
Strong Disagree

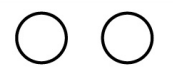
$\bigcirc$

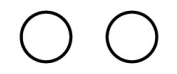
○
Strong Agree

9. The perceived cultural significance and value of the product or products aspects indicate change over time or in durability.
Strong Disagree
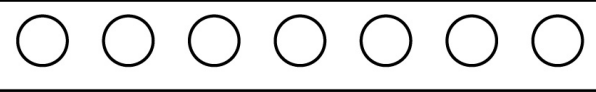
Strong Agree 


\section{Product in use}

1. The visual appearance of the cutlery set exhibits features that are discomforting for me when I using it.
Strong Disagree
$\bigcirc \bigcirc \bigcirc \bigcirc \bigcirc \bigcirc \bigcirc$
Strong Agree

2. The cutlery set does not help me to fit my eating purpose.
Strong Disagree
$\bigcirc \bigcirc \bigcirc \bigcirc$
$\bigcirc \bigcirc$
Strong Agree

3. There are dissonant experiences (imbalances between thinking/feeling and acting) when using this cutlery set.
Strong Disagree
$\bigcirc \bigcirc \bigcirc$
$\bigcirc$
$\bigcirc \bigcirc$
Strong Agree

4. Using the cutlery cause unease or a threat to others.
Strong Disagree
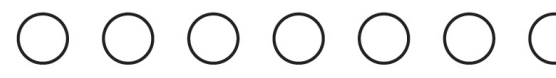
Strong Agree

5. Using the cutlery interfere negatively with the behavior of others; it prevent others from attaining their goals.
Strong Disagree

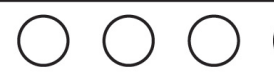
$\bigcirc$
$\bigcirc \bigcirc \bigcirc$
Strong Agree

6. Using the cutlery challenge the tolerance of bystanders.

Strong Disagree $\bigcirc \bigcirc \bigcirc \bigcirc \bigcirc \bigcirc \bigcirc$ Strong Agree

7. Using the cutlery conflict with cultural habits, rules or table manner.

Strong Disagree $\bigcirc \bigcirc \bigcirc \bigcirc \bigcirc \bigcirc \bigcirc$ Strong Agree

8. Using the cutlery indicate inappropriate cultural or societal behavior.
Strong Disagree
$\bigcirc \bigcirc$
$\mathrm{O}$
$\bigcirc \bigcirc \bigcirc$
Strong Agree

9. The cutlery still need to overcome thresholds in view of cultural or social acceptability.

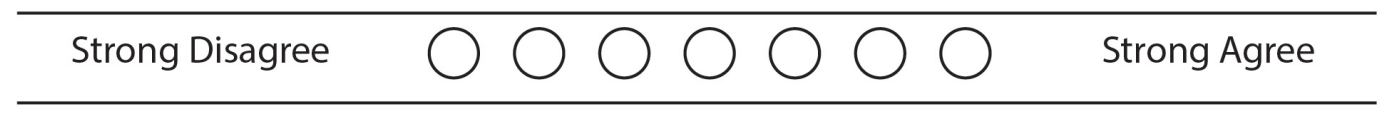

Figure 5.2 Revised questionnaire of "Acting". 


\section{Consequence of product use (Stigma)}

1. The appearance of the cutlery match with my personality and lifestyle.

Strong Disagree $\bigcirc \bigcirc \bigcirc \bigcirc \bigcirc \bigcirc \bigcirc \quad$ Strong Agree

2.The cutlery tolerated purely out of necessity or physical dependency.
Strong Disagree
$\bigcirc \bigcirc \bigcirc$
$\bigcirc$

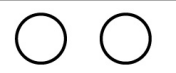
Strong Agree

3. The cutlery set appearance conflict with the user's expectations and beliefs following product use.

Strong Disagree $\bigcirc \bigcirc \bigcirc \bigcirc \bigcirc \bigcirc \bigcirc \quad$ Strong Agree

4. The visual appearance of the cutlery set match with attitudes of the bystanders.
Strong Disagree
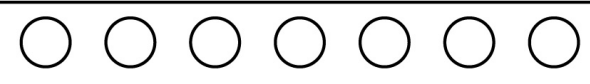
Strong Agree

5. The consequences of cutlery use harm the physical or psychological integrity of others.

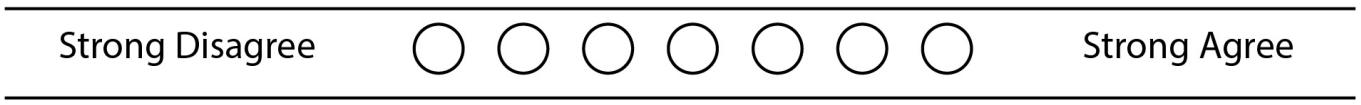

6. The cutlery set conflict with the bystander's expectations and beliefs following product use.
Strong Disagree
$\bigcirc \bigcirc$
$\bigcirc \bigcirc$
$\bigcirc \bigcirc \bigcirc$
Strong Agree

7. The visual appearance of the cutlery set and its user match with cultural preferences.

Strong Disagree $\bigcirc \bigcirc \bigcirc \bigcirc \bigcirc \bigcirc \bigcirc$ Strong Agree

8. The cutlery and its features comply with these goals and supporting regulations.

Strong Disagree $\bigcirc \bigcirc \bigcirc \bigcirc \bigcirc \bigcirc \bigcirc$ Strong Agree

9. The cutlery set conflict with the cultural values, expectations and beliefs following product use.

Strong Disagree $\bigcirc \bigcirc \bigcirc \bigcirc \bigcirc \bigcirc \bigcirc$ Strong Agree 


\section{The System Usability Scale \\ (SUS)}

1. I think that I would like to use this product frequently.

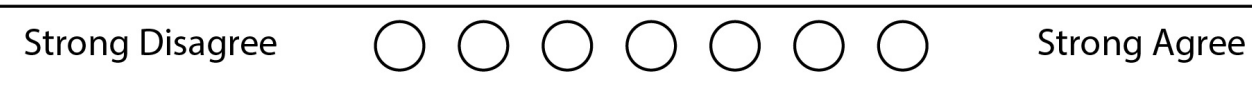

2. The cutlery set does not help me to fit my eating purpose.

Strong Disagree $\bigcirc \bigcirc \bigcirc \bigcirc \bigcirc \bigcirc \bigcirc \quad$ Strong Agree

3. I thought the cutlery set was easy to use.

Strong Disagree $\bigcirc \bigcirc \bigcirc \bigcirc \bigcirc \bigcirc \bigcirc \quad$ Strong Agree

4.I think that I would need help from a technical person to be able to use this cutlery set.
Strong Disagree
$\bigcirc 0$
$\bigcirc \bigcirc$

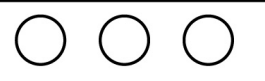
Strong Agree

5. I found the various parts of the cutlery set were well integrated.
Strong Disagree
$\bigcirc \bigcirc$

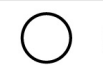
$\bigcirc \bigcirc \bigcirc$
$\bigcirc$ Strong Agree

6. I thought there was too much inconsistency in this set of cutlery.
Strong Disagree
$\bigcirc \bigcirc$
O
$\bigcirc \bigcirc \bigcirc \bigcirc$
Strong Agree

7. I would image that most people would learn to use this cutlery set very quickly.
Strong Disagree
$\bigcirc \bigcirc \bigcirc$

9. I felt very confident using the cutlery.
Strong Disagree
$\bigcirc \bigcirc$

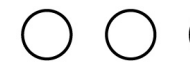
$\bigcirc \bigcirc \bigcirc$
Strong Agree

9. I felt very comfortable using the cutlery.
Strong Disagree

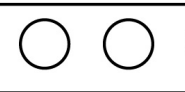
$\bigcirc \bigcirc \mathrm{O}$
$\bigcirc \bigcirc \bigcirc$
Strong Agree

10. I needed to learn a lot of things before I could get going with this set of cutlery.
Strong Disagree

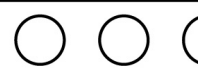
$\bigcirc$
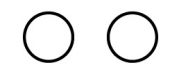
$\bigcirc O$
Strong Agree 


\subsection{Stigma test}

To explore how the cutlery affect people's perception of eating tools, the testing investigated users' visual perception, tactile perception and their stigma response to the cutlery set. The testing mixed the questionnaire and semi-structured interview (Wood, 1997) to let the participants verbalize their thoughts and opinions regarding their interactions and subjective experiences with the prototypes. The testing was supplemented with self-reporting, which took the form of questionnaire (figure 5.1, $5.2 \& 5.3$ ) that included a scale for subjectively assessing perception of stigma. The process after the reading of information and the consent forms signing was as follows:

1. The participant sat in front of the table. The researcher brought the cutlery set to the participant.

2. The participant was asked to look at the design from a distance. During the observing stage, they were asked to fill out the sensing questionnaire (figure 5.1).

3. The second stage is that participants hold the cutlery in their hand and were asked to imagine they were using the cutlery while eating.

4. After finishing the second questionnaire, the first evaluation questionnaire is given to the participants for collecting the initial feedback from the user. (figure 8.3)

5. The participant was asked to fill out the questionnaire (figure 5.1, 5.2, 5.3 \& 5.4) . In the questionnaire progress, they were allowed to pick up and inspect each of them. In the end stage participants were asked to fill out the SUS questionnaire and were told the questionnaire would be used to review through the whole process of the testing.

6. After the questionnaire investigation finished, the prototype was taken back to the cutlery case.

7. Through the beginning to the end, there the participants had the opportunity to ask questions about the questionnaire if they want to know more information about the design and appraisal model questions. 


\subsection{Analysis \& Review the testing process}

The review of the stigma testing aimed to reveal the insufficient aspects of the cutlery during user testing and data collecting. The testing was conducted in two environments:

1. Domestic environment (Participant A \& B).

2. Public environment (Participant C, D \& E).

The testing results are presented in two parts:

1. The results of the appraisal questionnaire.

2. The question that participants asked researcher during the process.

Before starting the testing process, participants A, B, C and D previously knew this study through the information sheet. However, the fact that the target user of this research primarily was comprised of stroke patients had influenced participants before they filled out the questionnaire. Then, they raised a few questions about differences between them and the target group. For example, they thought there were some questions that were not for them although they had no problem answering them. To people who have not had a stroke, the finger-wear would not be used in eating. Thus, the finger-wear did not appear in the whole testing process. 

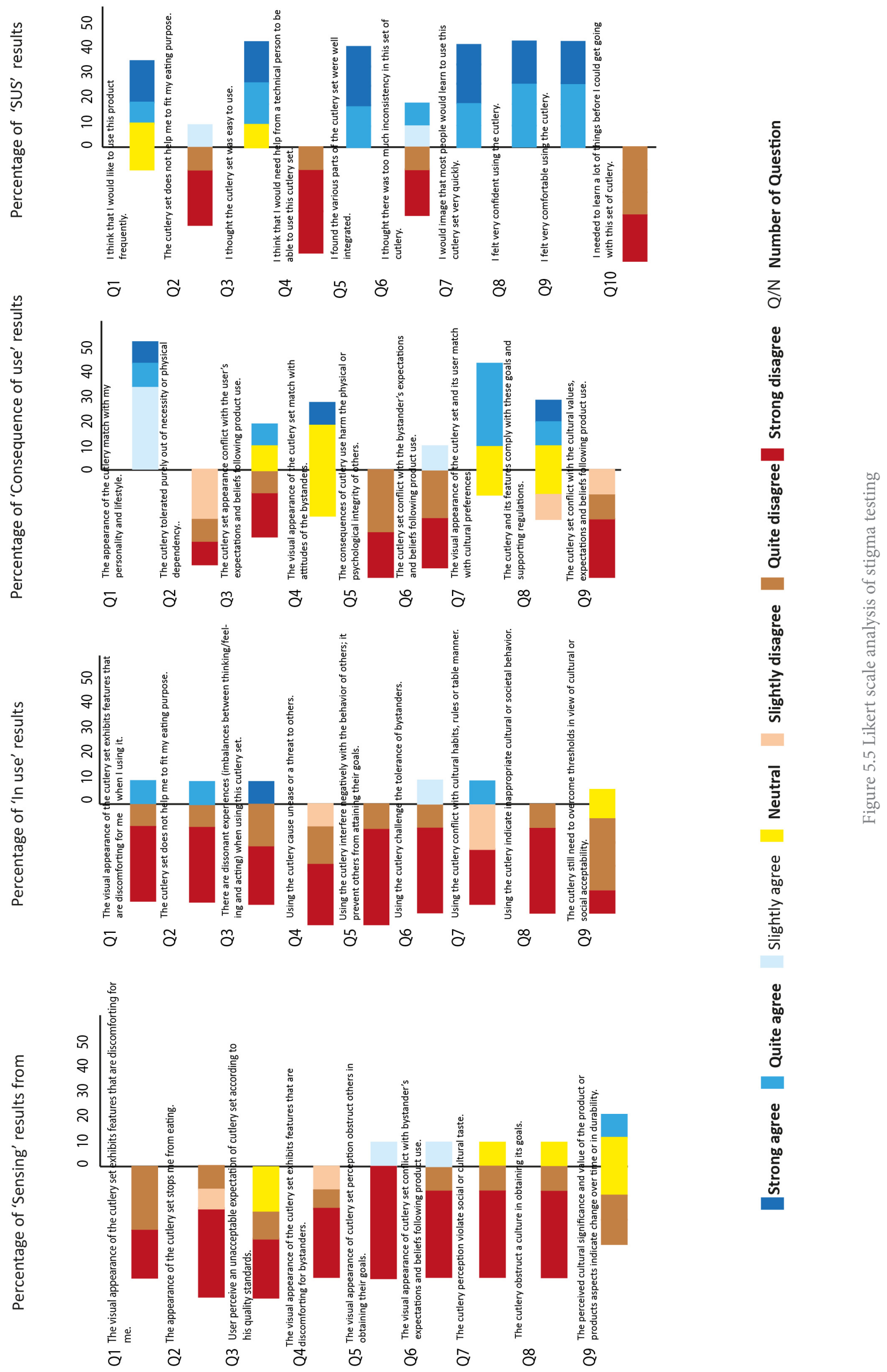
In order to gather objective feedback, the analysis began with a summary of the participants' questionnaire. Most of the answers in the questionnaire were found to be positive and objective. For instance, most of the participants choose 'quite agree' or 'strongly agree' in the question 'I thought the cutlery set was easy to use'. Similar to others' questions, most answers demonstrated that the cutlery set received expected positive feedback and reached the goal of designing a stigma-free cutlery set.

However, there were several answers in the questionnaire that demonstrated that participants had an uncertain attitude and neutral opinions. The summary is as follows: 


\section{Participant A}

Neutral opinion in Question 9 (Q9) of 'Sensing' section.

\section{Participant B}

Neutral opinions in Q1 of SUS section, and in Q4, Q7, Q8 of 'Consequence of product use section'.

\section{Participant C}

Neutral opinions in Q3 and Q9 of 'Sensing' section. Neutral opinions in Q4 and Q8 of 'Consequence of product use' section.

\section{Participant D}

Neutral opinion in Q4 of 'Consequence of product use'.

\section{Participant E}

1. SUS section.

Neutral opinion in Q1and Q3. Slightly disagree opinion in Q6.

2. Consequence of product use.

Neutral opinion in Q4 and Q7. Slightly disagree opinion in Q2, Q8 and Q9.

3. Product in use section.

Neutral opinion in Q9. Slightly disagree opinion in Q4 and Q7.

4. Sensing section.

Neutral opinion in Q3, Q7 and Q8. Slightly disagree opinion in Q2 and Q4. Slightly agree in Q5. 


\section{Results review}

The analysis started from collecting the information from each answer and analyzing uncertain answers. There was no extremely negative and poor feedback in the testing results while collecting each of the questionnaires. In the sensing section, participants chose 'disagree' to admit the cutlery set brings a stigma emotion to them. Participants were surprised about the cutlery appearance when they first saw it. Although participants with an Asian cultural background were more interested in the appearance and presented that they would like to have such a cutlery set for eating, this might relate to their experiences using cutlery. Most Asians usually use chopsticks in eating, and a spoon would be used in scooping soup or liquid food. This cutlery set includes a knife and fork and it does not provide a pair of chopsticks. Comparing with the frequent use of a Western cutlery set, most Asians would like to choose chopsticks for eating. Their attitudes to the Western cutlery are that they are more likely to own them rather than use them. Most Asian still wish to use chopsticks if they are not eat with a westerner. In the conversation with a participant who came from Asia, they said they would treat this cutlery set as a decoration in the kitchen if they do not use them frequently.

Participants from New Zealand culture were more focused on functionality and bystander aspects. Especially Participant E elicited a lots of deficit aspects through answering the question. A lot of neutral opinions and uncertain attitudes in this testing process demonstrated the revised questions did not fully specific that enable to answer with a certain and clear attitude. Several 'bystander' questions in this testing showed that the bystander's views need to be tested in an actual eating environment. It is difficult to measure the bystander's emotion without them observing a real user using the cutlery. 
Participants from New Zealand culture were more focused on functionality and bystander aspects. Especially Participant E elicited a lots of deficit aspects through answering the question. A lot of neutral opinions and uncertain attitudes in this testing process demonstrated the revised questions did not fully specific that enable to answer with a certain and clear attitude. Several 'bystander' questions in this testing showed that the bystander's views need to be tested in an actual environment. It is difficult to measure the bystander's emotion without them observing a real user using the cutlery.

To conclude this, the review summarized that people's different cultural backgrounds will influence their criteria on cutlery appearance. The long-term use cutlery experiences and domestic table manners primarily formed preconceived notions on their perspectives about whether it is good cutlery. On the other hand, there is no certain answer for all participants that expressed the participant will use them and without abandoned case in long-term usage. When reviewing the testing process and the results, participant $E$ in this testing especially presented a different perspective in receiving this set of cutlery from comparing with the previous male view. An assumption here is that the gender role and personality will also influence participants' preference on cutlery sets. 


\section{Dishwasher test}

There was another testing process about the usability and performance in a domestic environment. In previous Chapter 1 and clinicians' feedback collection, the rubber and sticky material gave a poor performance when being used as repeat utensils. Thus, the usability about repeat use and material effectiveness in real dining use testing was conduct. Firstly, the testing involved the cutlery set in a washing test in a dishwasher. Secondly, the cutlery set was used for interacting with food.
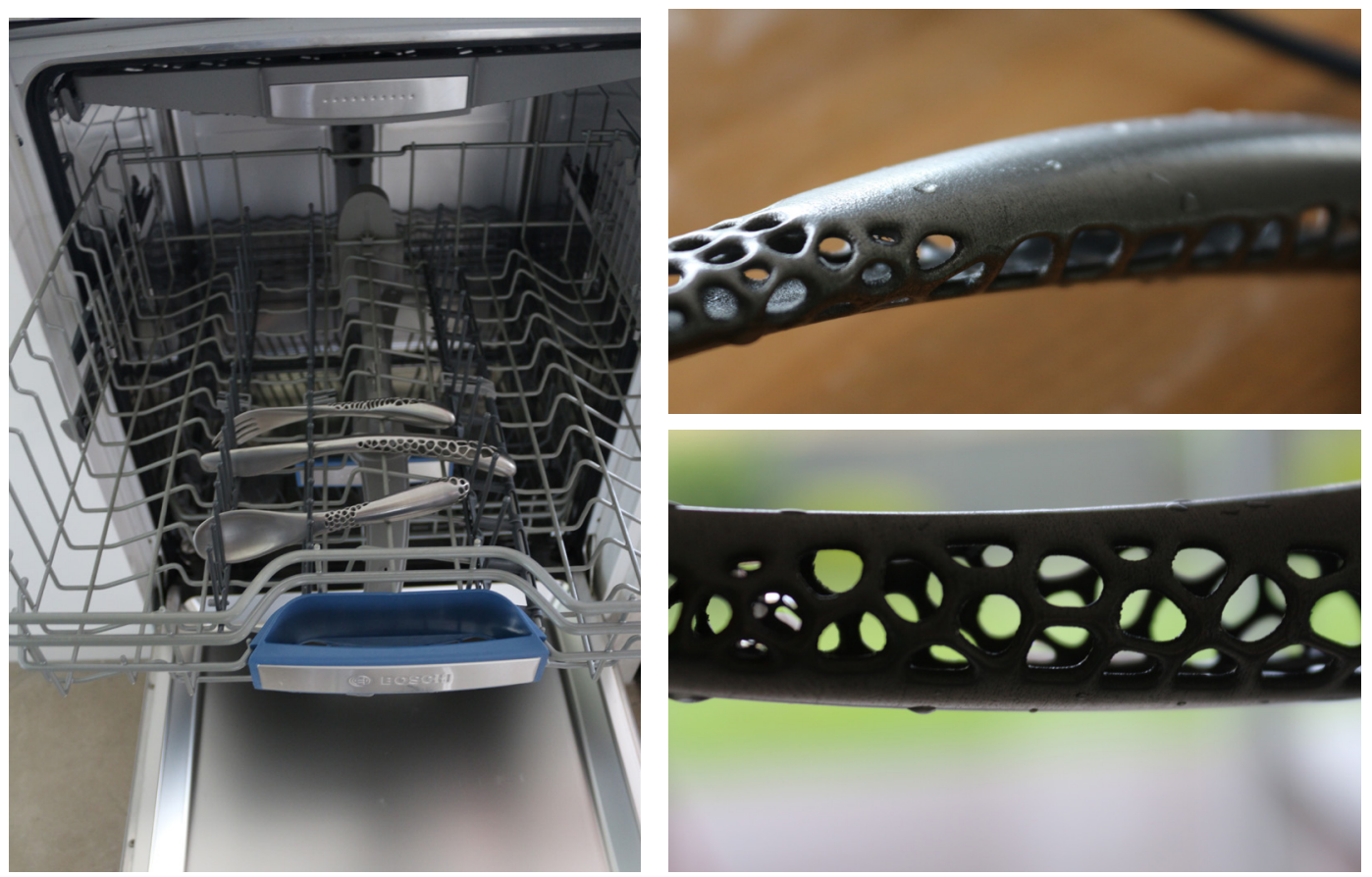

Figure 5.6 Dishwash test 


\section{Limitations}

There were several limitations and issues with the stigma user testing that became apparent during the testing sessions:

1. The stigma testing was inherently qualitative and limited by a sample size of three. Intended testing content was comprised of other AT cutlery, which was in order to generate inherent bias interest to the project.

2. The inherent bias from the researcher gave the questionnaire a vested interest in seeing the participants be positively surprised and have a pleased emotional experience.

3. The testing process was conducted with participants individually, so the subjective interpretation of each might affect their mind when they started to fill out the questionnaire. An open discussion and critiques might provide more objective results. 



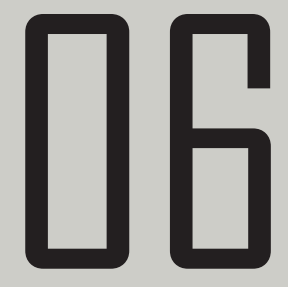

DISCUSSIOUN 
In addressing the question "How can the design of a set of cutlery minimise stigma and address functional requirements of stroke patients", this research focused on two key considerations: how to address stigma in the design of cutlery and how the designs can functionally help stroke patients eat. The cutlery set is developed as assistive technology (AT) cutlery, specifically to facilitate stroke patients when eating. In addition to that, the aim of the research was to provide a significant contribution in addressing stigma in an AT cutlery set. The minimized stigma goal requires the cutlery to be acceptable for people who have not had a stroke. A minimized stimg cutlery set should attract user and please users whether they have stroke or not.

The background research adopts several approaches that are different from Torren's study (2013). In order to address product-related stigma among users, this research emphasizes Vaes' (2014) stigma study, which has significantly contributed an appraisal frame that helps to design a stigma-free cutlery. Through the stigma appraisal criteria, this research has formed several design criteria that strongly support the design frame and future works. Similarly, Renda (2015) proved that gender and personalities also influence the perception of users to accept AT cutlery. Renda's study specifically explored users' psychological emotions and the personal perception behind experimental AT cutlery experiments.

This research selected several approaches in order to design a stigma-free cutlery set. Vaes' (2014) stigma theory provided a strong basis for this research through the Product Intervention for Stigma. It has been considered and revised in this research. Through this research background and clinicians' feedback, a lot of intervention and design approaches must be combined with the actual situations and physical facts. In the design process, an important fact is that the aesthetic of AT products for people who have disability cannot be fully equal to the aesthetic of mainstream products. Functionality is considered throughout to be and will be a primary aim in the whole design process. 
The user, the bystander and the culture were the main starting points when designing this set of cutlery. Undoubtedly, it will go back to the appraisal factors when testing the stigma theory. The stigma testing proved that the participants' cultural background and their different eating habits significantly influence their perceptions about a new set of cutlery. Especially, the stigma test was conducted by one-to-one interview, but a group discussion would also be helpful for the test.

\section{Evaluation through design criteria}

\section{Summary criteria}

- The design should allow user to use in a domestic environment and public (mainly in restaurant) environment.

- The design should provide an aesthetic appearance to all users.

- The design should be a stigma-reducing AT cutlery.

- The appearance of design should similar to a conventional cutlery shape that would not make user feel strange considering their eating experience.

- The design should be easy to setup and use, reducing the complex introduction of how to use.

- The interaction of this design should make user feel it is effective in usage.

- The finger-wear should help patients who have flexed index finger.

- The finger-wear should has a similar visual language to the cutlery set.

- The design should enable user to keep and use in a long-term.

- The cutlery set must adapt to patients at different ability levels. This would help patients to continuously use the product.

- The design should adapt to different stages of stroke.

- The aesthetic of the design should reflect its usability through the interaction.

- The design should look like an elegant cutlery product.

- The design should encourage the user to use it confidently.

- The design should elicit pride and avoid embarrassment. 
Through reviewing the background research and design research, this study has informed several criteria that could examine the design. This design has provided a new version of AT cutlery. Although this cutlery set did not take a patients user testing in order to address visible stigma and functionality, it brings a new perspective to those medical products associated with product-related stigma. In the design process, this design selected the 3D print technology to be the main intervention. However, it has demonstrated that several physical aspects of this design and the technology intervention can not fully fill. Examples include the weight of each prototype, the lock of the finger-wear, and the edge of the knife.

The lock of the finger-wear was proposed to lock together with fork successfully. But the detail of the lock did not reach the goal of accuracy. It made the lock can not attach to this fork in a stabilized status. Further subsequent traditional assistance may help the design to be impeccable. Particularly, the knife edge could be sharpened by a whetstone or diamond stone. Also, there was an uncertain design purpose of knife design in the early stage. In daily life, there are various kinds of the knife in different eating activities. For example, a dinner knife is different from a dessert knife. There was a proposed investigation of user eating tools preferences and eating habits should be conducted. However, due to the ethics limitation, the research was not able to close to their lives.

\section{Functional requirements}

The main objective of this project is to develop a cutlery set and help with user eating. The reshaped streamlined handle enables users to accomplish eating tasks. Initial target users are comprised of stroke patients and people who have not had a stroke. Due to the time limitation, user testing was only conducted to test with people who have not had a stroke. The 3D metal print in this research has been found acceptable and allows use in domestic environment. 
The dishwasher test proved this cutlery set to be safe to use in a continuously employed purpose. The integral body structure in this functional requirement has received positive feedback during the testing. There were several requirements that the knife prototype and fork did not in fact meet. Firstly, the knife edge was found to be not sharp enough. This reflects that traditional manufacturing skill is still important and necessary to effective cutlery. It is possible to make a development work by traditional manufacturing methods. Secondly, the knife handle was designed to be too long. It influenced the visual effect and was a discomforting feature compared with the whole set.

\section{Aesthetic requirements}

Another main objective of this project is to create sigma-free cutlery when it is included in the AT product scope. The design has removed a few medical semantics when considering the feedback from literature and clinicians' feedback collection. An integrated stainless steel body structure design demonstrated that the aesthetic expectation is for stigma-free cutlery and a modern design trend. But the 3D printed stainless steel material applied in this set of cutlery has found that it causes an overweight than a regular set of cutlery. Comparing this set cutlery with others AT cutlery sets, this set of cutlery is still weightier than them. From the researcher view, the weight of whole cutlery set influences the aesthetic aspects.

In order to achieve the goal of evaluation, a revised questionnaire from the Product Appraisal Model for Stigma (PAMS) model and the System Usability Scale (SUS) was involved in the usability during the stigma testing. The design elicited a positive emotion and fewer stigma senses through stigma testing. Moreover, participants seemed to find that the appearance of design looks like an elegant decoration. It also revealed that the user eating habits influence their preferences in choosing eating tools. 


\section{Limitation of research}

In the analysis stage of current AT utensils, there was a limitation of selected categories of utensils due to funds and time. The contrastive experiment (Creswell, 2007) aims to form a systematic evaluation about how AT cutlery sets can specifically help stroke patients. There are a considerable number of AT utensils that should be included in the quantity collection as well. In Torrens's (2001) study, there was a comparing experiment of seven different utensil product samples and evaluation about their usability. A recommendation of specific categories in addressing stroke AT cutlery usability should be conducted in the future.

The background research of the user target group would provide insights for this study, as well as the feedback from clinicians. However, the dining activity for anyone is a private routine to a certain extent. Considering about respecting the personal habits of patients, some investigations in this research would not be beyond an unexpected scope. For example, it is a challenge to stay with patients a whole day in order to achieve the goal of their eating preference and eating tools preference.

This study based its findings on the collection of qualitative data and produced findings affected by some subjective factors. The inherent bias from the researcher and subjective perspectives were involved in revising Vaes' (2014) PAMS question model. During the stigma user testing, the selected five participants only represented a small portion of the user group. In fact, a range of table manners and eating habits should be analyzed when addressing a design scenario of customization for stroke patients. 
When 3D printing was put into use in the prototyping process, it also highlighted several problems within the manufacturing process. The constraint that came about during this research was that the front portions of iterative prototypes were not guaranteed to work due to the material qualities and solutions. In the process of researching knife edges and spoon bowl angle, a significant impact on the printing qualities has affected the research process. 


\section{Future Work}

A significant future work is that the research proposes to undertake the user testing with recruited stroke patients who meet the inclusion criteria. In addition to future usability testing by stroke patients, the user stigma emotion should equally be addressed.

Further studies are required to establish a feasible customization service scenario for patients who have different impairments in their upper limbs. With the rapid technology developing in more product manufacturing, the development of AT products will reach a better achievement through technology intervention.

It is also important to remember the background research shows that cutting food is the most difficult aspect of eating. An effective and customized knife edge should primarily focus on patients' individual weaknesses. As stated in the earlier background research, the assistive devices are significantly influenced by the stroke patient's rehabilitation progress. Individual rehabilitation results decide the various conditions they may achieve, and one assumption for this limitation is that some stroke patients do not need AT cutlery for long-term use.

Although the early research process was guided by stroke professionals within the medical community, the perspective from patients is equally important to address. In addition to future usability testing by stroke patients, user stigma emotions should equally be addressed. 
130 



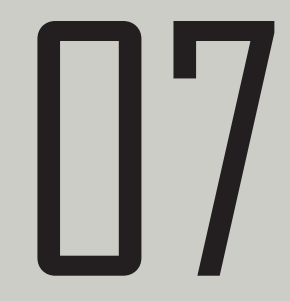

CDNCLUSION 
Strokes produce a range of impairments and survivors' adaptation styles are influential features of life after a stroke (Perry \& McLaren, 2002). The breadth encompassed by this research includes physical, emotional, psychological and social influences. The importance of AT devices and eating within the wider experience of a stroke was evident. This research has added to understanding of the experience post-stroke, linked with eating difficulties and developing the utensils. The important considerations have been highlighted for further research and interventions.

The aim of this dissertation was to address a stigma-free cutlery set and helping stroke patients with their hand difficulties. A predominant challenge this research revealed was moving the implements into a realm where they are no longer just functional and stigmatised utensils, but rather to enhance the intimate dining experience on both a perceptual and physical level. This research challenges the stereotypical idea of adaptive cutlery as an 'assistive device' through the streamlining of design and using processes. Applying research surrounding the perception of stroke and upper limb difficulty, the research presents a framework to formulate a new design language for adaptive implements. The output of this research has presented a complete cutlery set that provides users with comfortable and user pleasing culinary implements which they can use either at home or in public.

Reviews with clinicians and feedback from stigma testing helped substantiate and build on the functional and contextual requirements. It does help to better understand how stigma-free cutlery can help stroke patients' eating. Although the findings were from a subjective perspective, the design criteria and clinicians' feedback helped minimize biases and assumptions. 
The design requirements of the cutlery proved to be diverse and complex. Defining the functional criteria of the cutlery was majorly influenced by the patient's affected upper limb conditions. The physical aspects of the cutlery set have demonstrated that there is no one cutlery set that can cater to all categories of post-stroke users. This was shown in the discussion session with AUT clinicians, who discussed various types of impairments. Thus, a systematic map of cutlery categories should be developed for answering each upper limb difficulty in users. In contrast to some reports in the literature, there were few differences between Torrens's study (2013) and Philp's study (2001). These two studies have shown a number of cutlery sets were primarily designed for a commercial purpose and in mass production. Based on the initial findings, the development of a customizable design has shown a promising opportunity that enables users to have personalized cutlery by addressing their own difficulties.

Although this study has been tested with the bystander's perception and cultural factors, the main role in this research is still those people who have had a stroke. The user, bystanders, and culture are the compulsory components in addressing product-related stigma which contributed to this research. Without testing on stroke patients, the findings of this study were perfunctorily based on the expert clinicians' feedback and background research while there are a number of design criteria to form a conceptual framework. When presenting this conceptual framework, the intention is not able to answer all the questions that appear through the study. But, in the spirit of design, further research will provide compelling reference points for future developments. 



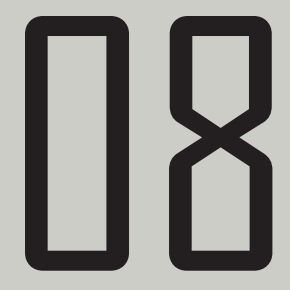

REFFERENCES 
Axelsson, A,N,K., Asplund, K., \& Norberg, A. (1984). Eating after acute stroke: towards an integrated view. International Journal of Nursing Studies 21(2):9399.

Axelsson, A,N,K., Asplund, K., Norberg, A., \& Alafuzoff I. (1998). Nutritional status in patients with acute stroke. ActaMedica Scandinavica 224:217-224.

Axelsson, A,N,K., Asplund, K., Norberg, A.,\& Ericsson, S. (1989). Eating problems and nutritional status during hospital stay of patients with severe stroke. Journal of the American Dietetic Association 8:4192-1096.

Anderson, R. (1992). The aftermath of stroke: The experience of patients and their families. Cambridge, United Kingdom: Cambridge University Press, pp. 150-154.

Axelsson, K., Asplund, K., Norberg, A., \& Eriksson, S. (1989). Eating problems and nutritional status during hospital stay of patients with severe stroke. Journal of the American Dietetic Association, 89(8), 1092-1096.

Anderson, R. (1992). The aftermath of stroke: The experience of patients and their families. Cambridge, United Kingdom: Cambridge University Press, pp. 150-154.

Andriga, A., Port, I. v., \& Meijer, J.-W. (2013). Long-Term Use of a Static HandWrist Orthosis in Chronic Stroke Patients: A Pilot Study. Opgeroepen op September 9, 2014, van Hindawi Publishing Organization: http://www. hindawi. com/journals/srt/2013/546093/

Barer, D. (1989). The natural history and functional consequences of dysphagia after hemispheric stroke. Journal of Neurology, Neurosurgery and Psychiatry, $52,236-241$.

Bevan, N., Kirakowski, J., \& Maissel, J. (1991). What is Usability? In H.-J. Bullinger (Ed.), Human aspects in computing: Design and use of interactive systems and work with terminals. Amsterdam, The Netherlands: Elsevier.

Becker, G. (1993). Continuity after a stroke: Implications of life course disruption in old age. Gerontology, 33(2), 148-158. 
Barer, D. (1989). The natural history and functional consequences of dysphagia after hemispheric stroke. Journal of Neurology, Neurosurgery and Psychiatry 52:236-241.

Burdick, A. (2003). Design (as) research. In B. Laurel (Ed.), Design research: Methods and perspectives (82). Cambridge, MA: MIT Press.

Brooke,J. (n.d.). SUS - A quick and dirty usability scale. Redhatch Consulting Ltd., 12 Beaconsfield Way, Earley, READING RG6 2UX United Kingdom

Carr, E. K., \& Hawthorn, P. J. (1988). Observation of eating skills after stroke: dribbling food and chewing. Clinical Rehabilitation, 2, 183-189.

Clarkson, J., et al. (2003). Inclusive design: Design for the whole population. Springer, pp.608.

Charllies, Z. (2015). Designed Prosthetics : a 120 thesis submitted to the Victoria University of Wellington in fulfilment of the requirements for the degree of Masters of Design Innovation. Victoria University of Wellington

Creswell J. W., \& Plano Clark V. (2007). Designing and Conducting Mixed Methods Research. London, United Kingdom: Sage.

Creswell, J. W. (2013). Research design: Qualitative, quantitative, and mixed methods approaches. Thousand Oaks, CA: Sage Publications.

Charllies, Z. (2015). Designed Prosthetics : a 120 thesis submitted to the Victoria University of Wellington in fulfilment of the requirements for the degree of Masters of Design Innovation. Victoria University of Wellington

Crandall, C. S. (2000). Ideology and lay theories of stigma: The justification of stigma. In T. F. Heatherton, R. E. Kleck, M. R. Hebl, \& J. G. Hull (Eds.), The social psychology of stigma (pp. 126-150). New York: Guilford.

Complete care shop. (n.d.). Eating Aids. 2016, June 13. Retrieved from https://www.completecareshop.co.uk/eating-aids/ 
Duncan,W. (2016). A game controller for stroke rehabilitation. Victoria University of Wellington.

Evil, J. (2013). Retrived from http://www.evilldesign.com/cortex

GSA Government-wide Section508 Accessibility Program (n.d.) Assistive Technology Act 1998. Retrieved from https://section508.gov/assistive-technology-act-1998.

Gariballa, S. E., Parker, S. G., \& Castleden, C. M. (1997). Nutritional status after stroke. Stroke, 108, 70.

Gibson, I., Rosen, D. W., Stucker, B. (2010). Additive manufacturing technologies. Springer-Verlag New York.

Garland-Thomson, R. (2016). Becoming Disabled. Retrieved from https://www. nytimes.com/2016/08/21/opinion/sunday/becoming-disabled.html.

Gill, C. J., Mukherjee, S. . S., Garland-Thomson, R., \& Mukherjee, D. (2016). Disability Stigma in Rehabilitation. PM and R, 8(10), 997-1003. DOI: 10.1016/j.pmrj.2016.08.028.

Huebner, M.L. (2002). The history of Cutlery. (n.p.).

Jacobsson, C., Axelsson, K., Osterlind, P. O., \& Norberg, A. (2000). How people with stroke and healthy older people experience the eating process. Journal of Clinical Nursing, 9 (2), 255-264.

Jacobson, S. (2014). Personalised assistive products: Managing stigma and expressing the self. Helsinki, Finland: Unigrafia.

Jacobson, S. (2010). Overcoming the Stigma Associated with Assistive Devices; Proceedings of the 7th international conference on Design \& Emotion, Spertus Institute, Chicago, US 
Jordan, P.W. (1996), Displeasure and how to avoid it. In S. Robertson (ed.), Contemporary Ergonomics 1996: Proceedings of the Annual Conference of the Ergonomics Society (University of Leicester, 10-12 April 1996), 56-61.

Kirchhof, P. D., Adamou, D. A., Knight, E., Lip, Y. H., Norrving, P. B., \& Pouvourville, P. G., et al. (2009). How can we avoid a stroke crisis? Retrieved from vanEscardio:http://www.escardio.org/communities/EHRA/publications/papers-interest/Documents/ehra-stroke-report-recommend-document.pdf

Kirakowski, J., \& Corbett, M. (1988), Measuring user aatisfaction. In D. M. Jones, \& R. Winder (Eds.), People and Computers IV. Cambridge, United Kingdom: Cambridge University Press.

Krippendorff, K.; Butter, R. (1984), Product semantics: Exploring the symbolic qualities of form, in Innovation: The Journal of the Industrial Designers Society of America (McLean, Va.: The Society), 3 (2), 4-9.

Kuniavsky, M. (2003). Observing the user experience. Burlington, US: Morgan Kaufmann.

Kuniavsky, M., Goodman, E., \& Moed, A. (2012). Observing the user experience: A practitioner's guide to user research (2nd ed). Amsterdam, The Natherlands; Boston , MA;'

Lincoln, N. B., \& Tinson, D. J. (1989). Relationship between subjective and objective memory impairment after stroke. British Journal of Clinical Psychology, 28, 61-65.

Lyden, P., \& Lau, G. T. (1991). A critical appraisal of stroke evaluation and rating scales. Stroke, 22, 1345-1352.

McLaren, S. M. G., Dickerson, J. W. T. (2000). Measurement of eating disability in an acute stroke population. Clinical Effectiveness in Nursing, 4, 109-120.

McLaren, S. M. G., Dickerson, J. W. T. (2000). Measurement of eating disability in an acute stroke population. Clinical Effectiveness in Nursing, 4, 109-120. 
National Stroke Association. (2014). What is stroke? Opgeroepen op September 10, 2014, van National Stroke Association: http://www.stroke.org/site/ PageServer?pagename=stroke.

Martin, B., \& Hanington, B. M. (2012). Universal methods of design: 100 ways to research complex problems, develop innovative ideas, and design effective solutions. Beverly, MA: Rockport Publishers.

McDonagh-Philp, D. C., Lebbon, C., \& Torren, G.E. (1999). An evidence based design method within a user-centred design approach. In Proceedings of The 4th Asian Design Conference International Symposium on Design Science. Nagaoka, Japan: The Program Committee. [CD-ROM].

Marzano, RJ. (1998). A Theory-Based Meta-Analysis of Research on Instruction. Mid-continent Regional Educational Laboratory, Aurora, Colorado.

Norman, D. A. (2002). Emotion and design: Attractive things work better. Interactions Magazine, 4, 36-42.

Norman, D. (2004), Emotional Design: Why We Love (or Hate) Everyday Things, New York: Basic Books.

Phillips, B. M. S., \& Zhao H. (1993). Predictors of Assistive Technology Abandonment. Assistive Technology, 5, 36-451.

Pullin,G. (2009). Design meets disability. Cambridge, MA: MIT Press.

Perry, L., \& McLaren, S. (2002). Coping and adaptation at six months after stroke: experiences with eating disabilities. International Journal of Nursing Studies, 40(2003) 185-195.

Perry, L. (2004). Eating and dietary intake in communication-impaired stroke survivors: a cohort study from acute-stage hospital admission to 6 months poststroke. Health Care Research Unit, City University, 20, Bartholomew Close, London, EC1A 7QN, USA 
Phillips B., M.S. \& Zhao H. (1993). Predictors of Assistive Technology Abandonment, Assistive Technology, Vol 5, pp.36-451, 1993.

Rubin, J., \& Chisnell, D. (2011). Handbook of usability testing: How to plan, design, and conduct effective tests. Indianapolis, IN: Wiley.

Rehabmart. (n.d.). Adaptive Eating Utensils, Weighted Silverware, Swivel Spoon, Special Needs Utensils. 2016. June 13. Retrieved from http://www.rehabmart.com/product/comfort-grip-utensils-6522.html

Renda, G., Jackson, S., Kuys, B., \& Allan Whitfield, T. W. (2016). The cutlery effect: do designed products for people with disabilities stigmatise them?, Disability and Rehabilitation: Assistive Technology, 11:8, 661-667, DOI: 10.3109/17483107.2015.1042077

Shirzad, N., Valdés, B. A., Hung, C.-T., Law, M., Hay, J., \& Van der Loos, H. F. (2015). FEATHERS, a bimanual upper limb rehabilitation platform: A case study of user-centred approach in rehabilitation device design. University of British Columbia. Retrieved from https://open.library.ubc.ca/clRcle/collections/42591/items/1.0228382

Skogsr $\varnothing d, I, \varnothing$. (n.d). Empathy and Aesthetics: Combating Stigma in the Design of Assistive Products. (n.p.).

Stroke Association. (2013). Physical effects of stroke. Opgeroepen op September 9, 2014, van Stroke Association.

S. Nijenhuis, M. (2014, September 22). Hoe ziet de therapie voor CVA patiënten eruit? (E. v. Berg, Interviewer)

Stehle,C. \& Albrecht-Buehler, C. (2008). Developing More Desirable Products for Stroke Survivors, Topics in Stroke rehabilitation, 15:2,109-117,DOI: 10.1310/tsr1502-109

Thibaut, A., Chatelle,C., Ziegler, E., Bruno, M., Laureys, S. \& Gosseries, O. (2013). Spasticity after stroke: Physiology, assessment and treatment. Informa healthcare. 
Torrens, G. E., \& Smith, N. C. S. (2013). Evaluation of an assistive technology product design using a paired comparisons method within a mixed methods approach: A case study evaluating preferences for four types of cutlery with 34 upper limb impaired participants. Disability and Rehabilitation: Assistive Technology, 8(4), 340-34.

Torrens G., McDonagh-Philp, D., \& Newman A. (2001). Getting a grip. Ergonomics in Design: The Quarterly of Human Factors Applications, 9, 7-13.

Tran,T., \& Park, J. (2015). Development of a Framework to Customize Design Methodologies for Product Service Systems. Proceedings of the 2015 International Conference on Industrial Engineering and Operations Management. Dubai, United Arab Emirates.

Vaes, K., Stappers, P. J., Standaert, A., \& Desager,K. (2012). Contending stigma in product design: Using insights from social psychology as a stepping stone for design strategies. Proceedings of the International Design and Emotion Conference. London, United Kingdom.

Vaes K. (2014). Product Stigmaticity - Understanding, Measuring and Managing Product- Related Stigma. Delft University of Technology - Antwerp University.

Wade, D.T., (1994). Stroke. In: Stevens, A., Raftery, J. (Eds.), Health Care Needs Assessment, Vol. 1. Radcliffe Medical Press, Oxford, p. 179.

Wolfe, C.D., (2000). The impact of stroke. British Medical Bulletin 56 (2), 275-286.

World Health Organisation. (2009). International Classification of Functioning, Disability and Health (ICF). Geneva, WHO Press.

Wood, L. E. (1997). Semi-structured interviewing for user-centered design. Interactions, 4(2), 48-61.

Wilkinson, C. R., \& Antonella De Angeli, A. (2014). Applying user centered and participatory design approaches to commercial product development. Design Studies, 35(6), 614-631. 
Wellington mobility centre. (n.d.). Cutlery \& Utensils. 2016. June 13. Retrieved fromhttps://www.activemobility.co.uk/cutlery-kitchen-utensils-c223_53_54/ index.html

Young, R. R. (1994). Spasticity: A review. Neurology ;44(S9):S12-S20.

Zimmerman, J., Forlizzi, J., \& Evenson, S. (2007). Research through design as a method for interaction design research in $\mathrm{HCl}$. In Proceedings of the SIGCHI conference on human factors in computing systems (pp. 493-502). ACM. Retrieved from http://dl.acm.org/citation.cfm?id=1240704. 



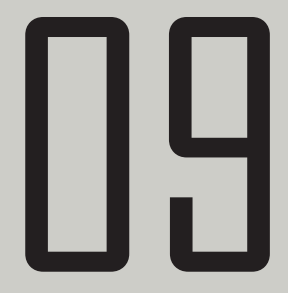

FISURE LIST 
Figure 1.0 Hand patterns

BOTOX. (2017).THE SIGNS OF SPASTICITY. Retrieved from https://www.botoxspasticity.com/about-spasticity/how-diagnosed/

\section{Figure 1.2 Collection of Historical Cutlery}

Insert image of cutlery adopted from

Peter, B. (2001). British cutlery : an illustrated history of its design, evolution and use. London : Philip Wilson.

Figure 1.8, $1.9 \& 1.10$ Original the product appraisal model for stigma

Figure 2.4 The Product intervention for stigma

Figure 3.5 Evaluate model of product stigma

Vaes K. (2014). Product Stigmaticity - Understanding, Measuring and Managing

Product-Related Stigma. Delft University of Technology - Antwerp University.

Figure 1.20 3D print cast: Cortex

Evill, J. (2013). Exoskeleton protecting the internal skeleton. Retrieved from http://www.evilldesign.com/cortex

Figure 1.21 3D Metal print cutlery Eragatory. (2017). 3D PRINTED 18 KT GOLD PLATED CUTLERY SET. Retrieved from http://eragatory.blogspot.co.nz/2013/06/3d-printed-18-kt-gold-plated-cutleryset.html

Figure 1.22 3D stainless steel cutlery Laboratory for Explorative Architecture\& Design. (2017). Jewel Silverware Cutelry Set. Reterieved from http://I-e-a-d.pro/portfolio/jewel-silverware-3/

\section{Figure 3.2 Summary of upper limb pattern}

Thibaut, A., Chatelle,C., Ziegler, E., Bruno, M., Laureys, S. \& Gosseries, O. (2013). Spasticity after stroke: Physiology, assessment and treatment. Informa healthcare. 
All others figures, tables, diagrams and renderings made by author. 



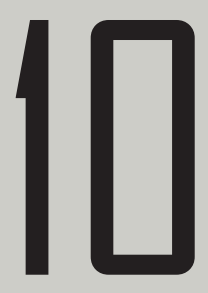

APPENDIX 


\section{Designing a System for Stroke Rehabilitation INFORMATION SHEET FOR PARTICIPANTS}

Thank you for your interest in this project. Please read this information before deciding whether or not to take part. If you decide to participate, thank you. If you decide not to take part, thank you for considering my request.

\section{Who am I?}

My name is Lin Chen and I am a Masters student in the School of Design at Victoria University of Wellington. This research project is work towards my thesis.

\section{What is the aim of the project?}

This project aims to design a set of cutlery for stroke patient, and help stroke patients carry out their daily diet. This research has been approved by the Victoria University of Wellington Human Ethics Committee [23011].

\section{How can you help?}

If you agree to take part I will interview you in your office, a meeting room in the School of Design's campus or in a public place, such as a café. I will ask you questions about stroke rehabilitation. I will audio record the interview and write it up later. We will construct a set of criteria and designs that facilitate stroke rehabilitation based on the findings from the research. In a second interview, we will seek your feedback about the new designs. Each interview will take 60 minutes. You can stop the interviews at any time, without giving a reason. You can withdraw from the study up to four weeks after the first interview. After this time, we will use the information you provide to design new objects. You can also withdraw your information for the second interview up to four weeks after it occurs. If you withdraw, the information you provided will be destroyed or returned to you.

\section{What will happen to the information you give?}

This research is confidential. I will not name you in any reports, and I will not include any information that would identify you. Only my supervisors and I will read the notes or transcript of the interview. The interview transcripts, summaries and any recordings will be kept securely and destroyed 3 years after the research ends.

\section{What will the project produce?}

The information from my research will be used in my Master's thesis. You will not be identified in my report. I may also use the results of my research for conference presentations, and academic reports. I will take care not to identify you in any presentation or report. 


\section{If you accept this invitation, what are your rights as a research participant?}

You do not have to accept this invitation if you don't want to. If you do decide to participate, you have the right to:

- choose not to answer any question;

- $\quad$ ask for the recorder to be turned off at any time during the interview;

- $\quad$ withdraw from the study up until four weeks after your interview;

- $\quad$ ask any questions about the study at any time;

- $\quad$ receive a copy of your interview recording (if it is recorded);

- $\quad$ read over and comment on a written summary of your interview;

- $\quad$ agree on another name for me to use rather than your real name;

- be able to read any reports of this research by emailing the researcher to request a copy.

\section{If you have any questions or problems, who can you contact?}

If you have any questions, either now or in the future, please feel free to contact either:

\section{Student:}

Name: Lin Chen

University email address:

Shuilidieyi@hotmail.com

chenlin@myvuw.ac.nz

\section{Supervisor:}

Name: Dr Edgar Rodriguez

Role: Programme Director Industrial Design

School: School of Design

Phone: 045636544

edgar.rodriguez@vuw.ac.nz

\section{Human Ethics Committee information}

If you have any concerns about the ethical conduct of the research you may contact the Victoria University HEC Convener: Associate Professor Susan Corbett. Email susan.corbett@vuw.ac.nz or telephone +64-4-463 5480. 


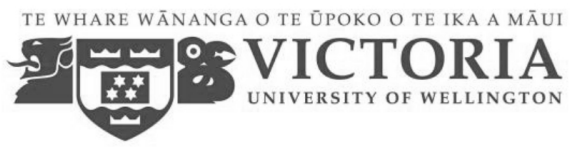

\section{Designing a cutlery set for Stroke Patient CONSENT TO INTERVIEW}

Researcher: Lin Chen, School of Design, Victoria University of Wellington.

- I have read the Information Sheet and the project has been explained to me. My questions have been answered to my satisfaction. I understand that I can ask further questions at any time.

- I agree to take part in an audio recorded interview.

I understand that:

- I may withdraw from this study up to four weeks after the first interview or up to four weeks after the second interview reviewing the designs, and any information that I have provided will be returned to me or destroyed.

- $\quad$ The information I have provided will be destroyed 5 years after the research is finished.

- $\quad$ Any information I provide will be kept confidential to the researcher and the supervisor. I understand that the results will be used for a Masters/PhD report and a summary of the results may be used in academic reports and/or presented at conferences.

- $\quad$ My name will not be used in reports, nor will any information that would identify me.

- I would like a summary of my interview:

Yes No $\mathrm{O}$ would like to receive a copy of the final report and have added my Yes I would like to receive
email address below.

Yes No $O$

Signature of participant:

Name of participant:

Date:

Contact details: 
Committees

Aitken Street

PO Box 5013

21 April 2016

Dr Brian Robinson

Graduate School of Nursing, Midwifery \& Health

PO Box 7625

Newtown 6242

Dear Dr Robinson

$\begin{array}{lll}\mathrm{Re}: & \text { Ethics ref: } & 16 / \text { CEN/5 } \\ & \text { Study title: } & \text { Developing Interactive Devices and Games for Physical Therapies in } \\ & \text { Stroke Recovery }\end{array}$

I am pleased to advise that this application has been approved by the Central Health and Disability Ethics Committee. This decision was made through the HDEC-Full Review pathway.

\section{Conditions of HDEC approval}

HDEC approval for this study is subject to the following conditions being met prior to the commencement of the study in New Zealand. It is your responsibility, and that of the study's sponsor, to ensure that these conditions are met. No further review by the Central Health and Disability Ethics Committee is required.

Standard conditions:

1. Before the study commences at any locality in New Zealand, all relevant regulatory approvals must be obtained.

2. Before the study commences at any locality in New Zealand, it must be registered in a clinical trials registry. This should be a WHO-approved (such as the Australia New Zealand Clinical Trials Registry, www.anzctr.org.au). However https://clinicaltrials.gov/ is acceptable provided registration occurs prior to the study commencing at any locality in New Zealand.

3. Before the study commences at a given locality in New Zealand, it must be authorised by that locality in Online Forms. Locality authorisation confirms that the locality is suitable for the safe and effective conduct of the study, and that local research governance issues have been addressed.

Non-standard conditions:

- The Participant Information Sheet (PIS) was not tracked, making it difficult to check. Please ensure future submissions are supported with tracked versions of documents. 
- The section in the PIS headed, "What are the possible benefits and risks of this study" still does not include benefits and risks to participants therefore this section's title should be changed.

Non-standard conditions must be completed before commencing your study. Nonstandard conditions do not need to be submitted to or reviewed by HDEC before commencing your study.

If you would like an acknowledgement of completion of your non-standard conditions letter you may submit a post approval form amendment. Please clearly identify in the amendment that the changes relate to non-standard conditions and ensure that supporting documents (if requested) are tracked/highlighted with changes.

For information on non-standard conditions please see section 128 and 129 of the Standard Operating Procedures at http://ethics.health.govt.nz/home.

\section{After HDEC review}

Please refer to the Standard Operating Procedures for Health and Disability Ethics Committees (available on www.ethics. health.govt.nz) for HDEC requirements relating to amendments and other post-approval processes.

Your next progress report is due by 20 April 2017.

\section{Participant access to ACC}

The Central Health and Disability Ethics Committee is satisfied that your study is not a clinical trial that is to be conducted principally for the benefit of the manufacturer or distributor of the medicine or item being trialled. Participants injured as a result of treatment received as part of your study may therefore be eligible for publicly-funded compensation through the Accident Compensation Corporation (ACC).

Please don't hesitate to contact the HDEC secretariat for further information. We wish you all the best for your study.

Yours sincerely,

Mrs Helen Walker

Chairperson

Central Health and Disability Ethics Committee

Encl: appendix A: documents submitted appendix $B$ : statement of compliance and list of members 


\section{Appendix A}

Documents submitted

\begin{tabular}{|l|l|l|}
\hline Document & Version & Date \\
\hline $\begin{array}{l}\text { Covering Letter: New Covering Letter indicating to responses and } \\
\text { changes made }\end{array}$ & 1 & 05 April 2016 \\
\hline CV for Cl: CI CV & 1 & 11 December 2015 \\
\hline Evidence of scientific review: Peer Review & 1 & 11 December 2015 \\
\hline Protocol: Updated Protocol for Research & 2 & 04 April 2016 \\
\hline $\begin{array}{l}\text { Survey/questionnaire: Demographic questions and examples of } \\
\text { questions for semi-structured interviews and recruitment information }\end{array}$ & 1 & 11 December 2015 \\
\hline $\begin{array}{l}\text { PIS/CF: Example of Participant Information Sheet and Consent } \\
\text { Form }\end{array}$ & 1 & 05 April 2016 \\
\hline Application & & \\
\hline Response to Request for Further Information & & 06 April 2016 \\
\hline
\end{tabular}




\section{Appendix B}

Statement of compliance and list of members

\section{Statement of compliance}

The Central Health and Disability Ethics Committee:

- is constituted in accordance with its Terms of Reference

- operates in accordance with the Standard Operating Procedures for Health and Disability Ethics Committees, and with the principles of international good clinical practice (GCP)

- is approved by the Health Research Council of New Zealand's Ethics Committee for the purposes of section 25(1)(c) of the Health Research Council Act 1990

— is registered (number 00008712) with the US Department of Health and Human Services' Office for Human Research Protection (OHRP).

\section{List of members}

\begin{tabular}{|l|l|l|l|}
\hline Name & Category & Appointed & Term Expires \\
\hline Mrs Helen Walker & $\begin{array}{l}\text { Lay (consumer/community } \\
\text { perspectives) }\end{array}$ & $01 / 07 / 2015$ & $01 / 07 / 2018$ \\
\hline Dr Angela Ballantyne & Lay (ethical/moral reasoning) & $30 / 07 / 2015$ & $30 / 07 / 2018$ \\
\hline Dr Melissa Cragg & Non-lay (observational studies) & $30 / 07 / 2015$ & $30 / 07 / 2018$ \\
\hline Dr Peter Gallagher & $\begin{array}{l}\text { Non-lay (health/disability service } \\
\text { provision) }\end{array}$ & $30 / 07 / 2015$ & $30 / 07 / 2018$ \\
\hline Mrs Sandy Gill & $\begin{array}{l}\text { Lay (consumer/community } \\
\text { perspectives) }\end{array}$ & $30 / 07 / 2015$ & $30 / 07 / 2018$ \\
\hline Dr Patries Herst & Non-lay (intervention studies) & $27 / 10 / 2015$ & $27 / 10 / 2018$ \\
\hline Dr Dean Quinn & Non-lay (intervention studies) & $27 / 10 / 2015$ & $27 / 10 / 2018$ \\
\hline Dr Cordelia Thomas & Lay (ethical/moral reasoning) & $19 / 05 / 2014$ & $19 / 05 / 2017$ \\
\hline
\end{tabular}

Unless members resign, vacate or are removed from their office, every member of HDEC shall continue in office until their successor comes into office (HDEC Terms of Reference)

\section{http://www.ethics.health.govt.nz}




\title{
Participant Information Sheet
}

\author{
Study title: $\quad$ A Set of Cutlery for Stroke Survivors \\ $\begin{array}{lll}\text { Locality: } & \text { Wellington } & \text { Ethics committee ref.: }\end{array}$ \\ $\begin{array}{llll}\text { Lead investigator: } & \text { Brian Robinson } & \text { Contact phone number: } & \text { (04) } 4636155\end{array}$
}

You are invited to take part in a study on the design of a set of cutlery for stroke survivors. Whether or not you take part is your choice. If you don't want to take part, you don't have to give a reason, and it won't affect the care you receive. If you do want to take part now, but change your mind later, you can pull out of the study at any time.

This Participant Information Sheet will help you decide if you'd like to take part. It sets out why we are doing the study, what your participation would involve, what the benefits and risks to you might be, and what would happen after the study ends. We will go through this information with you and answer any questions you may have. You do not have to decide today whether or not you will participate in this study. Before you decide you may want to talk about the study with other people, such as family, whānau, friends, or healthcare providers. Feel free to do this.

If you agree to take part in this study, you will be asked to sign the Consent Form on the last page of this document. You will be given a copy of both the Participant Information Sheet and the Consent Form to keep.

This document is 6 pages long, including the Consent Form. Please make sure you have read and understood all the pages.

\section{WHAT IS THE PURPOSE OF THE STUDY?}

This study is to develop a set of cutlery that can be used by people who are recovering from stroke.

We are wanting to know how you find using the new set of cutlery. Our aim is that these will be easy to use and understand.

This set of cutlery is developed by students as a requirement for a Masters degree. This research is funded by the School of Design at Victoria University of Wellington.

Any other questions you have can be answered by Dr. Brian Robinson (463 6155)

This research has been approved by the Health and Disability Ethics Committee. 
WHAT WILL MY PARTICIPATION IN THE STUDY INVOLVE?

We asked you to take part in this research because you have had a stroke in the past 12 months and may have limited use of one of your legs or arms.

The research study will take place either at a Stroke Club or in your home.

If the research study takes place in your home, a research student will come. They will bring mobile telephones with them so that they can contact their research supervisors.

We will ask some questions about you such as how old you are, your ethnic background, how long ago you had the stroke and how the stroke affects you now.

We will show you a set of cutlery.

You will be asked to use the set of cutlery.

You can use it for as long as you like and can tell us when you want to stop.

We will take a video and photographs of you using this set of cutlery. This is to make sure that using the set $f$ cutlery will be useful and not cause harm. Stroke rehabilitation physiotherapists will review these recordings. We will keep the video and photographs securely in the University. Because other researchers will be interested in our research we may show the photographs or a video of you. Your involvement in the study will only be known by the researchers. All photographs and videos will be taken using cameras belonging to the School of Design. The images and videos will be taken off these cameras and immediately after this session and then kept secure in the University computer system.

If we do use photographs or videos of you for presenting our research we will not show any part of you, such as your face, that can tell other people that you have taken part. If we take pictures in your home, we will also make sure that we do not show anything that identifies your house or that you took part. We will do this by blurring parts of the images and videos.

We will ask you for your thoughts on using the computer control and game. We will record what you say. If you tell us something useful that we quote, we will not use your name with what you say.

Your participation requires your concentration using the set of cutlery. We realize that this can be tiring for you so we ask you can tell that you are wanting to rest or to stop the session. You may be invited to take part again if you would like to help us test changes.

\section{WHAT ARE THE POSSIBLE BENEFITS AND RISKS OF THIS STUDY?}

We know that people who have had stroke cannot access stroke rehabilitation therapy regularly. They have to travel to clinics or hospital. We also know that rehabilitation is more effective when it is carried out for several hours throughout the day, every day.

This study is to support people who have had a stroke to provide stroke rehabilitation therapy in their home. This can be by themselves or with the help of carer support or family members.

We are wanting to find out whether this set of cutlery may be useful in stroke rehabilitation. This research is finding out whether you can use it and what you think of it.

This does not replace any other therapy you may be receiving. We are not using the set of cutlery as part of your therapy at this stage. We want to find out whether this might be usable for stroke survivors.

While you are using the set of cutlery you will be sitting in a chair. We will want you to stay sitting. 
WHO PAYS FOR THE STUDY?

This study is funded by Victoria University of Wellington and the School of Design through medical technology research grants from the Centre of Research Excellence of Medical Technologies.

You will not incur any costs by taking part and we will travel to you.

\section{WHAT IF SOMETHING GOES WRONG?}

If you were injured in this study, which is unlikely, you would be eligible for compensation from ACC just as you would be if you were injured in an accident at work or at home. You will have to lodge a claim with ACC, which may take some time to assess. If your claim is accepted, you will receive funding to assist in your recovery.

\section{WHAT ARE MY RIGHTS?}

You are volunteering to take part. You do not have to take part in this study and you can withdraw at anytime.

We can show you the video recording and photographs of you we have collected. We can also give you a copy of what we have recorded you saying to us about using the computer device and game.

It is unlikely that participating will affect your health but if it does, we will contact you immediately.

We will not identify you in any of the students work or presentations of the work.

\section{WHAT HAPPENS AFTER THE STUDY OR IF I CHANGE MY MIND?}

After you have taken part and change your mind about being involved, please contact the researcher (the design student) or the lead investigators (Brian Robinson, in the first instance, or Edgar Rodriguez) and any data, information and images associated with your participation will be destroyed.

We will securely store the information and data you have provided for five (5) years and it will then be destroyed.

We can present the findings of this study at stroke clubs within a year of conducting the study.

We can also send you a summary of the student's thesis describing the outcome of the study.

We may also present this study with other similar studies we are conducting at conferences or in books or journals. 


\section{WHO DO I CONTACT FOR MORE INFORMATION OR IF I HAVE CONCERNS?}

If you have any questions, concerns or complaints about the study at any stage, you can contact:

Dr Brian Robinson, Senior Lecturer, Graduate School of Nursing, Midwifery \& Health, Victoria University of Wellington.

Work phone: (04) 9349321

brian.robinson@vuw.ac.nz

24 Hour contact numbers:

Dr Robinson: 0297769321

If you cannot contact Dr Robinson, please contact

Associate Professor Edgar Rodriguez: 0275636544

If you have other questions, concerns or complaints and wish to contact a Māori support person, you can content.

Katherine Rel Lin Chen

ıry Group - Māori, CCDHB

Work phone: (04) 8062524

If you want to talk to someone who isn't involved with the study, you can contact an independent health and disability advocate on:

\begin{tabular}{|c|c|}
\hline Phone: & 0800555050 \\
\hline Fax: & 08002 SUPPORT (0800 2787 7678) \\
\hline Email: & advocacy@hdc.org.nz \\
\hline
\end{tabular}

For Maori health support please contact your health provider and they will refer you to the representative Maori health support group.

You can also contact the health and disability ethics committee (HDEC) that approved this study on:

Phone: $\quad 08004$ ETHICS

Email: hdecs@moh.govt.nz 


\section{Consent Form}

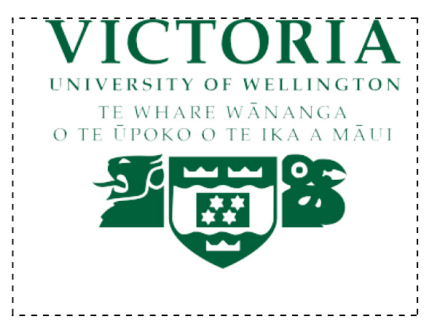

If you need an INTERPRETER, please tell us.

If you are unable to provide interpreters for the study, please clearly state this in the Participant Information Sheet

\section{Please tick to indicate you consent to the following}

I have read, or have had read to me in my first language, and I understand the Participant Information Sheet.

Yes $\square$

I have been given sufficient time to consider whether or not to participate in this study.

Yes $\square$

I have had the opportunity to use a legal representative, whanau/ family support or a friend to help me ask questions and understand the study.

I am satisfied with the answers I have been given regarding the study and I have a copy of this consent form and information sheet.

Yes $\square$

I understand that taking part in this study is voluntary (my choice) and that I may withdraw from the study at any time without this affecting my medical care.

Yes $\square$

I consent to the research staff collecting and processing my information, including information about my health.

Yes $\square$

I understand that my participation in this study is confidential and that no material, which could identify me personally, will be used in any reports on this study.

I consent to the research staff taking pictures or video recordings of me and I understand that if used in presentations, these will be altered so that I or my involvement cannot be identified.

Yes $\square$

I understand the compensation provisions in case of injury during the study.

Yes $\square$

I know who to contact if I have any questions about the study in general.

Yes $\square$

I understand my responsibilities as a study participant.

Yes $\square$

I wish to receive a summary of the results from the study.

Yes $\mathrm{C}$

No $\square$ 
Declaration by participant:

I hereby consent to take part in this study.

Participant's name:

Signature:

Date:

\section{Declaration by member of research team:}

I have given a verbal explanation of the research project to the participant, and have answered the participant's questions about it.

I believe that the participant understands the study and has given informed consent to participate.

Researcher's name: Lin Chen 

